\title{
Decarboxylative Radical Azidation using MPDOC and MMDOC esters
}

\author{
Erich Nyfeler and Philippe Renaud* \\ Department of Chemistry and Biochemistry \\ University of Berne \\ Freiestrasse 3 \\ CH-3012 Berne, Switzerland \\ philippe.renaud@ioc.unibe.ch
}

\section{Supporting Information}

General. Melting points: Büchi B-545 apparatus, uncorrected. ${ }^{1} \mathrm{H}-$ and ${ }^{13} \mathrm{C}-\mathrm{NMR}$ spectra: Bruker AVANCE-300 spectrometer operating at $300 \mathrm{MHz}$ for ${ }^{1} \mathrm{H}$ - and $75 \mathrm{MHz}$ for ${ }^{13} \mathrm{C}-\mathrm{NMR}$. Some ${ }^{1} \mathrm{H}$ - and ${ }^{13} \mathrm{C}$-NMR spectra were recorded on a Bruker DRX-400 spectrometer. Chemical shift data are reported in units of $\delta$ (ppm) using as the internal standard either residual $\mathrm{CHCl}_{3}$ ( $\delta=7.26$ for ${ }^{1} \mathrm{H}-\mathrm{NMR}$ spectra and $\delta=77.0$ for ${ }^{13} \mathrm{C}-\mathrm{NMR}$ spectra $)$ or $\mathrm{Si}\left(\mathrm{CH}_{3}\right)_{4}\left(\delta=0\right.$ for ${ }^{1} \mathrm{H}$ $\mathrm{NMR}$ and ${ }^{13} \mathrm{C}-\mathrm{NMR}$ spectra). Multiplicities are given as $\mathrm{s}$ (singlet), $\mathrm{d}$ (doublet), $\mathrm{t}$ (triplet), $\mathrm{q}$ (quartet), $\mathrm{m}$ (multiplet), and br (broad) for ${ }^{1} \mathrm{H}$ spectra. Infrared spectra: Jasco FT-IR-460 Plus Spectrometer equipped with a Specac MKII Golden Gate Single Reflection Diamond ATR System. LRMS and HRMS: Waters Micromass Autospec $Q$ mass spectrometer in EI mode at 70 eV. GC-MS: Finnigan Trace GC 2000 gas chromatograph equipped with an autosampler 
and a Trace MS mass selective detector. GC: CE instruments HRGC series 8532 using $M N$ optima $\delta$-3, ID $0.25 \mu \mathrm{M}, 30 \mathrm{~m}$.

TLC: Merck silica gel $60 \mathrm{~F}_{254}$ plates. Visualization of TLC plates was accomplished according to the following methods: $254 \mathrm{~nm}$ UV light; dipping in different staining solutions: $\mathrm{KMnO}_{4}(5 \mathrm{~g}), \mathrm{Na}_{2} \mathrm{CO}_{3}(30 \mathrm{~g})$ in $\mathrm{H}_{2} \mathrm{O}(500 \mathrm{~mL})$; phosphomolybdic acid (25 g), cerium sulfate $(10 \mathrm{~g})$ and $\mathrm{H}_{2} \mathrm{SO}_{4}$ conc. $(5 \mathrm{~mL})$ in $\mathrm{H}_{2} \mathrm{O}(940 \mathrm{~mL})$; ninhydrin $(1 \mathrm{~g})$ and acetic acid $(25 \mathrm{~mL})$ in tert-butanol $(225 \mathrm{~mL})$; vanilline $(3 \mathrm{~g})$ and $\mathrm{H}_{2} \mathrm{SO}_{4}$ conc $(4 \mathrm{~mL})$ in $\mathrm{EtOH}(250 \mathrm{~mL})$ and subsequent heating. Flash column chromatography (FC): $S d S$ Silica gel $60(40-63 \mu \mathrm{m})$. Commercial reagents were used as received. Solvents for reactions (THF, $\mathrm{Et}_{2} \mathrm{O}, \mathrm{CH}_{2} \mathrm{Cl}_{2}$, $\mathrm{CH}_{3} \mathrm{CN}$, benzene and toluene) were filtered over columns of activated alumina under a positive pressure of argon. Solvents for extractions and FC were of technical grade and distilled before use. Irradiation was performed using an Osram Ultra Vitalux $300 \mathrm{~W}$ sun lamp.

\section{General Procedure A: Preparation of acyl thiohydroxamate via the corresponding acyl}

chloride. The carboxylic acid $(4.0 \mathrm{mmol})$ was heated under reflux in $\mathrm{SOCl}_{2}(6 \mathrm{~mL}, 80 \mathrm{mmol})$ for $4 \mathrm{~h}$. The excess $\mathrm{SOCl}_{2}$ was completely removed by distillation. The residue was dissolved in $\mathrm{CH}_{2} \mathrm{Cl}_{2}(100 \mathrm{~mL})$ and thiohydroxamic acid $(4.4 \mathrm{mmol})$ was added. The reaction flask was protected from light with an aluminum foil and $\mathrm{Et}_{3} \mathrm{~N}(0.6 \mathrm{~mL}, 8.3 \mathrm{mmol})$ was added over a period of $30 \mathrm{~min}$ while maintaining the temperature at $0{ }^{\circ} \mathrm{C}$. The reaction mixture was stirred for $5 \mathrm{~h}$, diluted with more $\mathrm{CH}_{2} \mathrm{Cl}_{2}$ and washed with $\mathrm{H}_{2} \mathrm{O}$. The organic layer was dried over $\mathrm{MgSO}_{4}$ and concentrated. The crude product was purified by FC.

General Procedure B: Preparation of acyl thiohydroxamate with DCC. The carboxylic acid $(0.9 \mathrm{mmol})$ was added to a solution of DCC (210 $\mathrm{mg}, 1.0 \mathrm{mmol})$ and DMAP (12 $\mathrm{mg}, 0.1$ mmol) in $\mathrm{CH}_{2} \mathrm{Cl}_{2}(15 \mathrm{~mL})$ at $0{ }^{\circ} \mathrm{C}$ and the reaction mixture was stirred for $30 \mathrm{~min}$. The flask was covered with an aluminum foil and a solution of the thiohydroxamic acid $(0.9 \mathrm{mmol})$ in $\mathrm{CH}_{2} \mathrm{Cl}_{2}(5 \mathrm{~mL})$ was added slowly at $0{ }^{\circ} \mathrm{C}$. The mixture was stirred overnight at $\mathrm{rt}$, filtered though Celite ${ }^{\circledR}$ and concentrated. The residue was dissolved in $\mathrm{Et}_{2} \mathrm{O}$, filtered through Celite ${ }^{\circledR}$, concentrated and purified by FC to give the desired ester. 
General Procedure C: Rearrangement of acyl thiohydromates (MPDOC esters). The MPDOC ester $(0.6 \mathrm{mmol})$ was dissolved in $\mathrm{CH}_{2} \mathrm{Cl}_{2}(20 \mathrm{~mL})$ and irradiated with a sun lamp at $5{ }^{\circ} \mathrm{C}$ for $2 \mathrm{~h}$. Concentration and purification by $\mathrm{FC}$ afforded the rearranged product.

General Procedure D: Decarboxylative Azidation under photochemical initiation. The MPDOC ester $(1.4 \mathrm{mmol})$ in $\mathrm{CH}_{2} \mathrm{Cl}_{2}(10 \mathrm{~mL})$ was added over a period of $4 \mathrm{~h}$ with a syringe pump to an irradiated (300 W sun lamp) solution of benzenesulfonyl azide (1.5 g, $8.3 \mathrm{mmol})$ in $\mathrm{CH}_{2} \mathrm{Cl}_{2}(40 \mathrm{~mL})$ at $5{ }^{\circ} \mathrm{C}$. Concentration under vacuum and purification by $\mathrm{FC}$ gave the desired alkyl azide.

General Procedure E: Decarboxylative Azidation under thermal initiation using AIBN. The MMDOC or MPDOC ester $(0.6 \mathrm{mmol})$ in benzene $(10 \mathrm{~mL})$ was added with a syringe pump over a period of $4 \mathrm{~h}$ to a refluxing solution of benzenesulfonyl azide $(660 \mathrm{mg}, 3.6$ $\mathrm{mmol}$ ) and AIBN (49 mg, $0.3 \mathrm{mmol})$ in benzene. Additional portions of AIBN (49 mg, 0.3 mmol) were added every hour until complete conversion of the starting material. Concentration under reduced pressure and purification by FC gave the desired azide.

Methyl hydroxy(methyl)carbamodithioate. $\mathrm{Et}_{3} \mathrm{~N}(1.55 \mathrm{~mL}, 11.0 \mathrm{mmol})$ was added over a $\mathrm{HO}^{-\mathrm{N}} \prod_{\mathrm{S}}^{\mathrm{I}} \mathrm{S}$ period of $5 \mathrm{~min}$ to a mixture of $\mathrm{N}$-methyl hydroxylamine hydrochloride ( 835 $\mathrm{mg}, 10.0 \mathrm{mmol})$, carbon disulfide $(838 \mathrm{mg}, 11.0 \mathrm{mmol})$ and iodomethane ( $1.56 \mathrm{~g}, 11.0 \mathrm{mmol})$ in $20 \mathrm{~mL}$ of $\mathrm{CH}_{2} \mathrm{Cl}_{2}$ at $0{ }^{\circ} \mathrm{C}$. The mixture was stirred at $\mathrm{rt}$ for 30 min, diluted with water and extracted with $\mathrm{CH}_{2} \mathrm{Cl}_{2}$. The organic layer was dried over $\mathrm{MgSO}_{4}$, filtered, and concentrated under reduced pressure. The brownish residue was filtered through a short column of silica gel to obtain methyl hydroxy(methyl)carbamodithioate (668 mg, 49\%). Green-white solid. Physical and spectral data were in accordance with literature data. ${ }^{[1] ~}{ }^{1} \mathrm{H}-\mathrm{NMR}\left(300 \mathrm{MHz}, \mathrm{CDCl}_{3}\right) \delta 10.28$ (br s, $\left.1 \mathrm{H}\right), 3.60$ (s, $\left.3 \mathrm{H}\right), 2.64$ (s, $\left.3 \mathrm{H}\right) .{ }^{13} \mathrm{C}-\mathrm{NMR}$ $\left(75 \mathrm{MHz}, \mathrm{CDCl}_{3}\right) \delta 182.42,38.62,19.23$.

$N$-Phenylhydroxylamine. Zinc powder $(28.8 \mathrm{~g}, 440 \mathrm{mmol})$ was added slowly in $\mathrm{HO}^{-\mathrm{NH}}$ portions under vigorous stirring to a solution of ammonium chloride (12.8 g, 240 $\mathrm{mmol}$ ) and nitrobenzene $(24.6 \mathrm{~g}, 200 \mathrm{mmol})$ in $\mathrm{H}_{2} \mathrm{O}(200 \mathrm{~mL})$, while maintaining the temperature below $65^{\circ} \mathrm{C}$. After $3 \mathrm{~h}$ at rt, the reaction mixture was filtered, saturated with 
$\mathrm{HO}^{-\mathrm{N}} \prod_{\mathrm{S}}^{\mathrm{Ph}} \mathrm{S}$

$\mathrm{NaCl}$, extracted with $\mathrm{CH}_{2} \mathrm{Cl}_{2}$ and concentrated to approximately $100 \mathrm{~mL} . \mathrm{N}$ Phenylhydroxylamine (16.8 g, 77\%) was obtained after several precipitations from the organic layer at $-78{ }^{\circ} \mathrm{C}$. White solid (wool-like). Physical and spectral data were in accordance with literature data. ${ }^{[2]}{ }^{1} \mathrm{H}-\mathrm{NMR}\left(300 \mathrm{MHz}, \mathrm{CDCl}_{3}\right) \delta 7.33$ $7.26(\mathrm{~m}, 2 \mathrm{H}), 7.04-6.99(\mathrm{~m}, 3 \mathrm{H}), 6.44$ (br s, 2H). ${ }^{13} \mathrm{C}-\mathrm{NMR}\left(75 \mathrm{MHz}, \mathrm{CDCl}_{3}\right) \delta$ 149.32, 128.93, 122.55, 114.90. IR (neat): 3573, 3312, 3009, 1603, 1495, 1474, $1345 \mathrm{~cm}^{-1}$.

Methyl hydroxy(phenyl)carbamodithioate. Carbon disulfide $(7.67 \mathrm{~g}, 101 \mathrm{mmol})$ and $\mathrm{MeI}$ (14.3 g, $101 \mathrm{mmol})$ were added at $0{ }^{\circ} \mathrm{C}$ to a solution of $\mathrm{N}$-phenyl-hydroxylamine $(10.0 \mathrm{~g}, 91.6$ mmol) in $\mathrm{Et}_{2} \mathrm{O}(400 \mathrm{~mL}) . \mathrm{Et}_{3} \mathrm{~N}(33 \mathrm{~mL}, 458 \mathrm{mmol})$ was added dropwise and the reaction mixture was slowly allowed to warm up to rt and stirred overnight. The reaction mixture was extracted with $5 \% \mathrm{NaHCO}_{3}$, the aqueous layer was acidified to $\mathrm{pH} 3$ with conc. $\mathrm{HCl}$ and extracted with $\mathrm{Et}_{2} \mathrm{O}$. The organic phase was dried over $\mathrm{MgSO}_{4}$ and concentrated under reduced pressure to obtain methyl hydroxy(phenyl)carbamodithioate (10.4 g, 57\%). Yellowwhite solid. ${ }^{1} \mathrm{H}-\mathrm{NMR}\left(300 \mathrm{MHz}, \mathrm{CDCl}_{3}\right) \delta 10.75$ (br s, $\left.1 \mathrm{H}\right), 7.50-7.48$ (m, 5H), 2.61 (s, 3H). ${ }^{13} \mathrm{C}-\mathrm{NMR}\left(75 \mathrm{MHz}, \mathrm{CDCl}_{3}\right) \delta 130.38,129.44,129.07,126.62,124.10,19.55$. IR (neat): 3367, 3064, 2996, 2926, 1594, 1506, 1481, $1368 \mathrm{~cm}^{-1}$. EI-LRMS, m/z (\%): 199 (M, 22), 183 (6), 135 (100), 119 (8), 103 (6), 91 (56), 77 (78), 51 (41). EI-HRMS: calculated for $\mathrm{C}_{8} \mathrm{H}_{9} \mathrm{NOS}_{2}$ : 199.0126; found: 199.0123 .

Methyl adamantylcarbonyloxy(phenyl)carbamodithioate (8). Prepared according to

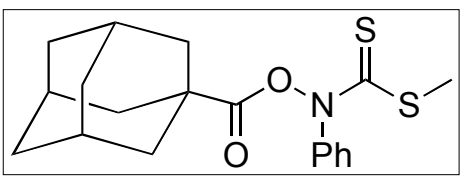
General Procedure A from 1-adamantane carboxylic acid (726 $\mathrm{mg}, 4.03 \mathrm{mmol}$ ), methyl hydroxy(phenyl)carbamodithioate (882 $\mathrm{mg}, 4.43 \mathrm{mmol})$ and $\mathrm{Et}_{3} \mathrm{~N}(0.6 \mathrm{~mL}, 8.30 \mathrm{mmol})$ in $\mathrm{CH}_{2} \mathrm{Cl}_{2}(100$ $\mathrm{mL}$ ). Purification by FC (hexane/EtOAc 99:1) gave 8 (1.06 g, 73\%). Yellow oil. ${ }^{1} \mathrm{H}-\mathrm{NMR}$ (300 MHz, $\left.\mathrm{CDCl}_{3}\right) \delta$ 7.58-7.55 (m, 2H), 7.46-7.39 (m, 3H), $2.60(\mathrm{~s}, 3 \mathrm{H}), 2.05-2.00(\mathrm{~m}, 9 \mathrm{H})$, $1.73(\mathrm{~m}, 6 \mathrm{H}) .{ }^{13} \mathrm{C}-\mathrm{NMR}\left(75 \mathrm{MHz}, \mathrm{CHCl}_{3}\right) \delta$ 197.43, 172.95, 141.38, 129.33, 129.00, 127.60, 38.44, 38.20, 36.04, 27.51, 18.92. IR (neat): 2904, 2850, 1780, 1343, 1211, 1053, 999, 959 $\mathrm{cm}^{-1}$. EI-LRMS, m/z (\%): 361 (M, 1), 317 (21), 302 (20), 270 (37), 150 (87), 135 (68), 93 (71), 77 (100), 67 (43), 51 (50), 41 (45). EI-HRMS: calculated for $\mathrm{C}_{19} \mathrm{H}_{23} \mathrm{NO}_{2} \mathrm{~S}_{2}$ : 361.1170; found: 361.1177. 
Adamantyl methyl phenylcarbonimidodithioate (12). Prepared according to General

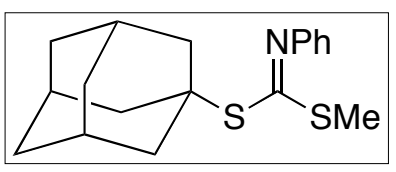
Procedure $\mathrm{C}$ from $8(200 \mathrm{mg}, 0.55 \mathrm{mmol})$ in $\mathrm{CH}_{2} \mathrm{Cl}_{2}(20 \mathrm{~mL})$. Purification by FC (hexane/EtOAc 200:1) gave 12 (143 mg, 82\%). White solid. M.p. $79{ }^{\circ} \mathrm{C} .{ }^{1} \mathrm{H}-\mathrm{NMR}\left(300 \mathrm{MHz}, \mathrm{CDCl}_{3}\right) \delta 7.31(\mathrm{t}$, $J=7.9 \mathrm{~Hz}, 2 \mathrm{H}), 7.07$ (t, $J=7.5 \mathrm{~Hz}, 1 \mathrm{H}), 6.86(\mathrm{~d}, J=7.7 \mathrm{~Hz}, 2 \mathrm{H}), 2.43$ (s, 3H), 2.15 (br s, $6 \mathrm{H}), 2.04(\mathrm{~s}, 3 \mathrm{H}), 1.68(\mathrm{t}, J=13.2 \mathrm{~Hz}, 6 \mathrm{H}) .{ }^{13} \mathrm{C}-\mathrm{NMR}\left(75 \mathrm{MHz}, \mathrm{CHCl}_{3}\right) \delta 158.92,149.75$, $128.65,123.51,120.49,52.86,42.85,36.10,30.08,16.00$. IR (neat): 2907, 2846, 1558, 918 , 889, $695 \mathrm{~cm}^{-1}$. EI-LRMS, m/z (\%): $317\left(\mathrm{M}^{+}, 14\right), 302$ (26), 270 (55), 150 (99), 136 (77), 93 (91), 77 (100), 67 (50), 51 (36). EI-HRMS: calculated for $\mathrm{C}_{18} \mathrm{H}_{23} \mathrm{NS}_{2}$ : 317.1272; found: 317.1272 .

1-Adamantyl azide (4). a) Prepared according to General Procedure D from 8 (500 mg, 1.38

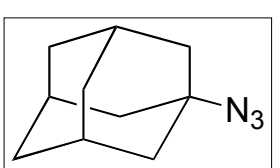
$\mathrm{mmol})$ and benzenesulfonyl azide $(1.52 \mathrm{~g}, 8.30 \mathrm{mmol})$ in $\mathrm{CH}_{2} \mathrm{Cl}_{2}(50 \mathrm{~mL})$. Purification by FC (hexane/EtOAc 99:1) gave 4 (139 mg, 67\%) along with $12(66 \mathrm{mg}, 15 \%)$.

b) Lauryl peroxide was added portionwise every h (16 $\mathrm{mg}, 0.1 \mathrm{eq}, 0.04 \mathrm{mmol})$ to a refluxing solution of adamantyl iodide (100 mg, $0.38 \mathrm{mmol})$ and ethanesulfonyl azide (154 mg, 1.14 mmol) in $6 \mathrm{~mL}$ of a 1:1 mixture of heptane and chlorobenzene under $\mathrm{N}_{2}$. After $5 \mathrm{~h}$, the solvents were removed under reduced pressure and the residue was purified by FC (hexane $100 \%)$ to obtain $47 \mathrm{mg}(0.27 \mathrm{mmol}, 70 \%)$ of 4 . This sample of adamantyl azide was used as reference material for GC analysis. White solid. Physical and spectral data were in accordance with literature data. ${ }^{[3] 1} \mathrm{H}-\mathrm{NMR}\left(300 \mathrm{MHz}, \mathrm{CDCl}_{3}\right) \delta 2.14(\mathrm{~s}, 3 \mathrm{H}), 1.80(\mathrm{~m}, 6 \mathrm{H}), 7.63(\mathrm{~m}, 6 \mathrm{H})$. ${ }^{13} \mathrm{C}-\mathrm{NMR}\left(75 \mathrm{MHz}, \mathrm{CHCl}_{3}\right.$ ) $\delta$ 58.99, 41.50, 35.89, 29.80. IR (neat): 2906, 2851, 2079, 1452 , $1251,1057 \mathrm{~cm}^{-1}$.

1-Tosylpiperidine-4-carboxylic acid. Tosyl chloride $(9.55 \mathrm{~g}, 50.0 \mathrm{mmol})$

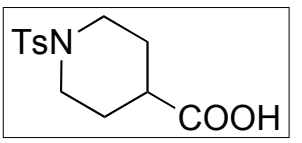

was added to a mixture of piperidine-4-carboxylic acid $(6.45 \mathrm{~g}, 50.0$ mmol) and $\mathrm{NaOH}(4.00 \mathrm{~g}, 100 \mathrm{mmol})$ in $100 \mathrm{~mL}$ of a $1: 1$ mixture of $\mathrm{Et}_{2} \mathrm{O}$ and $\mathrm{H}_{2} \mathrm{O}$. The clear mixture was vigourously stirred at $\mathrm{rt}$ for $6 \mathrm{~h}$ until a white cloudy precipitate was formed. The mixture was diluted with more $\mathrm{Et}_{2} \mathrm{O}$ and $\mathrm{H}_{2} \mathrm{O}$ until the precipitate was completely dissolved and the two layers were separated. The $\mathrm{pH}$ of the aqueous layer was adjusted to 3 by addition of $4 \mathrm{M} \mathrm{HCl}$. The precipitate was filtered off and dissolved in EtOAc. 
The solution was dried over $\mathrm{MgSO}_{4}$ and concentrated under reduced pressure to furnish 1tosylpiperidine-4-carboxylic acid (9.70 g, 68\%). White solid. Physical and spectral data were in accordance with literature data. ${ }^{[4]}{ }^{1} \mathrm{H}-\mathrm{NMR}\left(300 \mathrm{MHz}, \mathrm{CDCl}_{3}\right) \delta 7.63(\mathrm{~d}, J=8.1 \mathrm{~Hz}, 2 \mathrm{H})$, 7.33 (d, $J=8.1 \mathrm{~Hz}, 2 \mathrm{H}), 3.65$ (m, 2H), 2.40-2.48 (m, 5H), 2.23-2.33 (m, 1H), 1.96-1-75 (m, 4H). ${ }^{13} \mathrm{C}-\mathrm{NMR}\left(75 \mathrm{MHz}, \mathrm{CDCl}_{3}\right) \delta 179.99,143.63,132.94,129.65,127.59,45.28,39.74$, 27.11, 21.47. IR (neat): $3019,1709,1216,1162,758 \mathrm{~cm}^{-1}$.

Methyl phenyl(1-tosylpiperidine-4-carbonyloxy)carbamodithioate (9). Prepared according

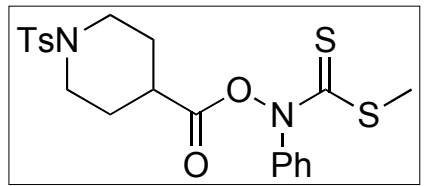
to General Procedure A from 1-tosylpiperidine-4-carboxylic acid (187 mg, $0.66 \mathrm{mmol}$ ), methyl hydroxy(phenyl)carbamodithioate (145 mg, $0.73 \mathrm{mmol})$ and $\mathrm{Et}_{3} \mathrm{~N}(0.1 \mathrm{~mL}, 1.32 \mathrm{mmol})$ in $\mathrm{CH}_{2} \mathrm{Cl}_{2}(20$ $\mathrm{mL}$ ). Purification by FC (hexane/EtOAc 3:1) gave 9 (258 mg, 81\%). Yellow oil. ${ }^{1} \mathrm{H}-\mathrm{NMR}$ $\left(300 \mathrm{MHz}, \mathrm{CDCl}_{3}\right) \delta 7.63(\mathrm{~d}, J=2.5 \mathrm{~Hz}, 2 \mathrm{H}), 7.52-7.43(\mathrm{~m}, 5 \mathrm{H}), 7.32(\mathrm{~d}, J=2.5 \mathrm{~Hz}, 2 \mathrm{H})$, 3.63-3.56 (m, 2H), 2.61-2.47 (m, 6H), $2.44(\mathrm{~s}, 3 \mathrm{H}), 2.10-1.88(\mathrm{~m}, 4 \mathrm{H}) .{ }^{13} \mathrm{C}-\mathrm{NMR}(75 \mathrm{MHz}$, $\left.\mathrm{CDCl}_{3}\right) \delta 197.42,170.17,143.67,140.90,132.99,130.17,129.68,129.43,128.15,127.60$, 127.57, 44.91, 38.01, 27.04, 21.49, 19.35. IR (neat): 2949, 2926, 2856, 1781, 1370, 1332, 1159, 928, 728, $689 \mathrm{~cm}^{-1}$. ESI-LRMS, $m / z(\%): 487\left(\mathrm{M}+\mathrm{Na}^{+}, 8\right), 476(69), 421(9), 381(23)$, 313 (8), 182 (38), 132 (100). ESI-HRMS: calculated for $\mathrm{C}_{21} \mathrm{H}_{24} \mathrm{~N}_{2} \mathrm{O}_{4} \mathrm{~S}_{3} \mathrm{Na}(\mathrm{M}+\mathrm{Na})^{+}$: 487.0795; found: 487.0790 .

Methyl 1-tosylpiperidin-4-yl phenylcarbonimidodithioate (13). Prepared according to

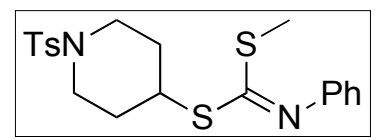
General Procedure C from $9(100 \mathrm{mg}, 0.20 \mathrm{mmol})$ in $\mathrm{CH}_{2} \mathrm{Cl}_{2}(10 \mathrm{~mL})$. Purification by FC (hexane/EtOAc 8:1) gave 13 (71 $\mathrm{mg}, 84 \%$ ). Colorless oil. ${ }^{1} \mathrm{H}-\mathrm{NMR}\left(300 \mathrm{MHz}, \mathrm{CDCl}_{3}\right) \delta$ 7.64-7.61 (m, 2H), 7.33$7.29(\mathrm{~m}, 5 \mathrm{H}), 7.09-7.04(\mathrm{~m}, 1 \mathrm{H}), 6.81-6.78(\mathrm{~m}, 2 \mathrm{H}), 3.62-3.58(\mathrm{~m}, 3 \mathrm{H}), 2.57-2.42(\mathrm{~m}, 8 \mathrm{H})$, 2.17-2.13 (m, 2H), 1.78-1.76 (m, 2H). ${ }^{13} \mathrm{C}-\mathrm{NMR}\left(75 \mathrm{MHz}, \mathrm{CHCl}_{3}\right) \delta$ 149.51, 143.53, 132.88, $129.59,128.86,127.54,123.89,120.03,45.72,41.28,31.31,21.40,14.85$. IR (neat): 2925, 2844, 1564, 1333, 1159, 925, $722 \mathrm{~cm}^{-1}$. ESI-LRMS, $m / z(\%): 421\left(\mathrm{M}+\mathrm{H}^{+}, 40\right), 381(100)$, 353 (19), 313 (7), 240 (27), 155 (13). ESI-HRMS: calculated for $\mathrm{C}_{20} \mathrm{H}_{24} \mathrm{~N}_{2} \mathrm{O}_{2} \mathrm{~S}_{2} \mathrm{Na}(\mathrm{M}+\mathrm{Na})^{+}$: 421.1078; found: 421.1085 . 
4-Azido-1-tosylpiperidine (16). Prepared according to General Procedure D from 9 (500 mg, $\stackrel{\mathrm{TsN}}{ }$ $1.08 \mathrm{mmol})$ and benzenesulfonyl azide $(1.18 \mathrm{~g}, 6.46 \mathrm{mmol})$ in $\mathrm{CH}_{2} \mathrm{Cl}_{2}$ (50 $\mathrm{mL}$ ). Purification by FC (hexane/EtOAc 100:1) gave a mixture of $\mathbf{1 6}$ (115 mg, $38 \%$ ) contaminated by $13(132 \mathrm{mg}, 29 \%)$. A pure sample was obtained after a second FC (hexane/EtOAc 200:1). White solid. Physical and spectral data were in accordance with literature data. ${ }^{[5]}{ }^{1} \mathrm{H}-\mathrm{NMR}\left(300 \mathrm{MHz}, \mathrm{CDCl}_{3}\right) \delta 7.64(\mathrm{~d}, J=8.3 \mathrm{~Hz}, 2 \mathrm{H}), 7.33(\mathrm{~m}, J=$ $7.9 \mathrm{~Hz}, 2 \mathrm{H}), 3.54-3.47$ (m, 1H), 3.29-3.22 (m, 2H), 2.93-2.85 (m, 2H), 2.44 (s, 3H), 1.99-1.90 (m, 2H), 1.78-1.67 (m, 2H). ${ }^{13} \mathrm{C}-\mathrm{NMR}\left(75 \mathrm{MHz}, \mathrm{CHCl}_{3}\right) \delta 143.71,132.93,129.71,127.57$, 56.01, 43.30, 29.83, 21.50. IR (neat): 2929, 2857, 2099, 1334, 1246, $1159 \mathrm{~cm}^{-1}$.

2-Methyl-3-(naphthalen-2-yl)propanoic acid. $n$-Butyllithium $(16.3 \mathrm{~mL}$ of a $2.45 \mathrm{M}$ solution

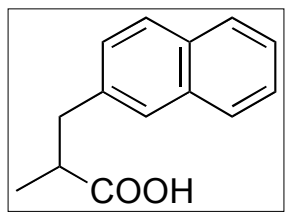
in hexane, $40.0 \mathrm{mmol}$ ) was added at $0{ }^{\circ} \mathrm{C}$ to a solution of diisopropylamine $(5.65 \mathrm{~mL}, 40.0 \mathrm{mmol})$ in THF $(20 \mathrm{~mL})$ and the reaction mixture was stirred for $15 \mathrm{~min}$. Propionic acid (1.49 mL, $20.0 \mathrm{mmol})$ in THF $(5 \mathrm{~mL})$ was added and the reaction mixture was stirred for $30 \mathrm{~min}$ at $0{ }^{\circ} \mathrm{C}$. Benzyl bromide (4.42 g, $20.0 \mathrm{mmol}$ ) in THF ( $5 \mathrm{~mL}$ ) was slowly added over a period of $5 \mathrm{~min}$ and the reaction mixture was stirred at $0{ }^{\circ} \mathrm{C}$ for $0.5 \mathrm{~h}$ and at $\mathrm{rt}$ for $2 \mathrm{~h}$. It was then acidified with $4 \mathrm{M}$ $\mathrm{HCl}$ to $\mathrm{pH} \mathrm{2,} \mathrm{saturated} \mathrm{with} \mathrm{NaCl}$, and extracted with EtOAc. The combined organic layers were extracted with $5 \% \mathrm{NaHCO}_{3}$, the aqueous extracts were combined, acidified to $\mathrm{pH} 2$ with $4 \mathrm{M} \mathrm{HCl}$ and extracted with EtOAc to give 2-methyl-3-(naphthalen-2-yl)propanoic acid (2.40 g, 56\%). White solid. Physical and spectral data were in accordance with literature data. ${ }^{[6]}{ }^{1} \mathrm{H}-$ NMR (300 MHz, $\left.\mathrm{CDCl}_{3}\right) \delta 10.77$ (br s, 1H), 7.83-7.77 (m, 3H), 7.65 (s, 1H), 7.49-7.42 (m, 2H), $7.33(\mathrm{dd}, J=8.4,1.8 \mathrm{~Hz}, 1 \mathrm{H}), 3.29-3.23(\mathrm{~m}, 1 \mathrm{H}), 2.92-2.80(\mathrm{~m}, 2 \mathrm{H}), 1.23(\mathrm{~d}, J=6.8$ $\mathrm{Hz}, 3 \mathrm{H}) .{ }^{13} \mathrm{C}-\mathrm{NMR}\left(75 \mathrm{MHz}, \mathrm{CDCl}_{3}\right) \delta 182.74,136.52,133.43,132.16,128.01,127.56$, 127.50, 127.43, 127.30, 125.95, 125.39, 41.17, 39.36, 16.46. IR (neat): 2939, 2361, 1695, $1465,1418,1287,1234,814,743 \mathrm{~cm}^{-1}$.

Methyl 2-methyl-3-(naphthalen-2-yl)propanoyloxy(phenyl)carbamodithioate (10).<smiles>CSC(=S)N(OC(=O)C(C)Cc1ccc2ccccc2c1)Pc1ccccc1</smiles>
Prepared according to General Procedure B from methyl hydroxy(phenyl)carbamodithioate $(185 \mathrm{mg}, 0.30 \mathrm{mmol}), \mathrm{DCC}$ (213 mg, $1.03 \mathrm{mmol}$ ), DMAP (11 mg, $0.09 \mathrm{mmol}$ ) and 2methyl-3-(naphthalen-2-yl)propanoic acid (200 mg, 0.93 
mmol) in $\mathrm{CH}_{2} \mathrm{Cl}_{2}(20 \mathrm{~mL})$. Purification by $\mathrm{FC}$ (hexane/EtOAc 20:1) gave 10 (287 mg, 77\%). Yellow oil. ${ }^{1} \mathrm{H}-\mathrm{NMR}\left(300 \mathrm{MHz}, \mathrm{CDCl}_{3}\right) \delta$ 7.70-7.66 (m, 1H), 7.60-7.56 (m, 2H), 7.42-7.30 (m, 5H), 7.23-7.18 (m, 3H), $7.13(\mathrm{dd}, J=1.8,8.3 \mathrm{~Hz}, 1 \mathrm{H}), 3.15(\mathrm{dd}, J=7.6,13.3 \mathrm{~Hz}, 1 \mathrm{H})$, 3.04-2.92 (m, 1H), $2.84(\mathrm{dd}, J=7.0,13.3 \mathrm{~Hz}, 1 \mathrm{H}), 2.40(\mathrm{~s}, 3 \mathrm{H}), 1.24(\mathrm{~d}, J=6.8 \mathrm{~Hz}, 3 \mathrm{H}) .{ }^{13} \mathrm{C}-$ NMR $\left(75 \mathrm{MHz}, \mathrm{CDCl}_{3}\right) \delta 197.69,172.34,141.07,135.44,133.31,132.18,129.63,129.08$, $128.05,127.81,127.60,127.52,127.49,127.05,125.92,125.48,39.54,39.33,19.05,16.72$. IR (neat): 3053, 2973, 2916, 1788, 1353, 1065, 1026, $957 \mathrm{~cm}^{-1}$. ESI-LRMS, $\mathrm{m} / z(\%): 418$ $\left(\mathrm{M}+\mathrm{Na}^{+}, 65\right), 396\left(\mathrm{M}+\mathrm{H}^{+}, 33\right), 381$ (14), 182 (100), 167 (34), 135 (25). ESI-HRMS: calculated for $\mathrm{C}_{22} \mathrm{H}_{22} \mathrm{NO}_{2} \mathrm{~S}_{2}(\mathrm{M}+\mathrm{H})^{+}$: 396.1091; found: 396.1097 .

Methyl 1-(naphthalen-2-yl)propan-2-yl phenylcarbonimidodithioate (14). Prepared

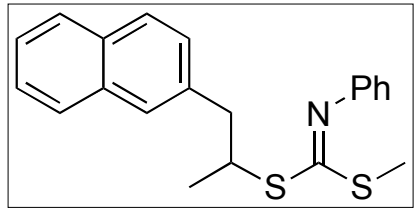
according to General Procedure C from 10 (100 $\mathrm{mg}, 0.25 \mathrm{mmol})$ in $\mathrm{CH}_{2} \mathrm{Cl}_{2}(10 \mathrm{~mL})$. Purification by FC (hexane/EtOAc 66:1) gave 14 (70 mg, 76\%). Colorless oil. ${ }^{1} \mathrm{H}-\mathrm{NMR}\left(300 \mathrm{MHz}, \mathrm{CDCl}_{3}\right) \delta 7.83-$ 7.77 (m, 3H), $7.64(\mathrm{~s}, 1 \mathrm{H})$, 7.49-7.42 (m, 2H), 7.37-7.31 (m, 3H), 7.13-7.09 (m, 1H), 6.89$6.87(\mathrm{~m}, 2 \mathrm{H}), 4.08$ (br s, 1H), 3.36 (br s, $1 \mathrm{H}), 2.85$ (br s, $1 \mathrm{H}), 2.49(\mathrm{~s}, 3 \mathrm{H}), 1.31(\mathrm{~s}, 3 \mathrm{H}) .{ }^{13} \mathrm{C}-$ NMR (75 MHz, $\left.\mathrm{CHCl}_{3}\right) \delta 161.11,149.90,136.39,133.37,132.26,128.91,127.89,127.73$, 127.58, 127.53, 125.93, 125.41, 123.82, 120.32, 43.37, 42.70, 19.82, 15.04. IR (neat): 3053, 2968, 2923, 1642, 1564, 1206, 924, 754, $694 \mathrm{~cm}^{-1}$ ESI-LRMS, $m / z(\%): 352\left(\mathrm{M}+\mathrm{H}^{+}, 2\right), 313$ (1), 212 (15), 182 (10), 169 (14), 164 (2), 150 (20), 141 (6), 133 (100). ESI-HRMS: calculated for $\mathrm{C}_{21} \mathrm{H}_{22} \mathrm{NS}_{2}$ : 352.1193 ; found: 352.1211 .

2-(2-Azidopropyl)naphthalene (17). Prepared according to General

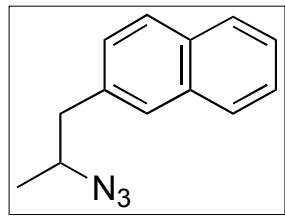
Procedure D from 10 (200 mg, $0.50 \mathrm{mmol})$ and benzenesulfonyl azide (550 $\mathrm{mg}, 3.00 \mathrm{mmol})$ in $\mathrm{CH}_{2} \mathrm{Cl}_{2}(20 \mathrm{~mL})$. Purification by $\mathrm{FC}$ (hexane/EtOAc 50:1) gave a mixture of $17(56 \mathrm{mg}, 53 \%)$ contaminated by 14 (56 mg, $32 \%$ ). An analytical sample was obtained after a second FC (hexane/EtOAc 100:1). Colorless oil. ${ }^{1} \mathrm{H}-\mathrm{NMR}\left(300 \mathrm{MHz}, \mathrm{CDCl}_{3}\right) \delta$ 7.85-7.80 (m, 3H), 7.67 (s, 1H), 7.51-7.43 (m, 2H), 7.34 $(\mathrm{dd}, J=1.7,8.5 \mathrm{~Hz}, 1 \mathrm{H}), 3.86-3.75(\mathrm{~m}, 1 \mathrm{~Hz}), 2.89(\mathrm{dd}, J=7.1,13.7 \mathrm{~Hz}, 1 \mathrm{H}), 3.02(\mathrm{dd}, J=$ 6.5, $13.7 \mathrm{~Hz}, 1 \mathrm{H}), 1.31(\mathrm{~d}, J=6.6 \mathrm{~Hz}, 3 \mathrm{H}) .{ }^{13} \mathrm{C}-\mathrm{NMR}\left(75 \mathrm{MHz}, \mathrm{CHCl}_{3}\right) \delta 135.25,133.50$, $132.35,128.11,127.87,127.64,127.55,127.47,126.10,125.59,58.94,42.68,19.14$. IR (neat): 3050, 2965, 2923, 2100, 1641, 1564, 1207, 924, 864, 816, 753, $694 \mathrm{~cm}^{-1}$. EI-LRMS 
m/z (\%): $211\left(\mathrm{M}^{+}, 43\right), 183$ (12), 164 (66), 150 (100), 136 (100), 119 (27), 77 (91), 51 (41). EI-HRMS calculated for $\mathrm{C}_{13} \mathrm{H}_{13} \mathrm{~N}_{3}$ : 211.1109; found: 211.1101 .

Ethyl 3-(dimethylphenylsilyl)-2-methylpropanoate. Granulated lithium pieces (210 mg, $\mathrm{M}_{\mathrm{O}}^{\mathrm{PhMe}_{2} \mathrm{Si}} \mathrm{OEt}$ $30.0 \mathrm{mmol}$ ) were stirred for $15 \mathrm{~min}$ in hexane. The solvent was removed and replaced by THF $(20 \mathrm{~mL})$. Chlorodimethylphenylsilane $(1.7 \mathrm{~g}, 10.0$ mmol) was added at $0{ }^{\circ} \mathrm{C}$ and the reaction mixture was stirred at that temperature overnight to give a deep red solution. Copper cyanide (448 mg, $5.00 \mathrm{mmol}$ ) was added at $-20{ }^{\circ} \mathrm{C}$ and the resulting mixture was stirred at $-20{ }^{\circ} \mathrm{C}$ for 60 min and was then cooled to $-78{ }^{\circ} \mathrm{C}$. Chlorotrimethylsilane $(1.36 \mathrm{~g}, 12.5 \mathrm{mmol})$ and ethyl methacrylate $(476 \mathrm{mg}$, $4.17 \mathrm{mmol})$ in THF $(6 \mathrm{~mL})$ were added, the reaction mixture was stirred at $-78^{\circ} \mathrm{C}$ for $30 \mathrm{~min}$, treated with saturated $\mathrm{NH}_{4} \mathrm{Cl}$, and extracted with $t$-BuOMe. The organic layer was washed with saturated $\mathrm{NH}_{4} \mathrm{Cl}$ and brine, dried with $\mathrm{MgSO}_{4}$, and concentrated. The residue was dissolved in THF (44 mL) and a solution of $\mathrm{Bu}_{4} \mathrm{NF}$ in THF (18 $\mathrm{mL}$ of a $1 \mathrm{M}$ solution) was slowly added. After stirring at $\mathrm{rt}$ for $2 \mathrm{~h}, \mathrm{H}_{2} \mathrm{O}$ was added and the mixture was extracted with $t$ $\mathrm{BuOMe}$, dried with $\mathrm{MgSO}_{4}$ and concentrated. Purification by $\mathrm{FC}$ (cyclohexane/t-BuOMe 50:1) gave ethyl 3-(dimethylphenylsilyl)-2-methylpropanoate (864 mg, 83\%). Colorless oil. ${ }^{1} \mathrm{H}-\mathrm{NMR}\left(300 \mathrm{MHz}, \mathrm{CDCl}_{3}\right)$ d 7.5-7.48 (m, 2H), 7.37-7.33 (m, 3H), 4.06-3.92 (m, 2H), 2.58$2.46(\mathrm{~m}, 1 \mathrm{H}), 1.29$ (dd, $J=7.5,14.8 \mathrm{~Hz}, 1 \mathrm{H}), 1.20(\mathrm{t}, J=7.1 \mathrm{~Hz}, 3 \mathrm{H}), 1.14(\mathrm{~d}, J=6.8 \mathrm{~Hz}$, $3 \mathrm{H}), 0.91(\mathrm{dd}, J=7.1,14.8 \mathrm{~Hz}, 1 \mathrm{H}), 0.30(\mathrm{~s}, 6 \mathrm{H}) .{ }^{13} \mathrm{C}-\mathrm{NMR}\left(75 \mathrm{MHz}, \mathrm{CHCl}_{3}\right) \delta 177.39$, $138.73,133.48,128.90,127.70,60.10,35.58,20.69,20.63,14.06,-2.58$. IR (neat): 2970, 2935, 2906, 1730, 1249, 1197, 1147, 1112, 831, 725, $698 \mathrm{~cm}^{-1}$. EI-LRMS, $m / z(\%): 250\left(\mathrm{M}^{+}\right.$, 0.6), 235 (72), 207 (26), 173 (52), 145 (30), 135 (100), 105 (18), 75 (24). ESI-HRMS: calculated for $\mathrm{C}_{14} \mathrm{H}_{22} \mathrm{O}_{2} \mathrm{SiNa}(\mathrm{M}+\mathrm{Na})^{+}:$273.1286; found, 273.1280 .

3-(Dimethylphenylsilyl)-2-methylpropanoic acid. $\mathrm{KOH}(330 \mathrm{mg}, 5.88 \mathrm{mmol})$ was added

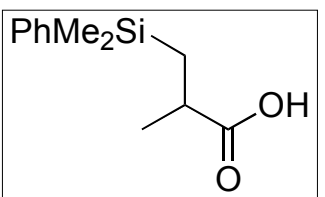
to a solution of ethyl 3-(dimethylphenylsilyl)-2-methylpropanoate (490 $\mathrm{mg}, 1.96 \mathrm{mmol})$ in a mixture of $\mathrm{MeOH}(5 \mathrm{~mL})$ and $\mathrm{H}_{2} \mathrm{O}(0.5 \mathrm{~mL})$ and the resulting mixture was heated under reflux for $5 \mathrm{~h}$. The reaction mixture was diluted with a $0.5 \mathrm{M}$ solution of $\mathrm{KOH}$ and washed with $t$-BuOMe. The aqueous layer was acidified with $4 \mathrm{M} \mathrm{HCl}$ and extracted with $t$-BuOMe. The organic layer was dried with $\mathrm{MgSO}_{4}$ and concentrated under reduced pressure to give the desired acid (428 $\mathrm{mg}, 98 \%$ ). 
Colorless oil. ${ }^{1} \mathrm{H}-\mathrm{NMR}\left(300 \mathrm{MHz}, \mathrm{CDCl}_{3}\right) \delta 11.80$ (br s, $\left.1 \mathrm{H}\right), 7.60-7.54$ (m, 2H), 7.42-7.37 (m, 3H), 2.65-2.54 (m, 1H), $1.36(\mathrm{dd}, J=7.0,14.7 \mathrm{~Hz}, 1 \mathrm{H}), 1.21(\mathrm{~d}, J=7.0 \mathrm{~Hz}, 3 \mathrm{H}), 0.99$ $(\mathrm{dd}, J=7.7,14.7 \mathrm{~Hz}, 1 \mathrm{H}), 0.37(\mathrm{~s}, 6 \mathrm{H}) .{ }^{13} \mathrm{C}-\mathrm{NMR}\left(75 \mathrm{MHz}, \mathrm{CHCl}_{3}\right) \delta 184.43,138.52$, 133.51, 129.02, 127.80, 35.51, 20.57, 20.11, -2.47, -2.63. IR (neat): 2970, 1701, 1248, 1112 , 820, $698 \mathrm{~cm}^{-1}$. ESI-LRMS, $m / z(\%): 245\left(\mathrm{M}+\mathrm{Na}^{+}, 76\right), 167$ (5), 145 (100), 132 (22). ESIHRMS: calculated for $\mathrm{C}_{12} \mathrm{H}_{18} \mathrm{O}_{2} \mathrm{SiNa}(\mathrm{M}+\mathrm{Na})^{+}: 245.0973$; found: 245.0974 .

Methyl 3-(dimethyl(phenyl)silyl)-2-methylpropanoyloxy(phenyl)carbamodithioate (11).<smiles>CSC(=S)N(OC(=O)C(C)C[SiH2]C(C)C)c1ccccc1</smiles>
Prepared according to General Procedure B from 3(dimethylphenylsilyl)-2-methylpropanoic acid (1.31 g, 5.90 mmol), methyl hydroxy(phenyl)carbamodithioate (1.12 g, 5.90 mmol), DCC (1.45 g, $7.10 \mathrm{mmol})$ and DMAP (73 mg, $0.60 \mathrm{mmol})$ in $\mathrm{CH}_{2} \mathrm{Cl}_{2}(100 \mathrm{~mL})$. Purification by FC (cyclohexane/t-BuOMe 50:1) gave 11 (1.52 g, 64\%). Yellow oil. ${ }^{1} \mathrm{H}-\mathrm{NMR}$ $\left(300 \mathrm{MHz}, \mathrm{CDCl}_{3}\right) \delta$ 7.56-7.53 (m, 2H), 7.49-7.42 (m, 5H), 7.36-7.33 (m, 3H), 2.73-2.62 (m, $1 \mathrm{H}), 2.60$ (s, 3H), $1.36(\mathrm{dd}, J=6.1,14.9 \mathrm{~Hz}, 1 \mathrm{H}), 1.23$ (d, $J=6.8 \mathrm{~Hz}, 3 \mathrm{H}), 0.97$ (dd, $J=8.3$, $14.8 \mathrm{~Hz}, 1 \mathrm{H}), 0.22$ (s, 6H). ${ }^{13} \mathrm{C}-\mathrm{NMR}\left(75 \mathrm{MHz}, \mathrm{CHCl}_{3}\right) \delta 197.23,173.15,141.00,137.76$, $133.17,129.59,129.03,128.90,127.98,127.64,33.33,19.87,19.74,18.92,-2.56,-2.99$. IR (neat): 2956, 2921, 1791, 1353, 1066, 829, $692 \mathrm{~cm}^{-1}$. EI-LRMS, $m / z(\%): 403\left(\mathrm{M}^{+}, 0.5\right), 359$ (1), 302 (2), 270 (22), 205 (42), 181 (71), 148 (70), 135 (100), 108 (65), 77 (56). EI-HRMS: calculated for $\mathrm{C}_{20} \mathrm{H}_{25} \mathrm{NO}_{2} \mathrm{~S}_{2} \mathrm{Si}$ : 403.1096, found: 403.1099 .

1-(Dimethyl(phenyl)silyl)propan-2-yl methyl phenylcarbonimidodithioate (15). Prepared

$\underset{\mathrm{S}_{19} \mathrm{PhMe}_{2} \mathrm{Si}}{\mathrm{C}_{25} \mathrm{H}_{25} \mathrm{NS}_{2} \mathrm{Si}^{-}} \mathrm{N}_{35.63}^{\mathrm{N}^{-\mathrm{Ph}}}$
according to General Procedure $\mathrm{C}$ from $11(85 \mathrm{mg}, 0.21 \mathrm{mmol})$ in $\mathrm{CH}_{2} \mathrm{Cl}_{2}(8 \mathrm{~mL})$. Purification by $\mathrm{FC}$ (hexane/EtOAc 50:1) gave 15 (50 mg, 66\%). Colorless oil. ${ }^{1} \mathrm{H}-\mathrm{NMR}\left(300 \mathrm{MHz}^{\mathrm{CDCl}}{ }_{3}\right) \delta$ 7.52-7.49 (m, $2 \mathrm{H}), 7.37-7.26(\mathrm{~m}, 5 \mathrm{H}), 7.10-7.05(\mathrm{~m}, 1 \mathrm{H}), 6.86-6.83(\mathrm{~m}, 2 \mathrm{H}), 3.81(\mathrm{br}$ $\mathrm{s}, 1 \mathrm{H}), 2.44(\mathrm{~s}, 3 \mathrm{H}), 1.47-1.32(\mathrm{~m}, 4 \mathrm{H}), 1.19-1.07(\mathrm{~m}, 1 \mathrm{H}), 0.36(\mathrm{~s}, 3 \mathrm{H}), 0.34(\mathrm{~s}, 3 \mathrm{H}) .{ }^{13} \mathrm{C}-$ NMR $\left(75 \mathrm{MHz}, \mathrm{CHCl}_{3}\right) \delta 133.53,129.04,128.95,128.88,127.81,127.78,123.72,120.29$, 39.66, 24.75, 15.00, 13.55, -2.12, -2.27. IR (neat): 3064, 2956, 2921, 1564, 1484, 1427, 1248, 1207, 1112, 924, 827, $692 \mathrm{~cm}^{-1}$. EI-LRMS, m/z (\%): $359\left(\mathrm{M}^{+}, 1\right), 302$ (1), 270 (1), 224 (9), 183 (30), 177 (19), 167 (31), 150 (25), 135 (100), 105 (25). EI-HRMS: calculated for $\mathrm{C}_{19} \mathrm{H}_{25} \mathrm{NS}_{2} \mathrm{Si}: 359.1198$, found: 359.1199 . 
(2-Azidopropyl)dimethyl(phenyl)silane (18). Prepared according to General Procedure D

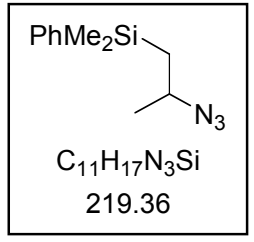

from 11 (200 mg, $0.50 \mathrm{mmol}$ ) and benzenesulfonyl azide (545 $\mathrm{mg}$, $2.97 \mathrm{mmol}$ ) in $\mathrm{CH}_{2} \mathrm{Cl}_{2}(20 \mathrm{~mL})$. Purification by $\mathrm{FC}$ (cyclohexane $/ t-\mathrm{BuOMe}$ 50:1) gave a mixture of 18 (57 mg, 52\%) contaminated by 15 (47 mg, 26\%). ${ }^{1} \mathrm{H}-\mathrm{NMR}\left(300 \mathrm{MHz}, \mathrm{CDCl}_{3}\right) \delta$ 7.56-7.50 (m, 2H), 7.41-7.36 (m, 3H), 3.57$3.45(\mathrm{~m}, 1 \mathrm{H}), 1.08(\mathrm{~d}, J=6.4 \mathrm{~Hz}, 2 \mathrm{H}), 1.07(\mathrm{dd}, J=7.3,14.6 \mathrm{~Hz}, 1 \mathrm{H}), 0.91(\mathrm{dd}, J=7.5,14.6$ $\mathrm{Hz}, 1 \mathrm{H}), 0.38$ (s, 3H), 0.37 (s, 3H). ${ }^{13} \mathrm{C}-\mathrm{NMR}\left(75 \mathrm{MHz}, \mathrm{CHCl}_{3}\right) \delta 138.18,133.45,129.11$, 127.86, 72.69, 49.35, 26.91. IR (neat): 3065, 2957, 2923, 2096, 1250, 1113, $830 \mathrm{~cm}^{-1}$. HRMS: Determination of the exact mass was not possible due to the instability of the azide group.

tert-Butyl 2-((methyl(methylthiocarbonothioyl)aminooxy)carbonyl)pyrrolidine-1-

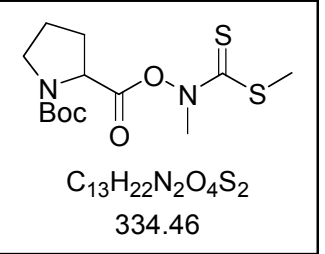
carboxylate (24). Prepared according to General Procedure B from $\mathrm{N}$ Boc-proline (160 mg, $0.74 \mathrm{mmol}$ ), DCC (169 mg, $0.82 \mathrm{mmol}$ ), DMAP ( 9 $\mathrm{mg}, 0.07 \mathrm{mmol}$ ) and methyl hydroxy(methyl)carbamodithioate (100 mg, $0.74 \mathrm{mmol})$ in $\mathrm{CH}_{2} \mathrm{Cl}_{2}(10 \mathrm{~mL})$. Purification by $\mathrm{FC}$ (hexane/EtOAc 5:1) gave 24 (210 mg, 86\%). Yellow oil. ${ }^{1} \mathrm{H}-\mathrm{NMR}\left(300 \mathrm{MHz}, \mathrm{CDCl}_{3}\right) \delta$ 4.43-4.38 (m, 1H), 3.75 $(\mathrm{s}, 3 \mathrm{H}$ from minor rotamer), $3.73(\mathrm{~s}, 3 \mathrm{H}$ from major rotamer), 3.58-3.33 $(\mathrm{m}, 2 \mathrm{H}), 2.51(\mathrm{~s}, 3 \mathrm{H}$ from minor rotamer), 2.50 ( $\mathrm{s}, 3 \mathrm{H}$ from major rotamer), 2.35-2.22 (m, 2H), 2.14-1.88 $(\mathrm{m}, 2 \mathrm{H})$, $1.42\left(\mathrm{~s}, 9 \mathrm{H}\right.$ from major rotamer), 1.41 (s, 9H from minor rotamer). ${ }^{13} \mathrm{C}-\mathrm{NMR}$ (major rotamer, $\left.75 \mathrm{MHz}, \mathrm{CHCl}_{3}\right) \delta 196.36,169.12,154.15,80.46,57.36,46.36,42.46,29.28,28.18,24.52$, 18.44. ${ }^{13} \mathrm{C}-\mathrm{NMR}$ (minor rotamer, $75 \mathrm{MHz}, \mathrm{CHCl}_{3}$ ) $\delta$ 196.73, 169.06, 153.14, 80.25, 57.23, 46.15, 42.44, 30.43, 28.24, 23.41, 18.50. IR (neat): 2975, 2930, 1799, 1688, 1390, 1364, 1161, $1060 \mathrm{~cm}^{-1}$. ESI-LRMS, m/z (\%): $357\left(\mathrm{M}+\mathrm{Na}^{+}, 72\right), 162$ (36), 138 (100), 133 (25). ESIHRMS: calculated for $\mathrm{C}_{13} \mathrm{H}_{22} \mathrm{~N}_{2} \mathrm{O}_{4} \mathrm{~S}_{2} \mathrm{Na}(\mathrm{M}+\mathrm{Na})^{+}$: 357.0918 ; found: 357.0926 .

tert-Butyl 2-azidopyrrolidine-1-carboxylate (27). Prepared according to General Method E

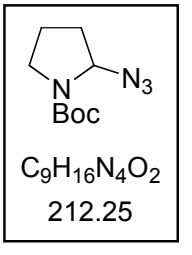
from 24 (200 mg, $0.60 \mathrm{mmol})$, benzenesulfonyl azide (663 mg, $3.62 \mathrm{mmol}$ ) and AIBN (392 mg, $2.40 \mathrm{mmol}$, added portionwise every $\mathrm{h}$ ) in benzene $(10 \mathrm{~mL})$ for 8 h. Purification by FC (cyclohexane/EtOAc 15:1) gave 27 (78 mg, 61\%). Colorless oil. Physical and spectral data were in accordance with literature data. ${ }^{[7]}$ ${ }^{1} \mathrm{H}-\mathrm{NMR}\left(300 \mathrm{MHz}, \mathrm{CDCl}_{3}\right) \delta$ 5.55-5.44 (m, 1H), 3.54-3.45 (m, 1H), 3.33-3-30 (m, 1H), 
1.93-1.88 (m, 4H), $1.50\left(\mathrm{~s}, 9 \mathrm{H}\right.$ from major rotamer), 1.47 (s, 9H from minor rotamer). ${ }^{13} \mathrm{C}$ NMR (major rotamer, $75 \mathrm{MHz}, \mathrm{CHCl}_{3}$ ) $\delta$ 154.62, 81.12, 74.44, 45.82, 33.31, 28.23, 22.01. ${ }^{13} \mathrm{C}$-NMR (minor rotamer, $75 \mathrm{MHz}, \mathrm{CHCl}_{3}$ ) $\delta$ 153.62, 80.42, 74.44, 45.82, 32.38, 28.23, 22.96. IR (neat): 2979, 2935, 2889, 2106, 1698, 1382, 1366, $1159 \mathrm{~cm}^{-1}$. EI-LRMS, $m / z(\%)$ : $212\left(\mathrm{M}^{+}, 1\right), 170(27), 114$ (66), 70 (77), 57 (100), 41 (79).

2-Phenethoxypropanoic acid. 2-Phenylethanol (2.04 g, $16.7 \mathrm{mmol})$ was added to a solution $\mathrm{C}_{\substack{11 \\ 194.23}}^{\mathrm{COOH}}$ of $\mathrm{NaH}(1.24 \mathrm{~g}, 31 \mathrm{mmol}$ of a $60 \%$ dispersion in oil) in THF $(15 \mathrm{~mL})$ at $0{ }^{\circ} \mathrm{C}$. The resulting mixture was stirred for $30 \mathrm{~min}$ and 2bromopropionic acid $(2.29 \mathrm{~g}, 15.0 \mathrm{mmol})$ was added slowly at $0{ }^{\circ} \mathrm{C}$. The reaction mixture was heated under reflux for $4 \mathrm{~h}$. After cooling down to $\mathrm{rt}, \mathrm{H}_{2} \mathrm{O}$ and $t$-BuOMe were added and the resulting mixture was extracted with sat. $\mathrm{NaHCO}_{3}$. The aqueous extract was carefully acidified with sat. citric acid and extracted with $t$-BuOMe. Drying with $\mathrm{MgSO}_{4}$ and concentration under reduced pressure gave 2phenethoxypropanoic acid (2.74 g, 94\%). Colorless oil. ${ }^{1} \mathrm{H}-\mathrm{NMR}\left(300 \mathrm{MHz}, \mathrm{CDCl}_{3}\right) \delta 7.34$ $7.29(\mathrm{~m}, 2 \mathrm{H}), 7.25-7.20(\mathrm{~m}, 3 \mathrm{H}), 3.99(\mathrm{q}, J=6.9 \mathrm{~Hz}, 1 \mathrm{H}), 3.83-3.70(\mathrm{~m}, 1 \mathrm{H}), 2.94(\mathrm{t}, J=7.1$ $\mathrm{Hz}, 2 \mathrm{H}), 1.45$ (d, $J=6.9 \mathrm{~Hz}, 3 \mathrm{H}) .{ }^{13} \mathrm{C}-\mathrm{NMR}\left(75 \mathrm{MHz}, \mathrm{CHCl}_{3}\right) \delta 177.79,138.18,128.83$, 128.45, 126.43, 74.66, 71.27, 36.21, 18.16. IR (neat): 3027, 2990, 2938, 2872, 1716, 1115 $\mathrm{cm}^{-1}$. EI-LRMS, m/z (\%): $149\left(\mathrm{M}^{+}-\mathrm{CO}_{2} \mathrm{H}, 0.1\right), 122$ (10), 104 (100), 91 (79), 77 (63), 45 (67). ESI-HRMS: calculated for $\mathrm{C}_{11} \mathrm{H}_{14} \mathrm{O}_{3} \mathrm{Na}(\mathrm{M}+\mathrm{Na})^{+}: 217.0840$; found: 217.0833 .

Methyl methyl(2-phenethoxypropanoyloxy)carbamodithioate (25). Prepared according to

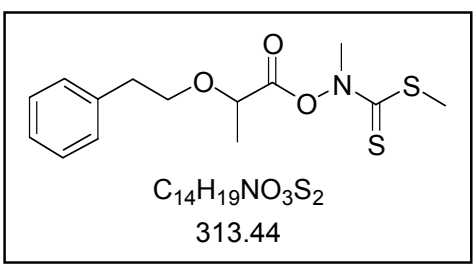

General procedure B from 2-(2-phenethoxy)propanoic acid (1.15 g, $5.90 \mathrm{mmol})$, methyl hydroxy(methyl)carbamodithioate (806 mg, $5.09 \mathrm{mmol})$, DCC (1.45 $\mathrm{mg}, 7.10 \mathrm{mmol})$ and DMAP (73 mg, $0.60 \mathrm{mmol})$ in $\mathrm{CH}_{2} \mathrm{Cl}_{2}(100 \mathrm{~mL})$. Purification by $\mathrm{FC}$ (cyclohexane/t-BuOMe 20:1) gave 25 (1.35 g, 73\%). Colorless oil. ${ }^{1} \mathrm{H}-\mathrm{NMR}(300 \mathrm{MHz}$, $\left.\mathrm{CDCl}_{3}\right) \delta$ 7.34-7.29 (m, 5H), 4.17 (dq, $\left.J=0.4,6.9 \mathrm{~Hz}, 1 \mathrm{H}\right), 3.95$ (ddt, $J=0.4,9.1,7.1 \mathrm{~Hz}$, 1H), 3.77 (s, 3H), 3.71 (ddt, $J=0.4,9.1,7.2 \mathrm{~Hz}, 1 \mathrm{H}), 2.95$ (t, $J=7.1 \mathrm{~Hz}, 2 \mathrm{H}), 2.55$ (s, 3H), $1.55(\mathrm{~d}, J=6.9 \mathrm{~Hz}, 3 \mathrm{H}) .{ }^{13} \mathrm{C}-\mathrm{NMR}\left(75 \mathrm{MHz}, \mathrm{CHCl}_{3}\right) \delta 196.95,169.62,138.17,128.87$, $128.35,126.35,73.70,71.69,42.64,36.17,18.69,18.45$. IR (neat): 2921, 2847, 1799, 1453, 1356, 1079, 1012, $698 \mathrm{~cm}^{-1}$. ESI-LRMS, $m / z(\%): 336\left(\mathrm{M}+\mathrm{Na}^{+}, 10\right), 314\left(\mathrm{M}+\mathrm{H}^{+}, 100\right), 266$ 
(8), 193 (7), 179 (4), 157 (6). ESI-HRMS: calculated for $\mathrm{C}_{14} \mathrm{H}_{19} \mathrm{O}_{3} \mathrm{NaS}_{2}(\mathrm{M}+\mathrm{Na})^{+}$: 336.0704 ; found: 336.0700 .

(2-(1-Azidoethoxy)ethyl)benzene (28). Prepared according to General Procedure E from 25

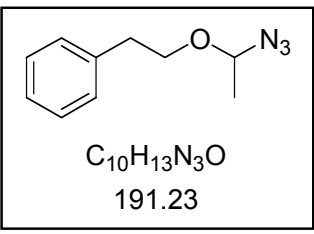
(100 mg, $0.32 \mathrm{mmol}), 3$-pyridinesulfonyl azide (354 mg, $1.91 \mathrm{mmol})$ and AIBN (260 mg, $1.60 \mathrm{mmol}$, added in 10 portions every $\mathrm{h}$ ) in benzene (12 mL). Purification by two FC (cyclohexane/t-BuOMe 10:1 and 15:1) gave 28 (43 mg, 70\%). Colorless oil. ${ }^{1} \mathrm{H}-\mathrm{NMR}\left(300 \mathrm{MHz}, \mathrm{CDCl}_{3}\right) \delta$ 7.29-7.15 (m, 5H), 4.53 (q, $J=5.6 \mathrm{~Hz}, 1 \mathrm{H}), 3.99(\mathrm{dt}, J=9.4,7.1 \mathrm{~Hz}, 1 \mathrm{H}), 3.68$ (dt, $J=9.4$, $7.2 \mathrm{~Hz}, 1 \mathrm{H}), 2.93(\mathrm{t}, J=7.2 \mathrm{~Hz}, 2 \mathrm{H}), 1.45(\mathrm{~d}, J=5.7 \mathrm{~Hz}, 3 \mathrm{H}) .{ }^{13} \mathrm{C}-\mathrm{NMR}\left(75 \mathrm{MHz}, \mathrm{CHCl}_{3}\right) \delta$ 138.32, 128.77, 128.29, 126.24, 89.09, 69.56, 35.99, 20.16. IR (neat): 2936, 2868, 2103, 1228, 1106, $697 \mathrm{~cm}^{-1}$. ESI-LRMS, $m / z(\%): 214\left(\mathrm{M}+\mathrm{Na}^{+}, 22\right), 189$ (50), 179 (10), 175 (36), 168 (43). ESI-HRMS: calculated for $\mathrm{C}_{10} \mathrm{H}_{13} \mathrm{~N}_{3} \mathrm{ONa}(\mathrm{M}+\mathrm{Na})^{+}:$: 214.0956; found: 214.0960.

2-(Cyclohex-3-enylmethoxy)ethanoic acid. 3-Cyclohexene-1-methanol $\overbrace{\substack{\mathrm{C}_{9} \mathrm{H}_{14} \mathrm{O}_{3} \\ 170.21}}^{\mathrm{O}} \mathrm{CO}_{2} \mathrm{H}$ $(2.20 \mathrm{~g}, 20.0 \mathrm{mmol})$ was added at $0{ }^{\circ} \mathrm{C}$ to a solution of $\mathrm{NaH}(1.80 \mathrm{~g}, 44$ mmol of a $60 \%$ dispersion in oil) in THF $(25 \mathrm{~mL})$. The resulting mixture was stirred for $30 \mathrm{~min}$ and 2-bromopropionic acid $(2.80 \mathrm{~g}, 20.0 \mathrm{mmol})$ was added slowly at $0{ }^{\circ} \mathrm{C}$ and the reaction mixture was heated under reflux for $4 \mathrm{~h}$. After cooling down to $\mathrm{rt}, \mathrm{H}_{2} \mathrm{O}$ and $t$-BuOMe were added. The resulting mixture was extracted with sat. $\mathrm{NaHCO}_{3}$. The aqueous extract was carefully acidified with $\mathrm{HCl} 4 \mathrm{M}$ extracted with $t$-BuOMe. Drying with $\mathrm{MgSO}_{4}$ and concentration under reduced pressure gave 2-(cyclohex-3-enylmethoxy)ethanoic acid (3.25 g, 95\%). Colorless oil. ${ }^{1} \mathrm{H}-\mathrm{NMR}(300 \mathrm{MHz}$, $\left.\mathrm{CDCl}_{3}\right) \delta 11.43$ (br s, 1H), 5.63-5.57 (m, 2H), 4.09 (s, 2H), 3.39 (d, $\left.J=6.6 \mathrm{~Hz}, 2 \mathrm{H}\right), 2.10-1.64$ $(\mathrm{m}, 6 \mathrm{H}), 1.30-1.15(\mathrm{~m}, 1 \mathrm{H}) .{ }^{13} \mathrm{C}-\mathrm{NMR}\left(75 \mathrm{MHz}, \mathrm{CHCl}_{3}\right) \delta$ 175.57, 126.85, 125.53, 76.60, 67.74, 33.49, 28.08, 25.19, 24.22. IR (neat): 3022, 2913, 2839, 1724, 1436, 1135, $653 \mathrm{~cm}^{-1}$. ESI-LRMS, m/z (\%): $193\left(\mathrm{M}+\mathrm{Na}^{+}, 19\right), 149$ (14), 123 (18), 118 (27), 102 (100). HRMS: Determination of the exact mass was not possible due to the instability of the azide group.

Methyl 2-(cyclohex-3-enylmethoxy)ethanoyloxy(methyl)carbamodithioate (26). Prepared $\overbrace{\substack{\mathrm{C}_{12} \mathrm{H}_{19} \mathrm{NO}_{3} \mathrm{~S}_{2} \\ 289.42}}^{\mathrm{O}_{\mathrm{N}^{2}}} \mathrm{~N}_{\mathrm{O}^{-}}^{\mathrm{S}}$ according to General Procedure B from 2-(cyclohex-3enylmethoxy)ethanoic acid (1.00 g, $5.90 \mathrm{mmol})$, methyl 
hydroxy(methyl)carbamodithioate $(806 \mathrm{mg}, 5.90 \mathrm{mmol})$, DCC (1.45 g, $7.10 \mathrm{mmol})$ and DMAP (73 mg, $0.60 \mathrm{mmol})$ in $\mathrm{CH}_{2} \mathrm{Cl}_{2}(100 \mathrm{~mL})$. Purification by FC (cyclohexane/t-BuOMe 10:1) gave 26 (1.31 g, 76\%). Colorless oil. ${ }^{1} \mathrm{H}-\mathrm{NMR}\left(300 \mathrm{MHz}, \mathrm{CDCl}_{3}\right) \delta 5.62-5.54(\mathrm{~m}, 2 \mathrm{H})$, 4.25 (s, 2H), 3.73 (s, 3H), 3.43 (d, $J=6.4 \mathrm{~Hz}, 2 \mathrm{H}), 2.47$ (s, 3H), 2.08-1.65 (m, 6H), 1.31-1.18 (m, 2H). ${ }^{13} \mathrm{C}-\mathrm{NMR}\left(75 \mathrm{MHz}, \mathrm{CHCl}_{3}\right) \delta$ 196.60, 167.03, 126.67, 125.38, 76.81, 66.29, 42.39, 33.49, 27.88, 25.00, 24.10, 18.35. IR (neat): 3022, 2917, 2834, 1811, 1423, 1356, 1056, 1004 $\mathrm{cm}^{-1}$. ESI-LRMS, m/z (\%): $312\left(\mathrm{M}^{+}+\mathrm{Na}, 68\right), 239$ (23), 215 (79), 133 (23). ESI-HRMS: calculated for $\mathrm{C}_{12} \mathrm{H}_{19} \mathrm{NO}_{3} \mathrm{NaS}_{2}(\mathrm{M}+\mathrm{Na})^{+}:$312.0704; found: 312.0698 .

(Cyclohex-3-enemethoxy)azidomethane (29). Prepared according to General Procedure E

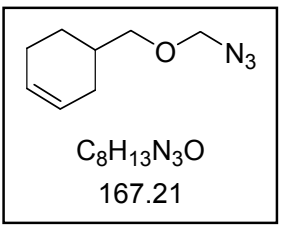
from 26 (166 mg, $0.57 \mathrm{mmol}), \mathrm{PhSO}_{2} \mathrm{~N}_{3}(630 \mathrm{mg}, 3.44 \mathrm{mmol})$ and AIBN (416 mg, $2.57 \mathrm{mmol}$, added in 9 portions every $\mathrm{h}$ ) in benzene $(20 \mathrm{~mL})$. Two purifications by FC (cyclohexane/t-BuOMe 30:1 and 80:1) gave 28 (60 mg, 63\%). Colorless oil. ${ }^{1} \mathrm{H}-\mathrm{NMR}\left(300 \mathrm{MHz}, \mathrm{CDCl}_{3}\right) \delta 5.70-5.61(\mathrm{~m}, 2 \mathrm{H})$, $4.64(\mathrm{~s}, 2 \mathrm{H}), 3.49$ (d, $J=6.6 \mathrm{~Hz}, 2 \mathrm{H}), 2.16-1.67(\mathrm{~m}, 6 \mathrm{H}), 1.37-1.24(\mathrm{~m}, 1 \mathrm{H}) .{ }^{13} \mathrm{C}-\mathrm{NMR}(75$ $\left.\mathrm{MHz}, \mathrm{CHCl}_{3}\right) \delta 126.96,125.62,82.97,74.48,33.73,28.24,25.37,24.37$. IR (neat): 2914, 2893, 2116, 1225, 1110, 1067, 872, $653 \mathrm{~cm}^{-1}$. EI-LRMS, $m / z(\%): 125\left({\mathrm{M}-\mathrm{N}_{3}}^{+}, 27\right), 111(32)$, 93 (88), 79 (100), 67 (85), 53 (55), 41 (78). HRMS: Determination of the exact mass was not possible due to the instability of the azide group.

Dimethyl 2-(3-methylbut-2-enyl)propanedioate. Dimethyl malonate (132 mg, $1 \mathrm{mmol})$ was \begin{tabular}{c|}
$\mathrm{MeOOC}$ \\
$\mathrm{MeOOC}$ \\
$\mathrm{C}_{10} \mathrm{H}_{16} \mathrm{O}_{4}$ \\
200.23 \\
\hline
\end{tabular} added at $0{ }^{\circ} \mathrm{C}$ to a suspension of $\mathrm{NaH}(43 \mathrm{mg}, 1.1 \mathrm{mmol}$ of a $60 \%$ dispersion in oil) in THF $(20 \mathrm{~mL})$. The reaction mixture was stirred at $0{ }^{\circ} \mathrm{C}$ for $30 \mathrm{~min}$ and 1-bromo-3-methylbut-2-ene (149 $\mathrm{mg}, 1.00 \mathrm{mmol})$ was added at $0{ }^{\circ} \mathrm{C}$ to the clear solution. The mixture was stirred overnight at $\mathrm{rt}$, $\mathrm{H}_{2} \mathrm{O}$ was slowly added and the resulting biphasic mixture was extracted with EtOAc. The combined organic extracts were dried with $\mathrm{MgSO}_{4}$ and concentrated. Purification of the residue by FC (hexane/EtOAc 20:1) gave dimethyl 2-(3-methylbut-2enyl)propanedioate (94 mg, 47\%). Colorless oil. Physical and spectral data were in accordance with literature data. ${ }^{[8,9]}{ }^{1} \mathrm{H}-\mathrm{NMR}\left(300 \mathrm{MHz}, \mathrm{CDCl}_{3}\right) \delta$ 5.07-5.01 (m, 1H), $3.72(\mathrm{~s}$, $6 \mathrm{H}), 3.36(\mathrm{t}, 1 \mathrm{H}), 2.58(\mathrm{t}, 2 \mathrm{H}), 1.67(\mathrm{~s}, 3 \mathrm{H}), 1.62(\mathrm{~s}, 3 \mathrm{H}) \cdot{ }^{13} \mathrm{C}-\mathrm{NMR}\left(75 \mathrm{MHz}, \mathrm{CDCl}_{3}\right) \delta$ $169.46,169.45,134.92,119.39,52.26,51.77,27.50,17.54$. 
1-tert-Butyl 3,3-dimethyl 6-methylhept-5-ene-1,3,3-tricarboxylate. Dimethyl 2-(3-

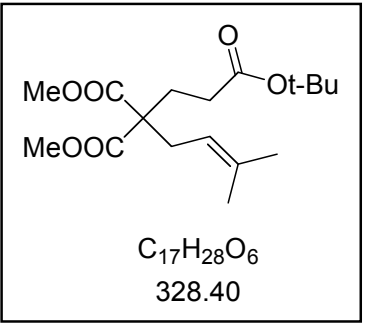
methylbut-2-enyl)propanedioate (94 $\mathrm{mg}, 0.47 \mathrm{mmol})$ in THF $(2 \mathrm{~mL})$ was added at $0{ }^{\circ} \mathrm{C}$ to suspension of $\mathrm{NaH}(20 \mathrm{mg}, 0.52 \mathrm{mmol}$ of a $60 \%$ dispersion in oil) in THF (10 mL). The mixture was stirred 30 min at 0 ${ }^{\circ} \mathrm{C}$ and $t$-butyl acrylate $(60 \mathrm{mg}, 0.47 \mathrm{mmol})$ was added at $0{ }^{\circ} \mathrm{C}$ to the clear solution. The resulting mixture was stirred overnight at $\mathrm{rt}$ and $\mathrm{H}_{2} \mathrm{O}$ was cautiously added. The reaction mixture was extracted with EtOAc and the combined organic extracts were dried with $\mathrm{MgSO}_{4}$ and concentrated under vacuum. Purification of the residue by FC (hexane/EtOAc 15:1) gave the desired triester ( $58 \mathrm{mg}, 38 \%$ ). Colorless oil. ${ }^{1} \mathrm{H}$ NMR (300 MHz, $\left.\mathrm{CDCl}_{3}\right) \delta$ 4.96-4.90 (m, 1H), $3.68(\mathrm{~s}, 6 \mathrm{H}), 2.57(\mathrm{~d}, J=7.5 \mathrm{~Hz}, 2 \mathrm{H}), 2.21-$ $2.08(\mathrm{~m}, 4 \mathrm{H}), 1.66(\mathrm{~s}, 3 \mathrm{H}), 1.59(\mathrm{~s}, 3 \mathrm{H}), 1.41(\mathrm{~s}, 9 \mathrm{H}) .{ }^{13} \mathrm{C}-\mathrm{NMR}\left(75 \mathrm{MHz}, \mathrm{CDCl}_{3}\right) \delta$ 172.04, $171.58,135.90,117.25,80.43,56.99,52.33,31.88,30.68,28.01,27.66,25.95,17.80$. IR (neat): 2978, 2952, 2931, 1728, 1436, 1367, 1216, 1149, 1080, $846 \mathrm{~cm}^{-1}$. EI-LRMS, $m / z(\%)$ : $328\left(\mathrm{M}^{+}, 2\right), 272$ (32), 255 (40), 212 (36), 204 (50), 173 (55), 162 (41), 69 (68), 57 (100), 41 (90). ESI-HRMS: calculated for $\mathrm{C}_{17} \mathrm{H}_{28} \mathrm{O}_{6} \mathrm{Na}$ : 351.1783; found: 351.1771 .

4,4-Bis(methoxycarbonyl)-7-methyloct-6-enoic acid. 1-tert-Butyl 3,3-dimethyl 6-

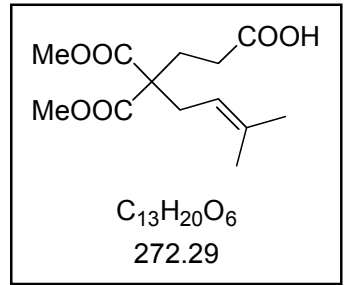
methylhept-5-ene-1,3,3-tricarboxylate $(1.2 \mathrm{~g}, 3.65 \mathrm{mmol})$ and $p$ toluenesulfonic acid (139 $\mathrm{mg}, 0.73 \mathrm{mmol})$ were dissolved in toluene $(90 \mathrm{~mL})$ and the solution was stirred at $100{ }^{\circ} \mathrm{C}$ for $2 \mathrm{~h}$. Concentration under reduced pressure and purification by $\mathrm{FC}$ (hexane/EtOAc 2:1) gave the monocarboxylic acid (474 mg, 48\%). Colorless oil. ${ }^{1} \mathrm{H}-\mathrm{NMR}$ $\left(300 \mathrm{MHz}, \mathrm{CDCl}_{3}\right) \delta$ 4.96-4.90 (m, 1H), $3.70(\mathrm{~s}, 6 \mathrm{H}), 2.60$ (d, $\left.J=7.5 \mathrm{~Hz}, 2 \mathrm{H}\right), 2.39-2.35$ (m, 2H), 2.20-2.15 (m, 2H), $1.68(\mathrm{~s}, 3 \mathrm{H}), 1.60(\mathrm{~s}, 3 \mathrm{H}) .{ }^{13} \mathrm{C}-\mathrm{NMR}\left(75 \mathrm{MHz}, \mathrm{CHCl}_{3}\right) \delta$ 178.90, $171.41,136.15,116.99,56.80,52.39,31.99,29.34,27.29,25.91,17.78$. IR (neat): 2953, 2359, 2336, 1729, 1714, 1435, 1218, 1173, 1079, 911, $729 \mathrm{~cm}^{-1}$. EI-LRMS, $m / z(\%): 272\left(\mathrm{M}^{+}\right.$, 6), 255 (7), 241 (12), 212 (20), 204 (30), 195 (24), 162 (57), 135 (34), 113 (42), 93 (37), 69 (100), 59 (56), 41 (57). EI-HRMS: calculated for $\mathrm{C}_{13} \mathrm{H}_{20} \mathrm{O}_{6}$ : 272.1260; found: 272.1260.

Dimethyl 2-(3-methylbut-2-enyl)-2-(3-[(methylthiocarbonothioyl)(phenyl)aminooxy)-3oxopropyl]propanedioate (21). Prepared according to General

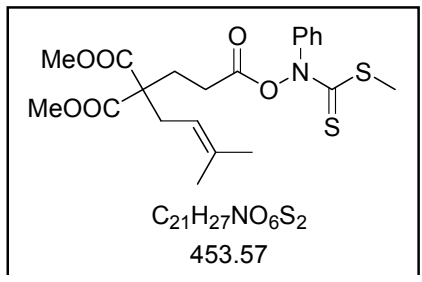


Procedure B from 4,4-bis(methoxycarbonyl)-7-methyloct-6-enoic acid (460 mg, $1.69 \mathrm{mmol}$ ), DCC (384 mg, $1.86 \mathrm{mmol})$, DMAP $(20 \mathrm{mg}, 0.17 \mathrm{mmol})$ and methyl hydroxy(phenyl)carbamodithioate $(337 \mathrm{mg}, 1.69 \mathrm{mmol})$ in $\mathrm{CH}_{2} \mathrm{Cl}_{2}(30 \mathrm{~mL})$. Purification by FC (hexane/EtOAc 7:1) gave 21 (425 mg, 55\%). Yellowish oil. ${ }^{1} \mathrm{H}-\mathrm{NMR}\left(300 \mathrm{MHz}, \mathrm{CDCl}_{3}\right.$ ) ठ 7.57-7.42 (m, 5H), 4.95-4.89 (m, 1H), $3.67(\mathrm{~s}, 6 \mathrm{H}), 2.61-2.49(\mathrm{~m}, 4 \mathrm{H}), 2.60(\mathrm{~s}, 3 \mathrm{H}), 2.26-$ $2.20(\mathrm{~m}, 2 \mathrm{H}), 1.68(\mathrm{~d}, J=1.68 \mathrm{~Hz}, 3 \mathrm{H}), 1.60(\mathrm{~d}, J=1.60 \mathrm{~Hz}, 3 \mathrm{H}) .{ }^{13} \mathrm{C}-\mathrm{NMR}(75 \mathrm{MHz}$, $\left.\mathrm{CHCl}_{3}\right) \delta 197.49,171.09,169.32,141.04,136.33,129.93,129.28,128.19,116.82,56.70$, 52.40, 32.23, 27.01, 26.80, 25.90, 19.17, 17.79. IR (neat): 2976, 2864, 1729, 1229, 1148, 844 $\mathrm{cm}^{-1}$. ESI-LRMS, m/z (\%): $476\left(\mathrm{M}+\mathrm{Na}^{+}, 40\right), 381$ (19), 182 (8), 149 (3), 132 (100). ESIHRMS: calculated for $\mathrm{C}_{21} \mathrm{H}_{27} \mathrm{NO}_{6} \mathrm{~S}_{2} \mathrm{Na}(\mathrm{M}+\mathrm{Na})^{+}$: 476.1177 ; found: 476.1186 .

\section{Dimethyl 3-(2-(methylthio(phenylimino)methylthio)propan-2-yl)cyclopentane-1,1-} dicarboxylate (22). Prepared according to General Procedure C

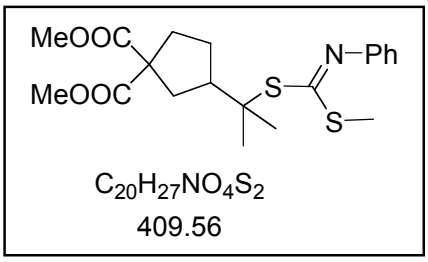
from 21 (104 mg, $0.23 \mathrm{mmol})$ in $\mathrm{CH}_{2} \mathrm{Cl}_{2}(7 \mathrm{~mL})$. Purification by FC (cyclohexane/t-BuOMe 10:1) gave 22 (75 mg, 80\%). ${ }^{1} \mathrm{H}-\mathrm{NMR}$ $\left(300 \mathrm{MHz}, \mathrm{CDCl}_{3}\right) \delta$ 7.36-7.27 (m, 2H), 7.09-7.03 (m, 1H), 6.86$6.83(\mathrm{~m}, 2 \mathrm{H}), 3.71(\mathrm{~s}, 6 \mathrm{H}), 2.70(\mathrm{br} \mathrm{s}, 1 \mathrm{H}), 2.39(\mathrm{~s}, 3 \mathrm{H}), 2.49-1.98$ (m, 4H), 1.76 (br s, 1H), 1.63-1.46 (m, 7H). ${ }^{13} \mathrm{C}-\mathrm{NMR}\left(75 \mathrm{MHz}, \mathrm{CHCl}_{3}\right) \delta 172.65,159.50$, $149.64,128.78,123.67,120.43,59.82$, 55.71 52.67, 52.65, 48.42, 35.90, 33.87, 26.90, 25.99, 16.07. IR (neat): 2952, 1730, 1569, 1255, 1201, 1163, 913, $729 \mathrm{~cm}^{-1}$. EI-LRMS, $m / z(\%): 409$ $\left(\mathrm{M}^{+}, 0.3\right), 362$ (1), 274 (4), 184 (36), 167 (71), 150 (38), 135 (51), 107 (100), 84 (80). ESIHRMS: calculated for $\mathrm{C}_{20} \mathrm{H}_{27} \mathrm{NO}_{4} \mathrm{~S}_{2}$ : 410.1459; found: 410.1463 .

Dimethyl 3-(2-azidopropan-2-yl)cyclopentane-1,1-dicarboxylate (23). Prepared according

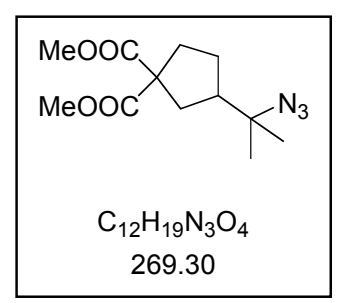
to General Procedure E from 21 (200 $\mathrm{mg}, 0.44 \mathrm{mmol})$ and benzenesulfonyl azide (485 $\mathrm{mg}, 2.65 \mathrm{mmol})$ in $\mathrm{CH}_{2} \mathrm{Cl}_{2}(20 \mathrm{~mL})$. Purification by FC (hexane/EtOAc 20:1) gave a mixture of 23 (49 mg, 41\%) contaminated with 22 (14 $\mathrm{mg}, 8 \%$ ). A pure sample of 23 was obtained after a second FC (hexane/EtOAc 30:1). ${ }^{1} \mathrm{H}-\mathrm{NMR}(300 \mathrm{MHz}$, $\left.\mathrm{CDCl}_{3}\right) \delta 3.72(\mathrm{~s}, 6 \mathrm{H}), 2.41-2.25(\mathrm{~m}, 2 \mathrm{H}), 2.14-2.05(\mathrm{~m}, 2 \mathrm{H}), 1.99-1.92(\mathrm{~m}, 1 \mathrm{H}), 1.79-1.71$ $(\mathrm{m}, 1 \mathrm{H}), 1.63-1.53(\mathrm{~m}, 1 \mathrm{H}), 1.27(\mathrm{~s}, 6 \mathrm{H}) .{ }^{13} \mathrm{C}-\mathrm{NMR}\left(75 \mathrm{MHz}, \mathrm{CHCl}_{3}\right) \delta 172.81,172.52$, $62.70,59.83,52.72,52.68,49.13,35.63,33.66,26.63,24.80$. IR (neat): 2954, 2100, 1730, 


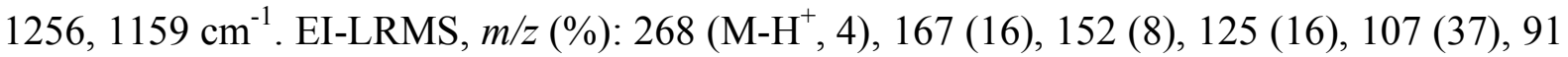
(100), 82 (34), 56 (85), 41 (55). HRMS: Determination of the exact mass was not possible due to the instability of the azide group.

1-(Naphthalen-2-yl)propane-2-thiol (19). A solution of 14 (200 mg, $0.56 \mathrm{mmol})$ in THF (3

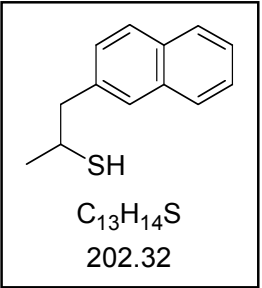
$\mathrm{mL})$ was added over a period of $5 \mathrm{~min}$ at $0{ }^{\circ} \mathrm{C}$ to a solution of $\mathrm{LiAlH}_{4}(107$ $\mathrm{mg}, 2.81 \mathrm{mmol})$ in THF $(22 \mathrm{~mL})$. The reaction mixture was heated under reflux for $3 \mathrm{~h}$, cooled down to rt and poured into EtOAc. The layers were separated, the organic layer was washed with $\mathrm{H}_{2} \mathrm{O}$, dried with $\mathrm{MgSO}_{4}$ and concentrated under reduced pressure. Purification by FC (cyclohexane/tBuOMe 50:1) gave 19 (85 mg, 76\%). Colorless oil. Physical and spectral data were in accordance with literature data. ${ }^{[10]}{ }^{1} \mathrm{H}-\mathrm{NMR}\left(300 \mathrm{MHz}, \mathrm{CDCl}_{3}\right) \delta$ 7.83-7.78 (m, 3H), $7.64(\mathrm{~s}$, $1 \mathrm{H}), 7.49-7.42(\mathrm{~m}, 2 \mathrm{H}), 7.34-7.31(\mathrm{~m}, 1 \mathrm{H}), 3.38-3.30(\mathrm{~m}, 1 \mathrm{H}), 3.08-2.95(\mathrm{~m}, 2 \mathrm{H}), 1.38(\mathrm{~d}$, 3H). ${ }^{13} \mathrm{C}-\mathrm{NMR}\left(75 \mathrm{MHz}, \mathrm{CHCl}_{3}\right) \delta 136.82,133.40,132.25,127.97,127.70,127.60,127.55$, 127.47, 126.01, 125.46, 47.51, 36.59, 24.38. IR (neat): 3054, 2955, 2920, 2860, 2547, 2361, 2339, 1596, 1505, 1446, 1370, 1271, 824, $746 \mathrm{~cm}^{-1}$. EI-LRMS, $m / z(\%): 202\left(\mathrm{M}^{+}, 62\right), 169$ (5), 141 (100), 115 (27), 75 (82), 61 (16). EI-HRMS: calculated for $\mathrm{C}_{13} \mathrm{H}_{14} \mathrm{~S}$ : 202.0816, found: 202.0817

4-Bromo-1-tosylpiperidine (20). A solution of $9(540 \mathrm{mg}, 1.16 \mathrm{mmol})$ in $\mathrm{CH}_{2} \mathrm{Cl}_{2}(10 \mathrm{~mL})$ $\mathrm{C}_{12} \mathrm{H}_{16} \mathrm{BrNO}_{2} \mathrm{~S}$
318.23 was slowly added over a period of $4 \mathrm{~h}$ to a solution of $\mathrm{BrCCl}_{3}(691 \mathrm{mg}, 3.49)$ in $\mathrm{CH}_{2} \mathrm{Cl}_{2}(20 \mathrm{~mL})$ at $5{ }^{\circ} \mathrm{C}$ under irradiation with a $300 \mathrm{~W}$ sun lamp. Upon completion of the reaction, the solvent was removed under reduced pressure and the crude product was purified by $\mathrm{FC}$ (cyclohexane/ $\mathrm{CH}_{2} \mathrm{Cl}_{2} 6: 1$ to $2: 1$ ) to afford 20 (299 mg, 86\%). White solid. Physical and spectral data were in accordance with literature data. ${ }^{[5]}{ }^{1} \mathrm{H}-\mathrm{NMR}\left(300 \mathrm{MHz}, \mathrm{CDCl}_{3}\right) \delta 7.58(\mathrm{~d}, J=8.3 \mathrm{~Hz}, 2 \mathrm{H}), 7.26(\mathrm{~d}, J=7.9 \mathrm{~Hz}$, 2H), 4.20-4.13 (m, 1H), 3.16-3.08 (m, 2H), 3.05-2.98 (m, 2H), 2.37 (s, 3H), 2.17-2.07 (m, 2H), 2.02-1.92 (m, 2H). ${ }^{13} \mathrm{C}-\mathrm{NMR}\left(75 \mathrm{MHz}, \mathrm{CHCl}_{3}\right) \delta$ 129.78, 127.60, 47.83, 43.82, 34.73, 21.55 
[1] S. Kim, C. J. Lim, S.-E. Song, H.-Y. Kang, Synlett 2001, 5, 688.

[2] F. G. Bordwell, W.-Z. Liu, J. Am. Chem. Soc. 1996, 118, 8777.

[3] E. Quast, Justus Liebigs Ann. Chem. 1974, 1727.

[4] P. Wessig, O. Muehling, Helv. Chim. Acta 2003, 86.

[5] C. Ollivier, P. Renaud, J. Am. Chem. Soc. 2001, 123, 4717.

[6] M. K. J. t. Wiel, R. A. v. Delden, A. Meetsma, B. L. Feringa, J. Am. Chem. Soc. 2003, $125,15076$.

[7] T. Nagasaka, H. Yamamoto, A. Sugiyama, F. Hamaguchi, Heterocycles 1988, 27, 2219.

[8] Y. I. M. Nilsson, P. G. Andersson, J.-E. Bäckvall, J. Am. Chem. Soc. 1993, 115, 6609.

[9] A. Padwa, M. A. Filipkowski, M. Meske, S. S. Murphree, S. H. Watterson, Z. Ni, J. Org. Chem. 1994, 59, 588.

[10] A. Marei, A. Sammour, T. Kassem, J. Chem. U. A. R. 1969, 12, 323. 


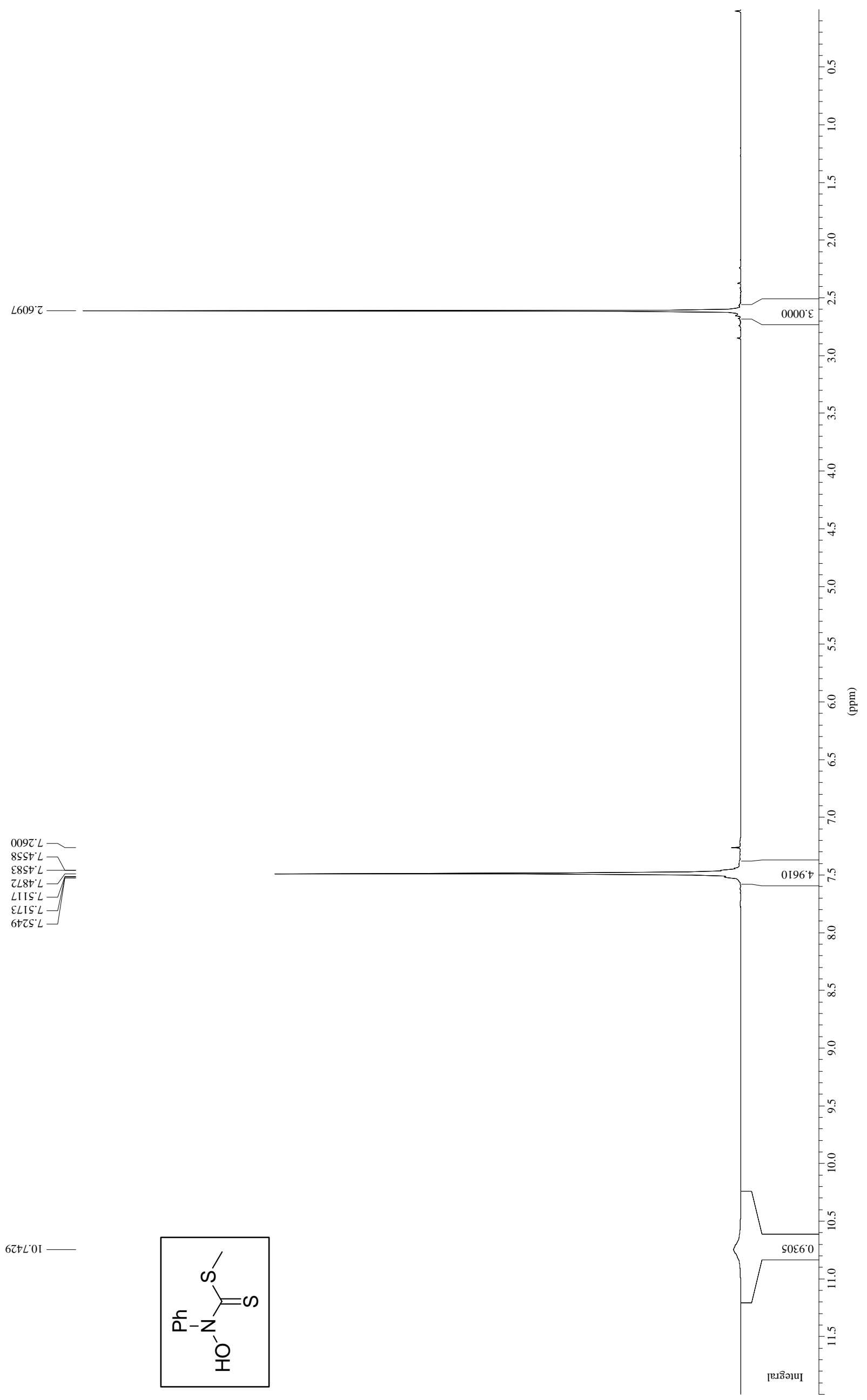


E8LS"9L

$0000^{\circ} \angle L$
$\angle I Z T^{\circ} \angle L$

$\angle S \angle S^{\circ} 9 Z$

9S6E* $6 Z \mathrm{I}$

$99 t \varepsilon^{\circ} 0 \varepsilon \mathrm{C}$

IIL6'LEI

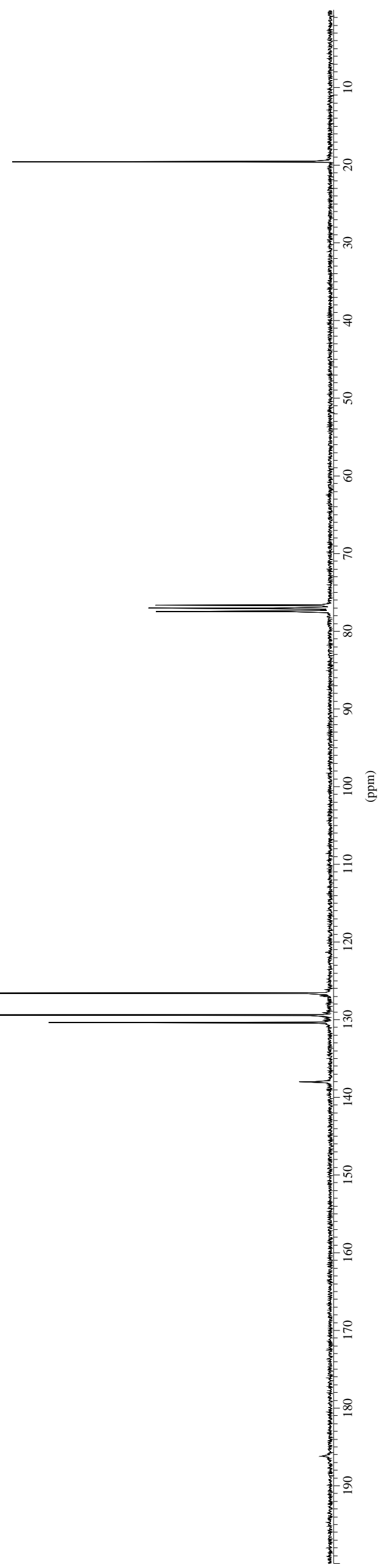




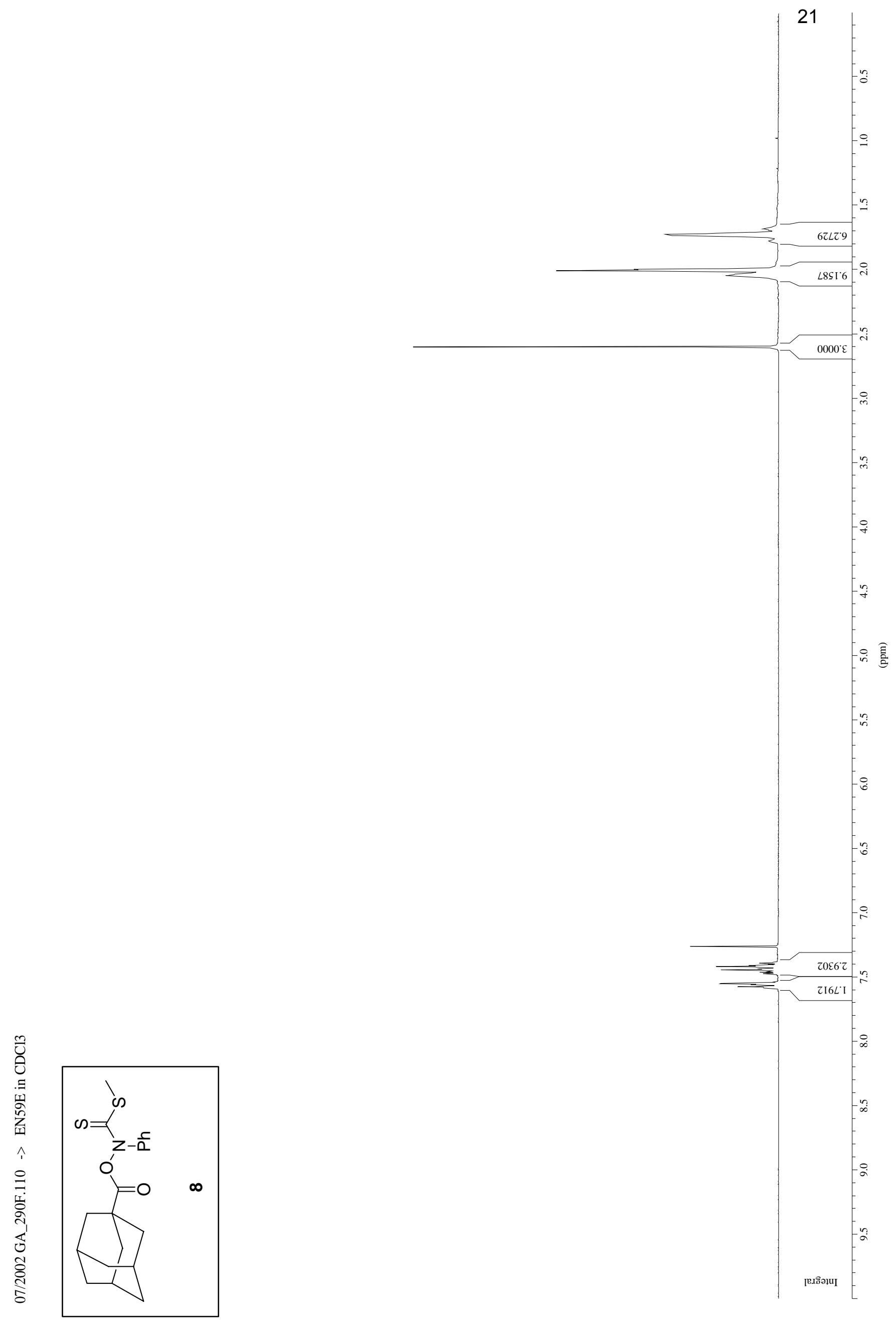




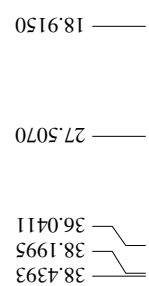

E8LS:9L $0000 \angle L$

LIZt'LL

I I09 LZI

$\angle 866^{\circ}{ }^{\circ} \mathrm{II}$
S6ZE

I8LE"It I
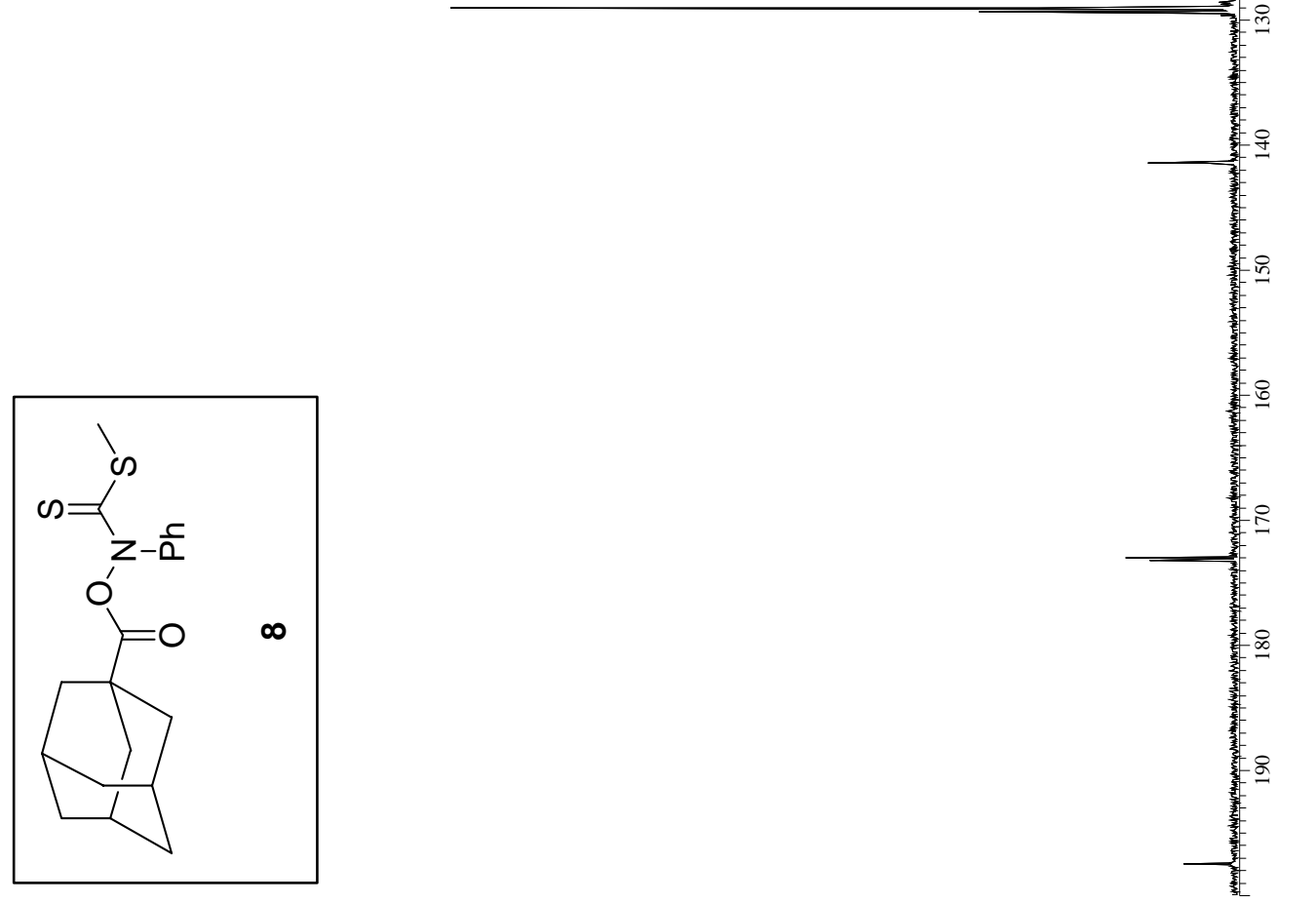


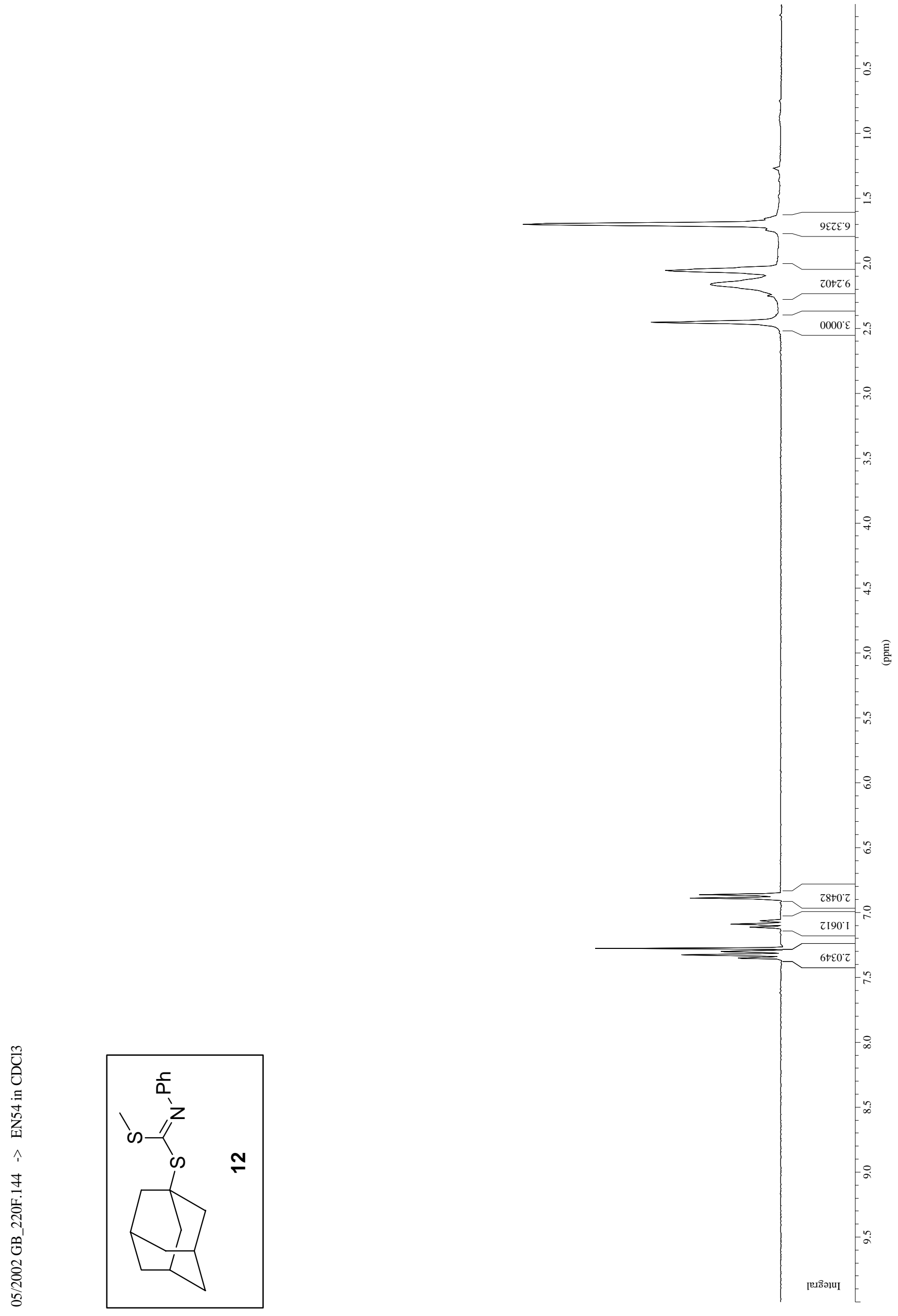


I966 SI

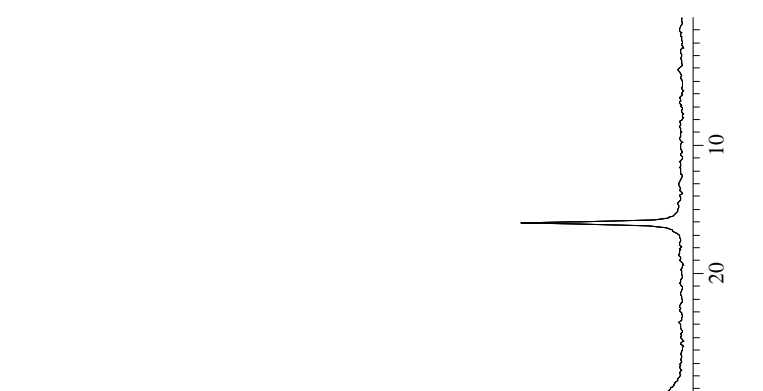

t9 $\angle 0^{\circ} 0 \varepsilon$

9T0เ 98

ง9เ8:Z๖

8858'25

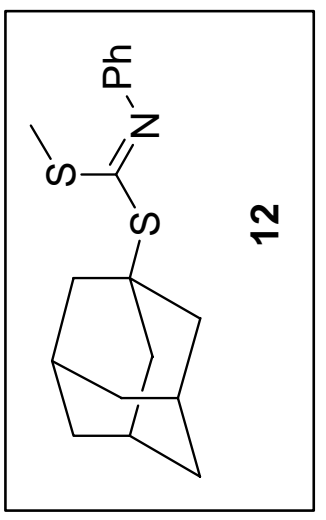




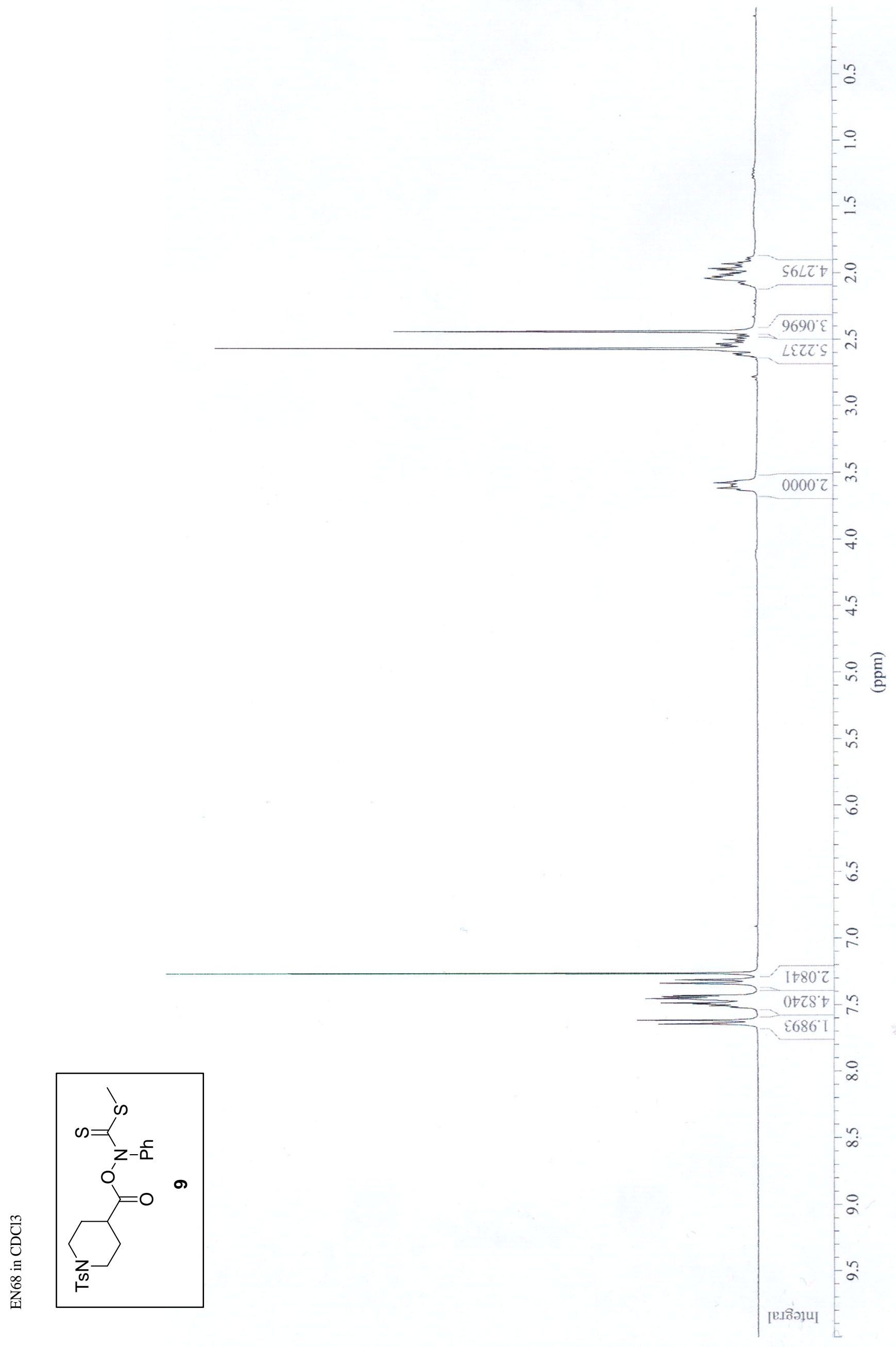



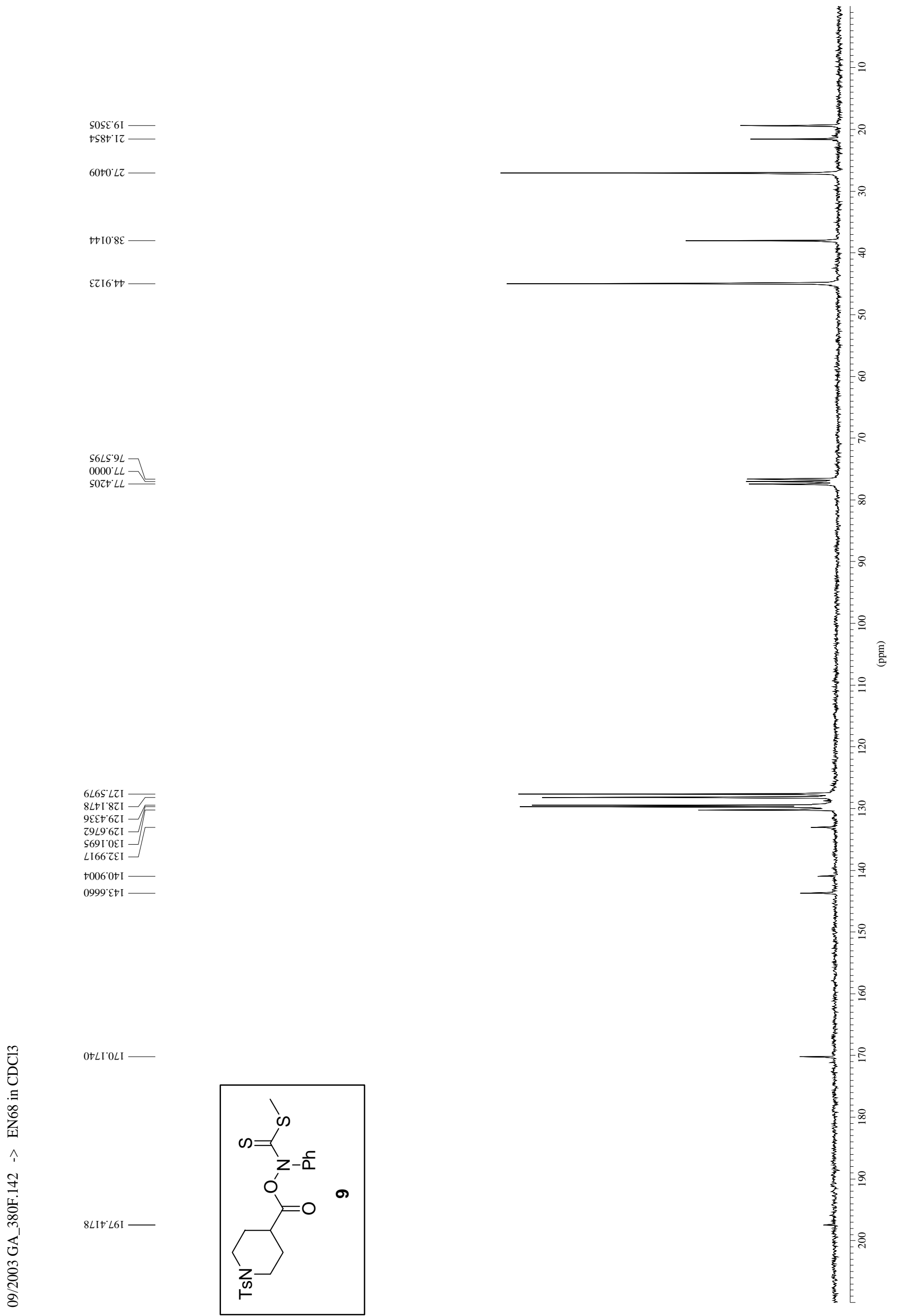

S69" 0 \&I

$\angle$ I66'ZEI

†006 0 t I

0999 E๋I 


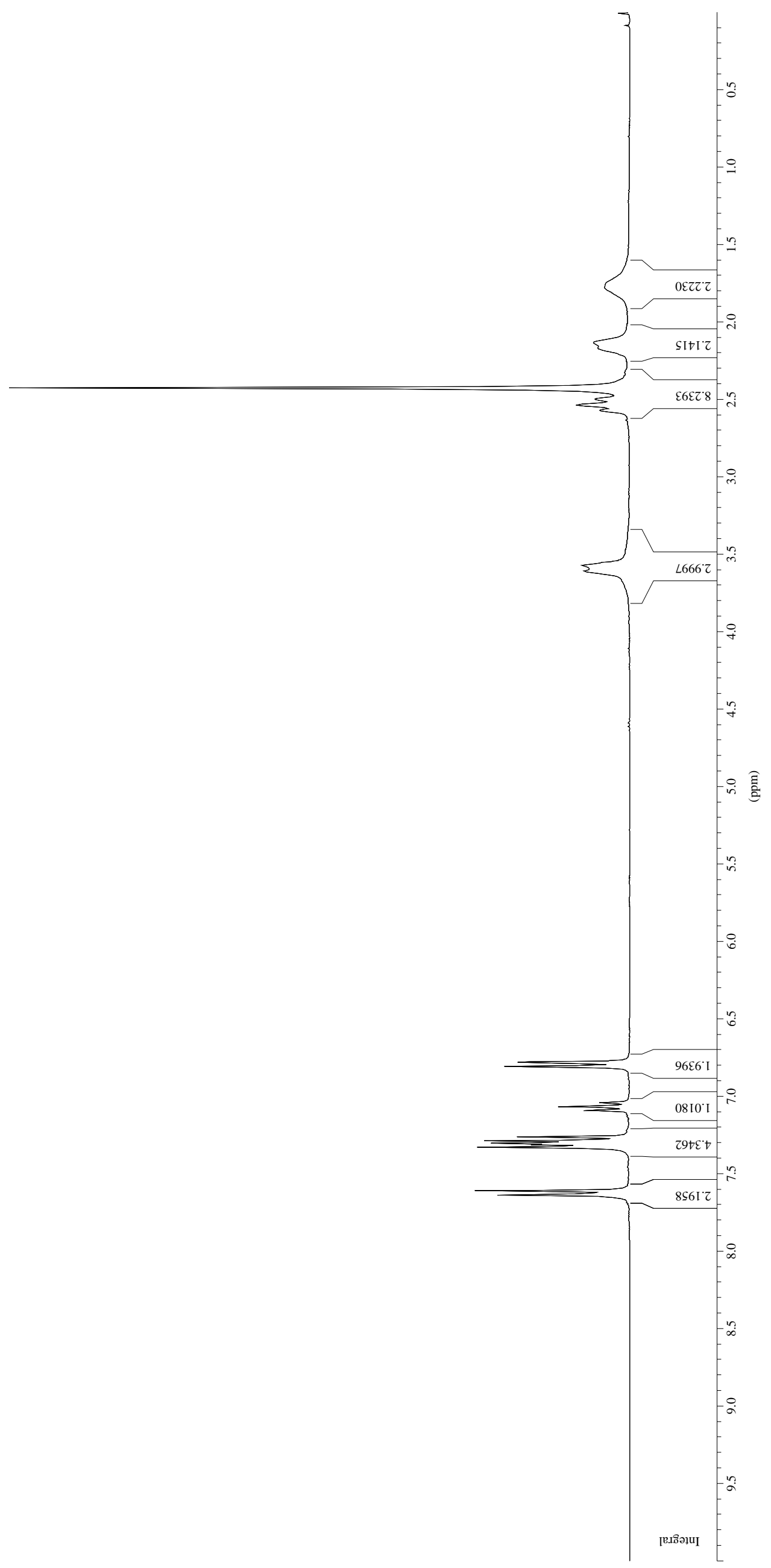


t9เ8

It0t' IZ ิ

OLIE. IE

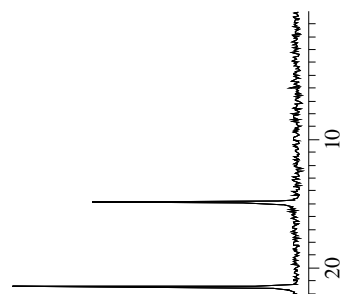

$\angle S \angle Z^{*} I t$

$\angle t C L^{\circ} \mathrm{S} t$

E8LS"9L

$0000 \angle L$

$\angle I Z \nabla^{\circ} \angle L$

E9Z0॰0ZI

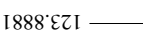

$z \& \oplus C^{\circ} \angle Z \mathrm{I}$

I8S8.8ZI

t65'6ZI

I LL8'ZEI

Z8ZC" $\mathrm{EtI}$

I $\angle 0 S^{\circ} 6 \mathrm{~T} \mathrm{I}$

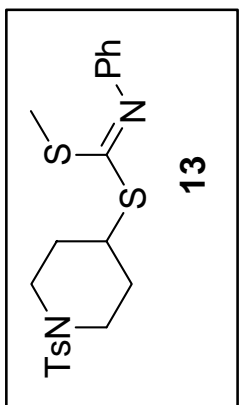




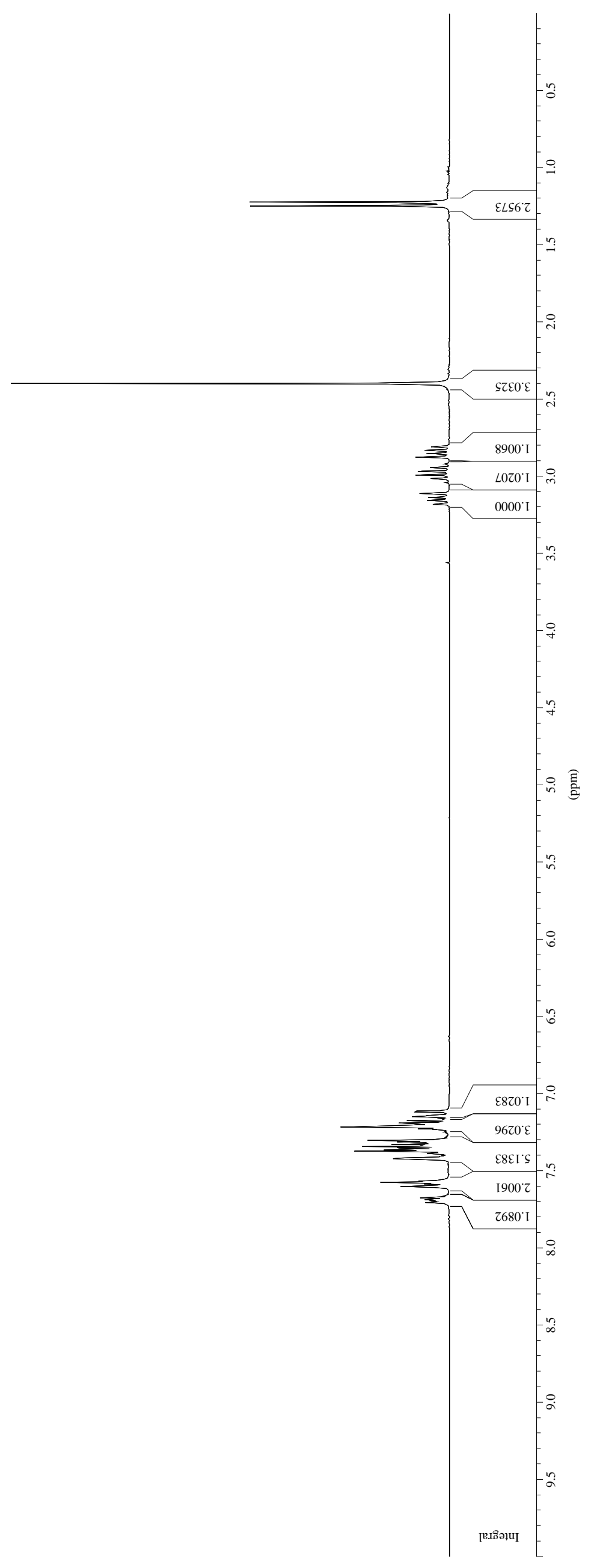


6EZL'9I -

IES0 6 I

S8z๕:6E

†6LS 94

$0000^{\circ} L L$
S0ZT $L L$

St8t"SZI

ZIZ6.SZI

SESO $\angle Z I$
Z06t $\angle Z I$

Z06t" $\angle Z I$
SZZS $^{\circ} \angle Z I$

ES6S LZI

9S08 LZI

Z850 $82 \mathrm{TI}$

†E80"6ZT

ES79.6ZI

608I'ZEI

乙EIE $E$ เ

Z0tt SEI

I690. It I

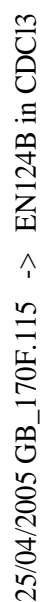
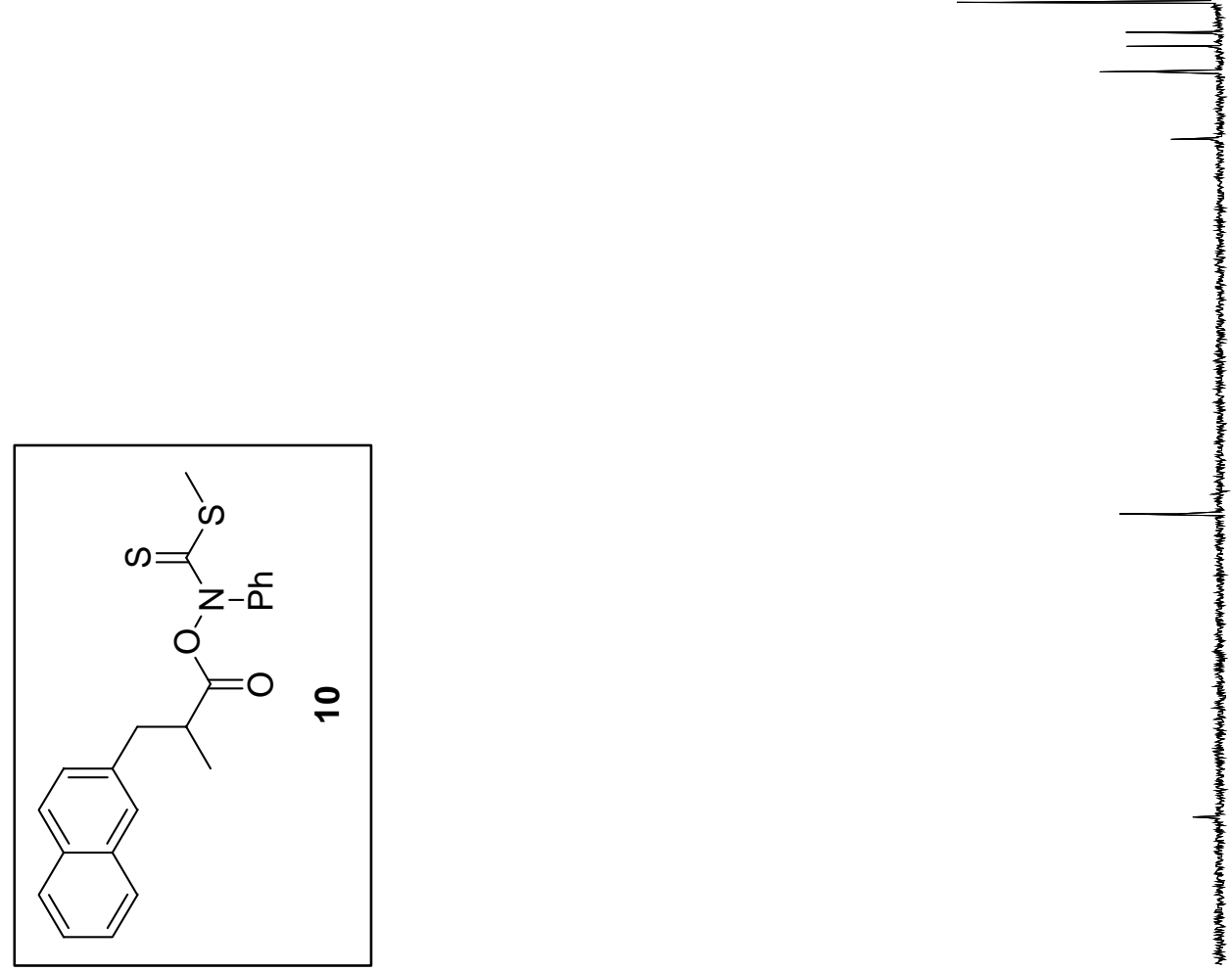

ESEE`ZLI

$\angle \angle 69^{\circ} \angle 6 \mathrm{I}$ 


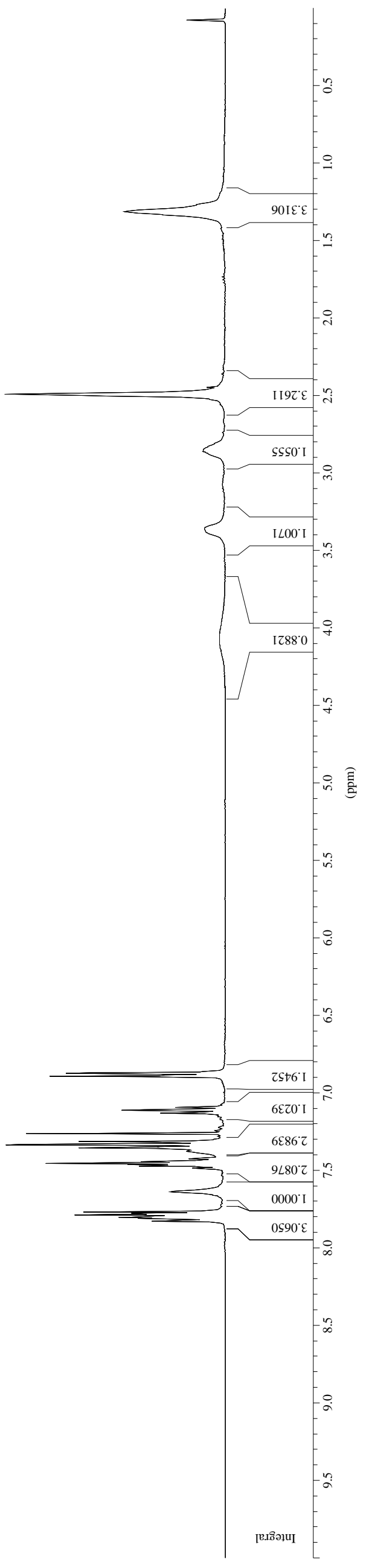


$8 t t 0^{\circ} S I$

ع9I8.6I -

โ869' $Z t$

$6 \angle 9 \varepsilon^{\circ} \mathrm{Et}$

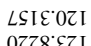

0ZZ8 EZ

L600 SCL

LOE6 'द

$\angle 9 Z S^{\circ} \angle Z L-T$
$\varepsilon 9 \angle S^{\circ} \angle Z I$

Z9ZLLZI

$9068^{\circ} \angle Z I$

$\angle \angle 06^{\circ} 82 \mathrm{I}$

69SZ'ZEI -

ZELE'EEI

9L6E"9عI

$\angle S 68^{\circ} 6 \mathrm{tI}$

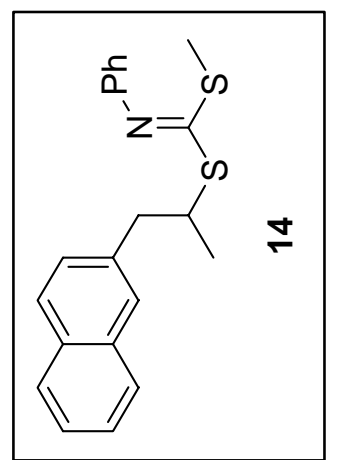




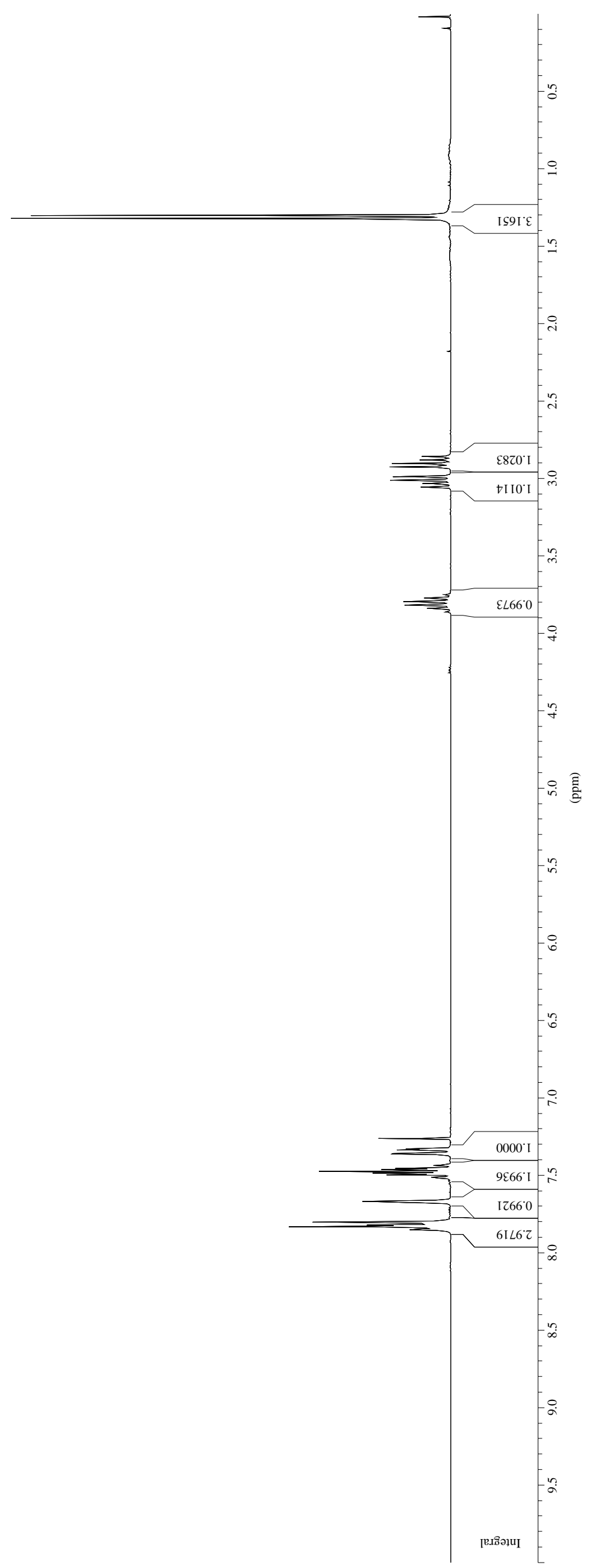


Z8EI'6I

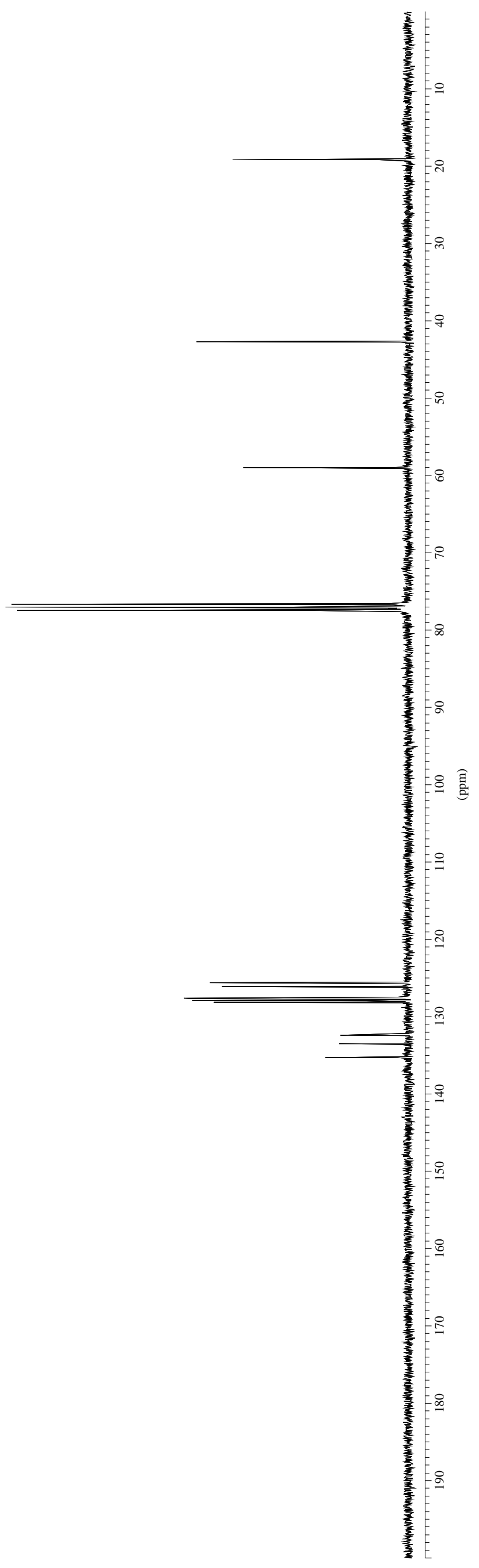

E8LS"9L

$0000 \mathrm{ZL}$

SI89: そt

เ6\&6.8S

言

9L6S'SZI

t0I'9ZI

SISC. $\angle Z T$

SZ†9'LZI

$0 t \angle 8 \circ Z \mathrm{I}$

9S0I.8ZI

8LtEZEL

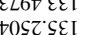

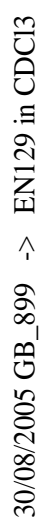

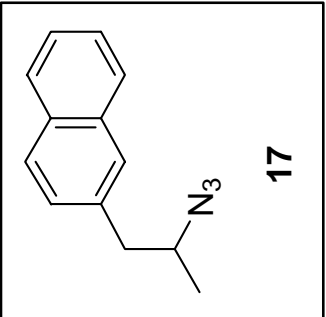




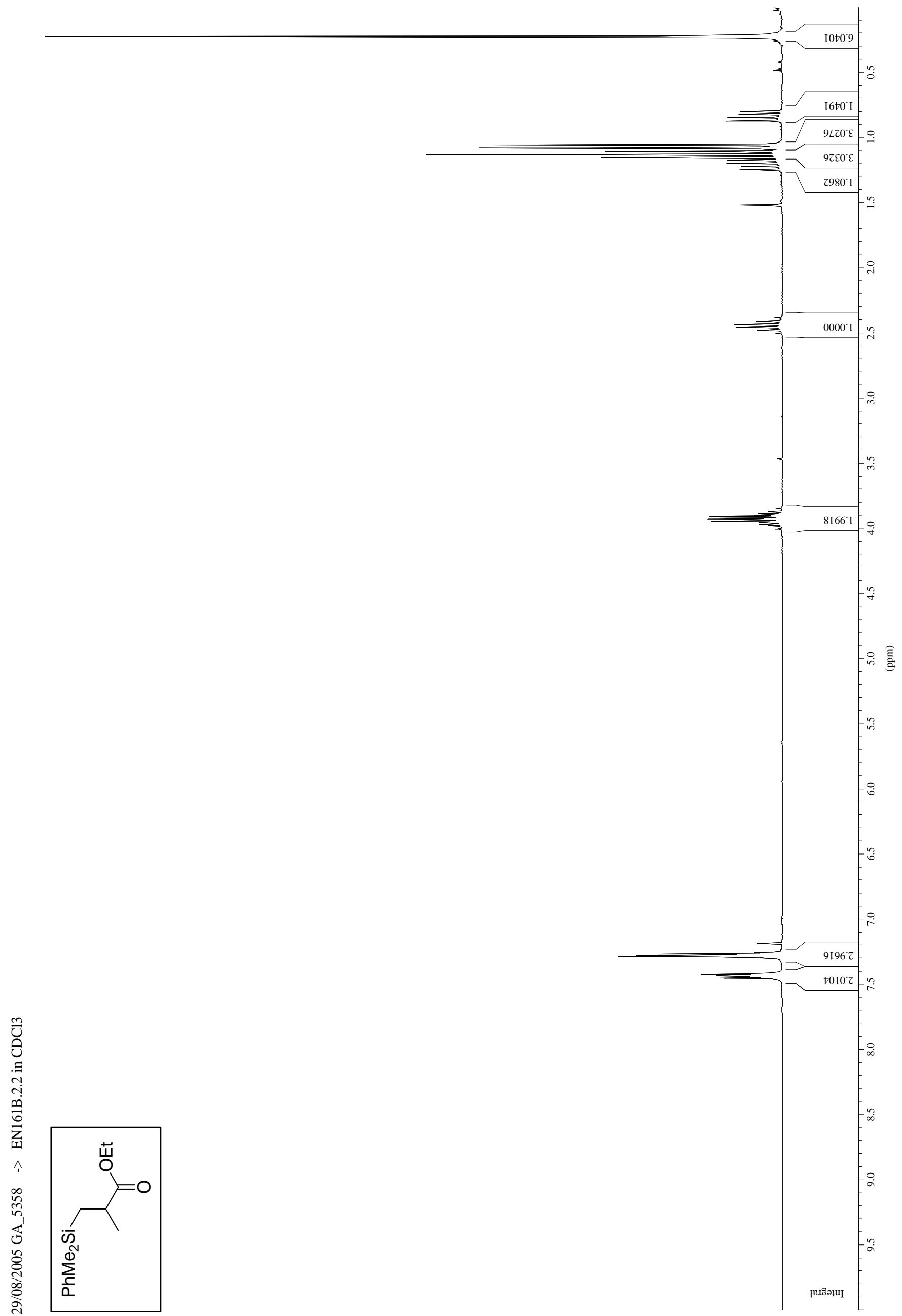


$986 t^{\circ} Z^{-}$

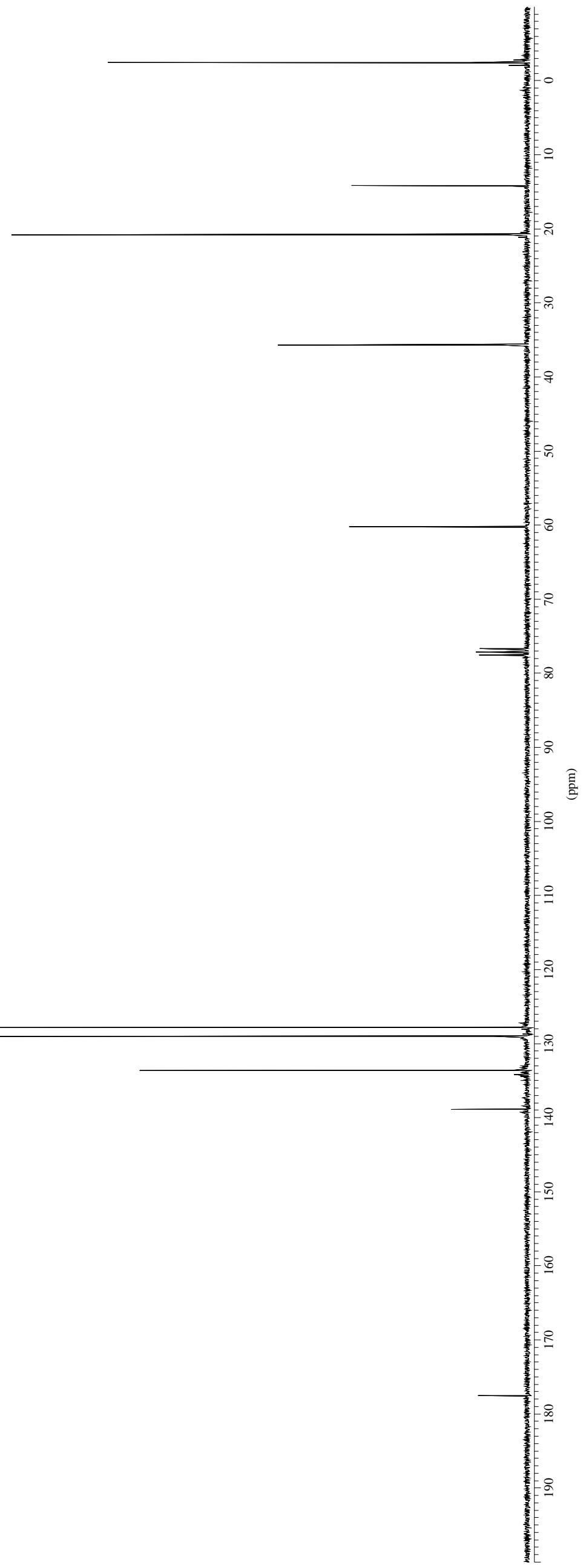

SStIำ

SZT $\angle \cdot 0 Z$

I69 $L^{\circ} 0 \mathrm{ZZ}$

ว8S9`ง

8โ99.9L -

$\angle Z 80^{\circ} L L$
$6 Z 05^{\circ} \angle L$

$8 Z 8 \angle ' \angle Z I$

L6L6'8ZI

†S9S EEI

I†T8.8EI

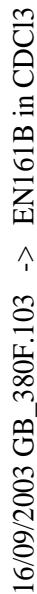

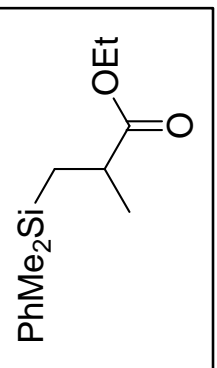




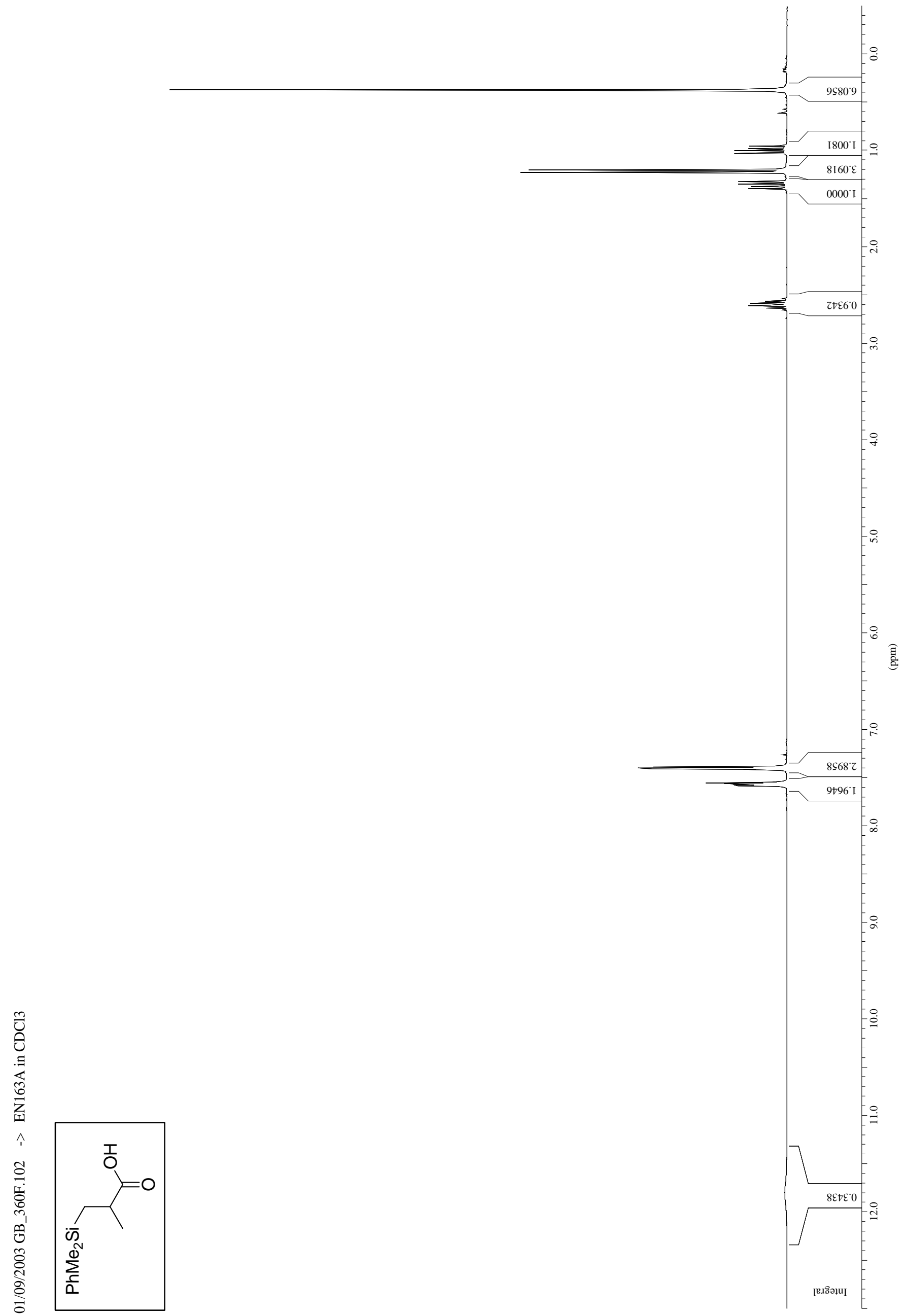


เ6z9' $z^{-}$

$\angle \angle 9 T^{\circ} Z^{-}$

9ZIL'0Z

SS9S $0 \mathrm{Z}$

ZIIS'SE

†TLS' $9 \angle$

$0000^{\circ} \angle L$

SL6L'LZI

$\angle 8100^{\circ} 6$ I I

E $\angle 0 S^{\circ} \varepsilon E I$

SIZS' 8 \&I

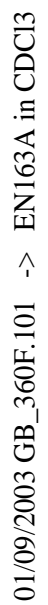

โ9Zガ๋8โ
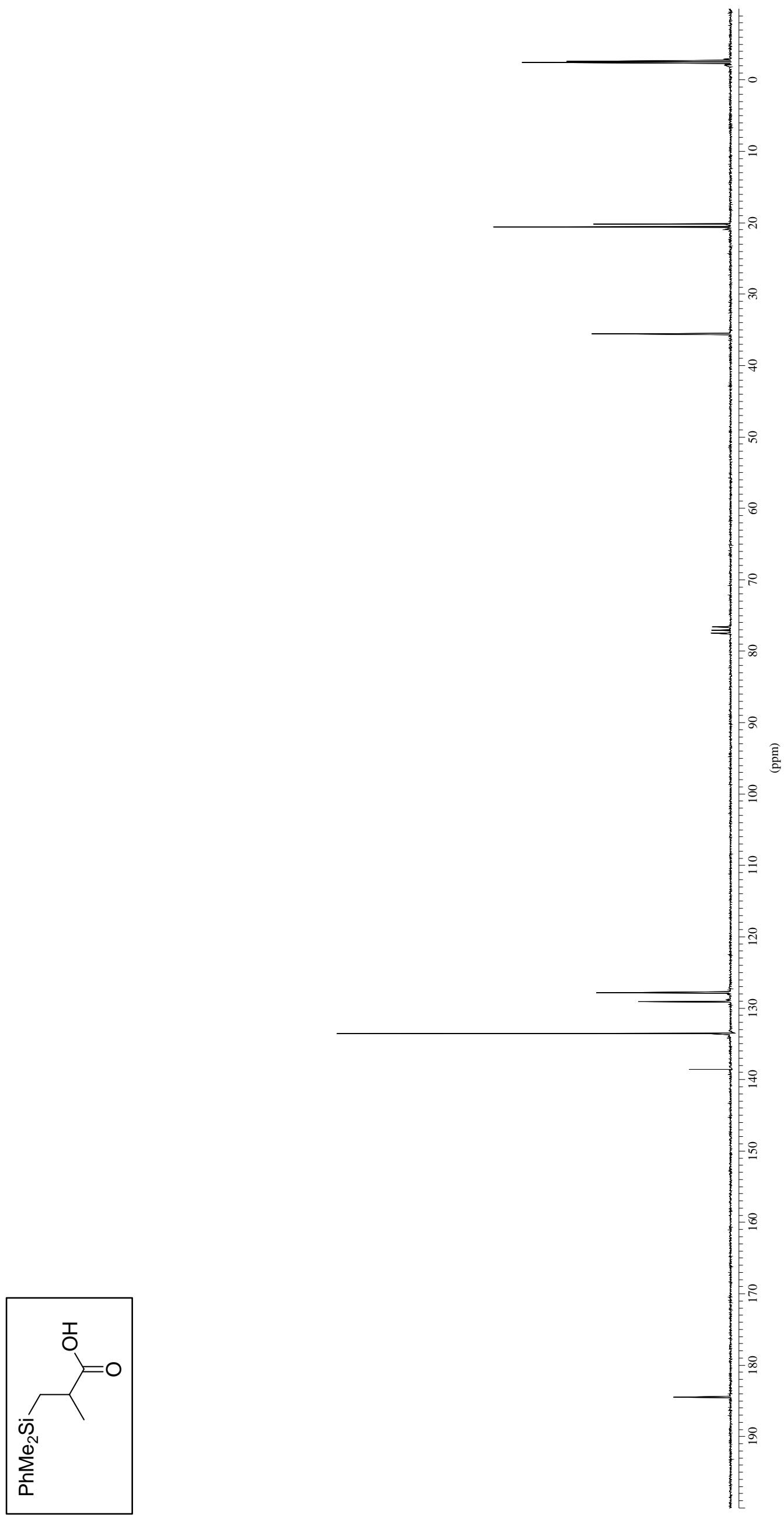


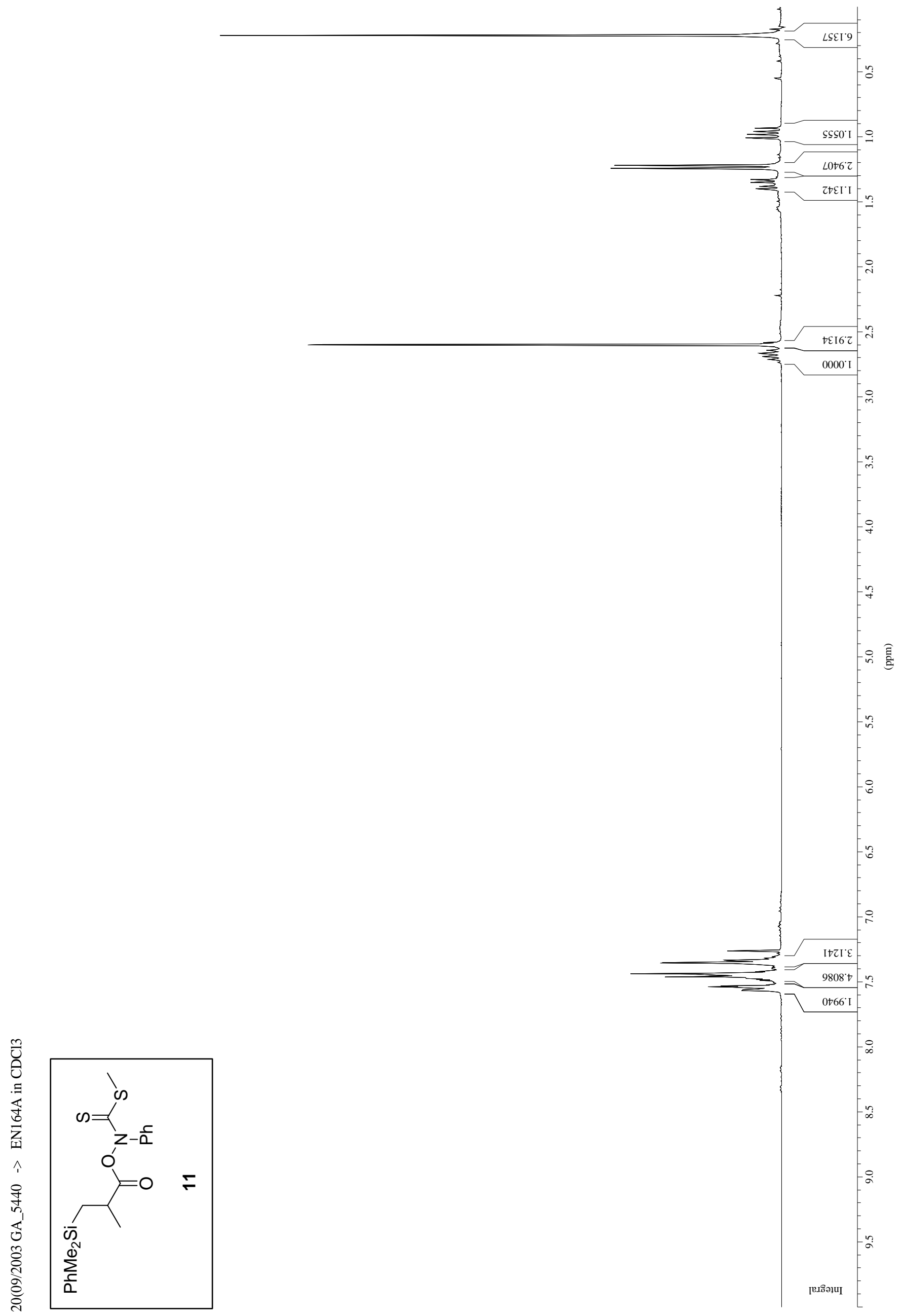




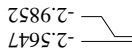

$90 t \angle \cdot 6 \mathrm{I}$

I8L8 $6 \mathrm{I}$

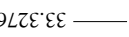

S6LS $9 \angle-$

$0000^{\circ} \angle L$

8SE9 $\angle Z I$

$\checkmark S \angle 6^{\circ} \angle Z I$

$\checkmark \angle 68^{\circ} 8 Z \mathrm{I}$

6๑E0.6ZI

6Z65'6ZI

9L9I'EEI

9L9I'EEI
EI9L'LEI

E966.0tI
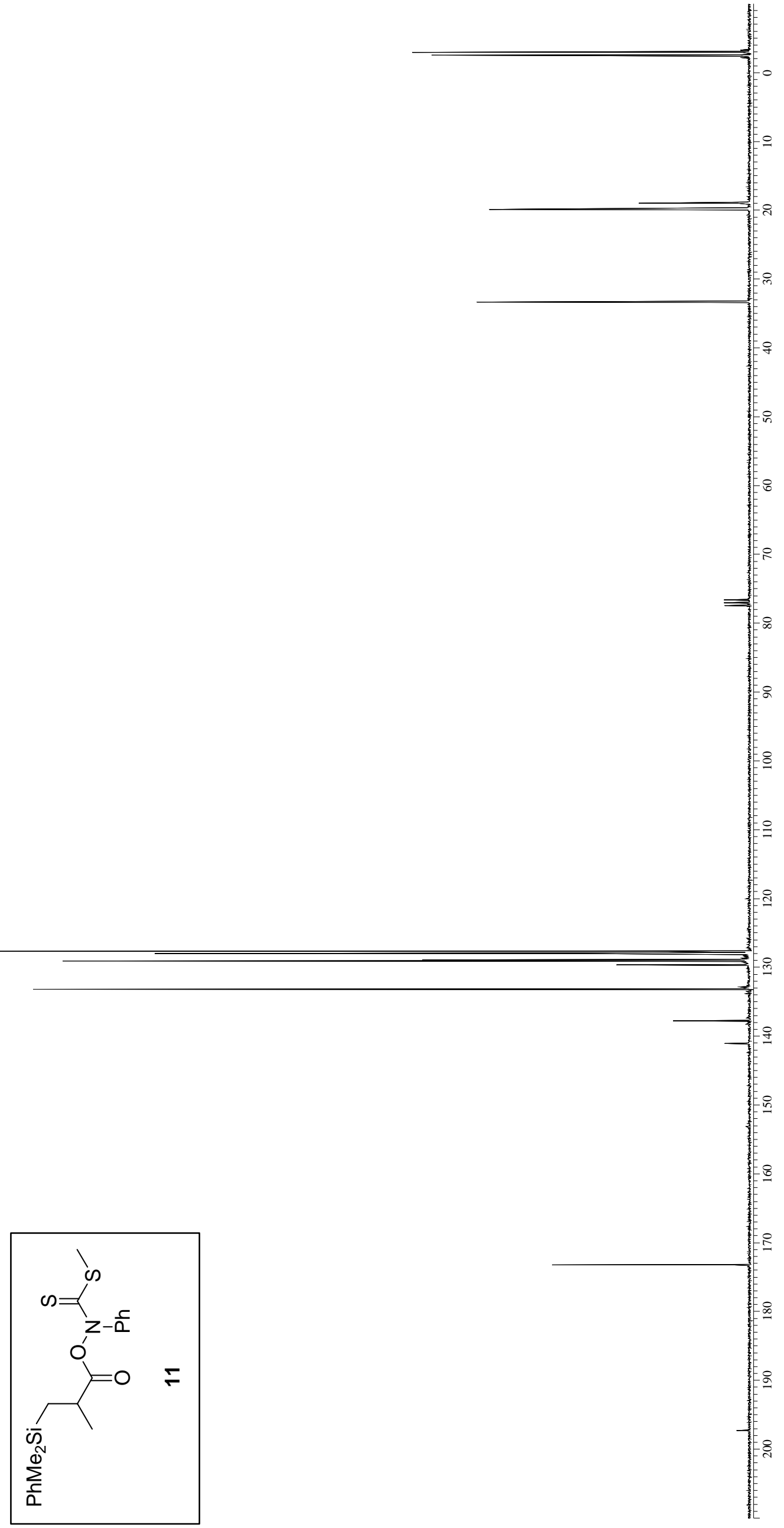

IZSI'ELI

$\angle 9 \& Z^{\circ} \angle 6 \mathrm{I}$

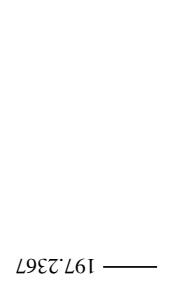

ֻ̊ 


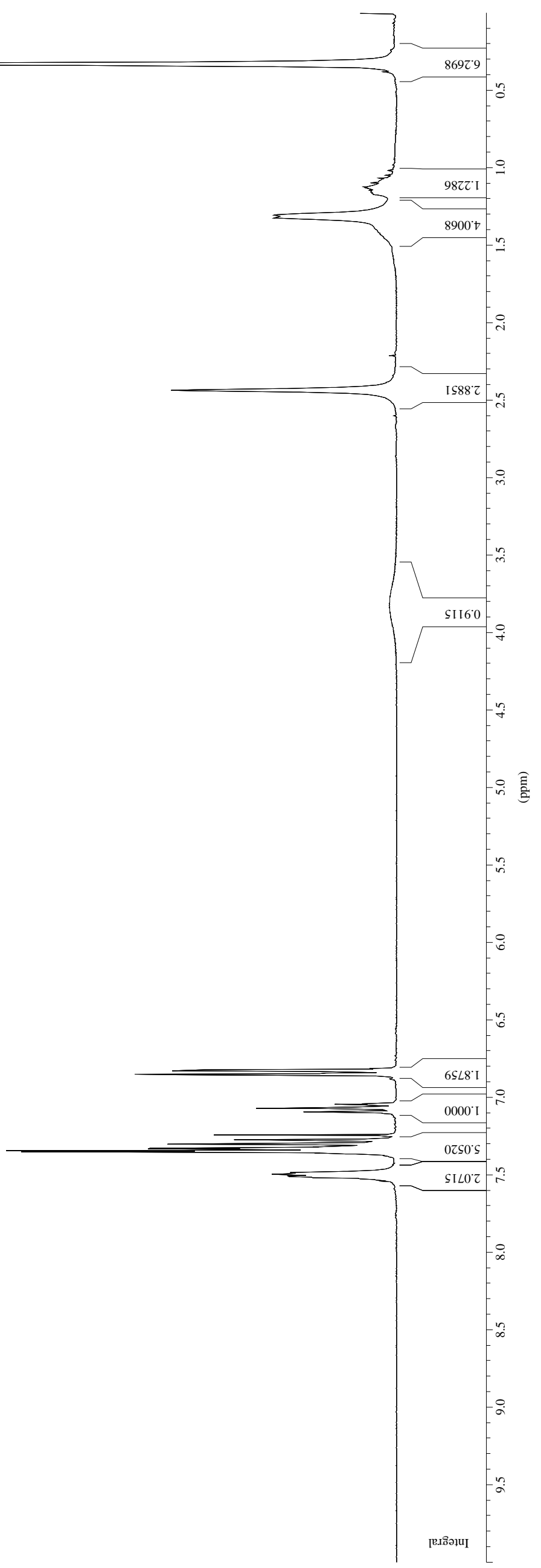


SILZ'Z-

SILZ2-

โ06z $\bullet \tau$

ยE8I'sZ -

Z\&99.6£

$00 \angle S^{\circ} 9 L$

$0000^{\circ} \angle L$

8IZナ゙LL

Z66Z'0ZI

6L69`¿ZI

$6 \angle 08^{\circ} \angle Z \mathrm{I}$

I8S8:8ZI

SEZ0'6ZI

IZZS ${ }^{\circ}$ E

60†9'8EI -

I8Z00ㄷ

60\&ร" เ9I
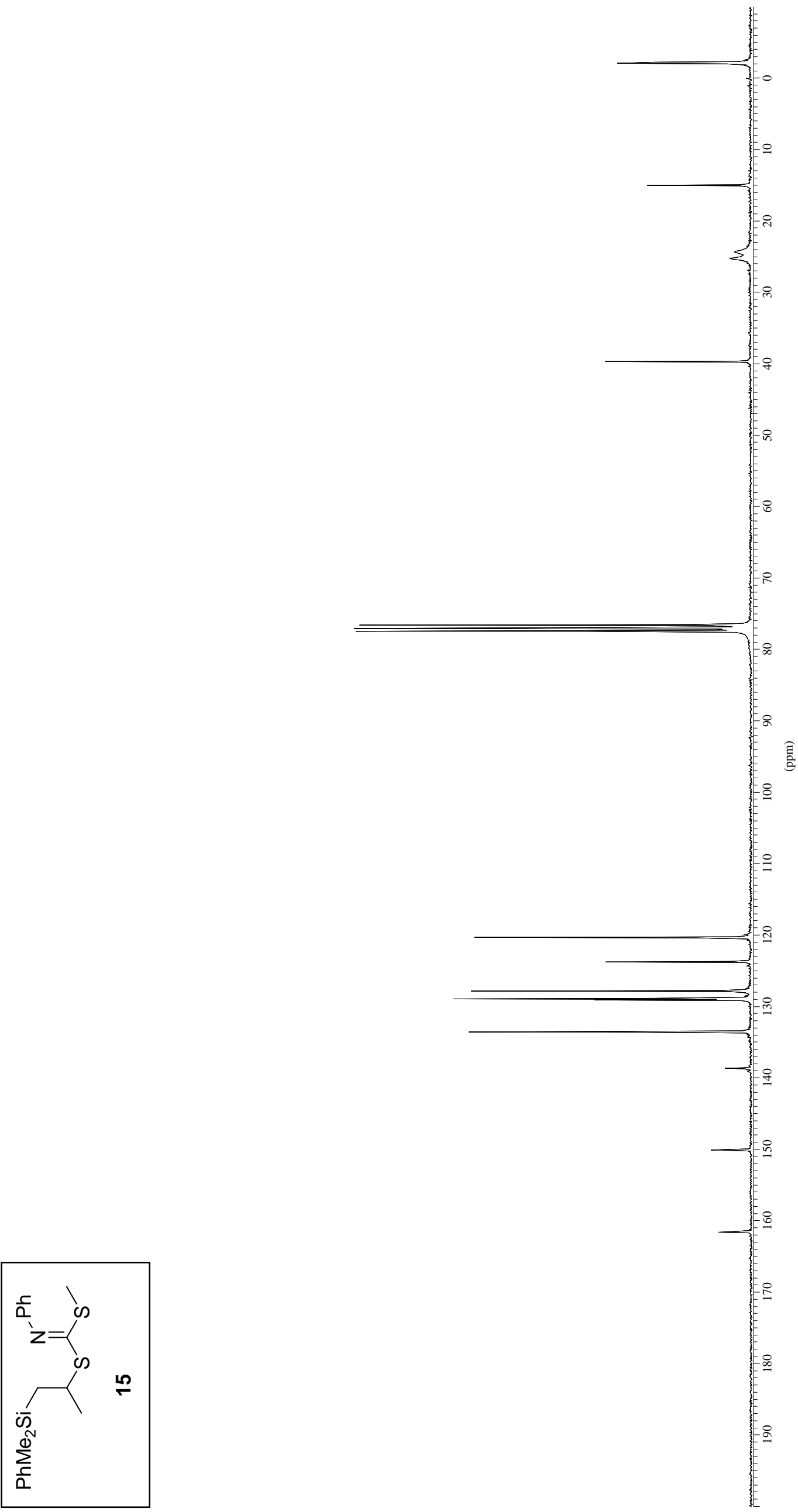


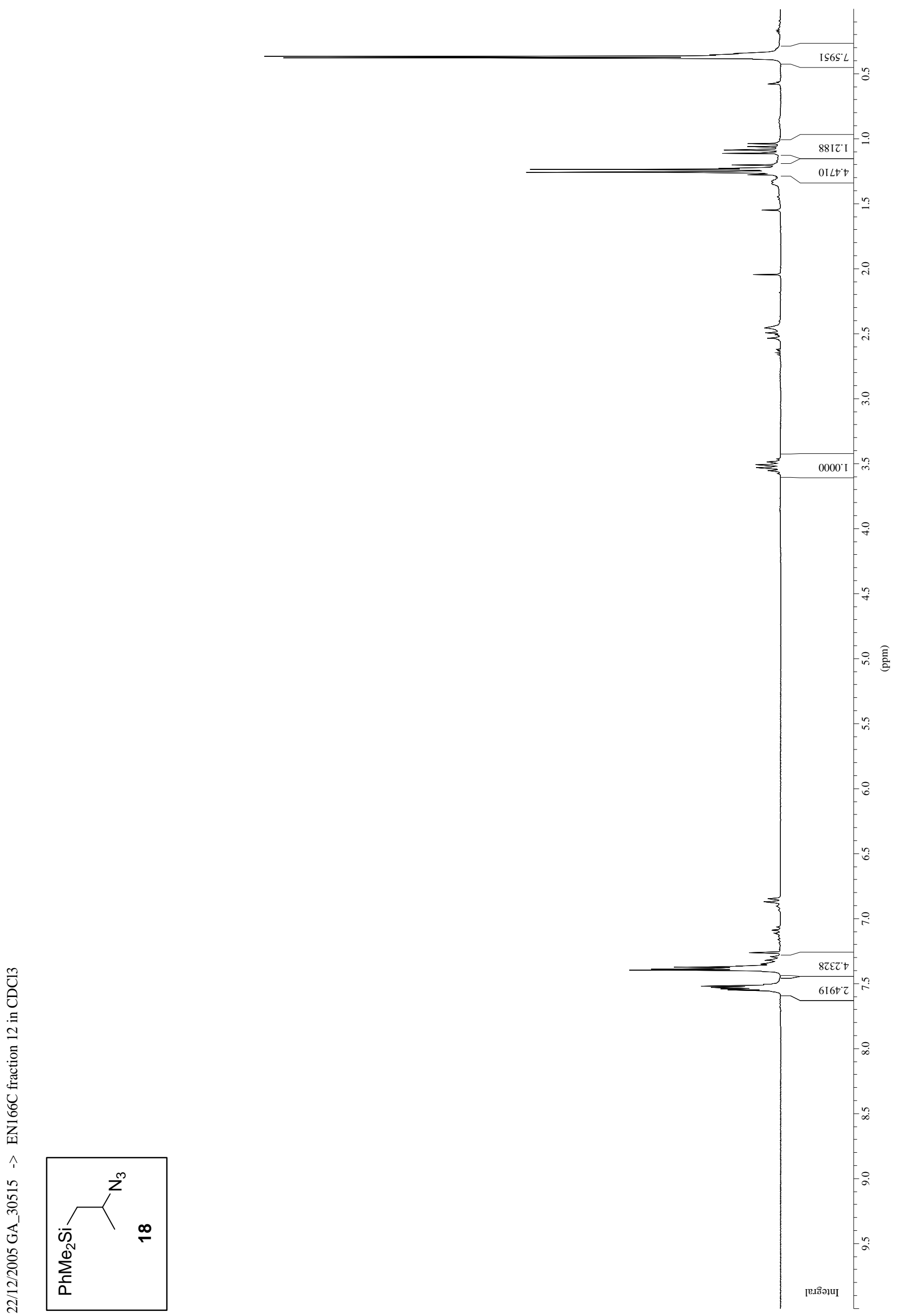




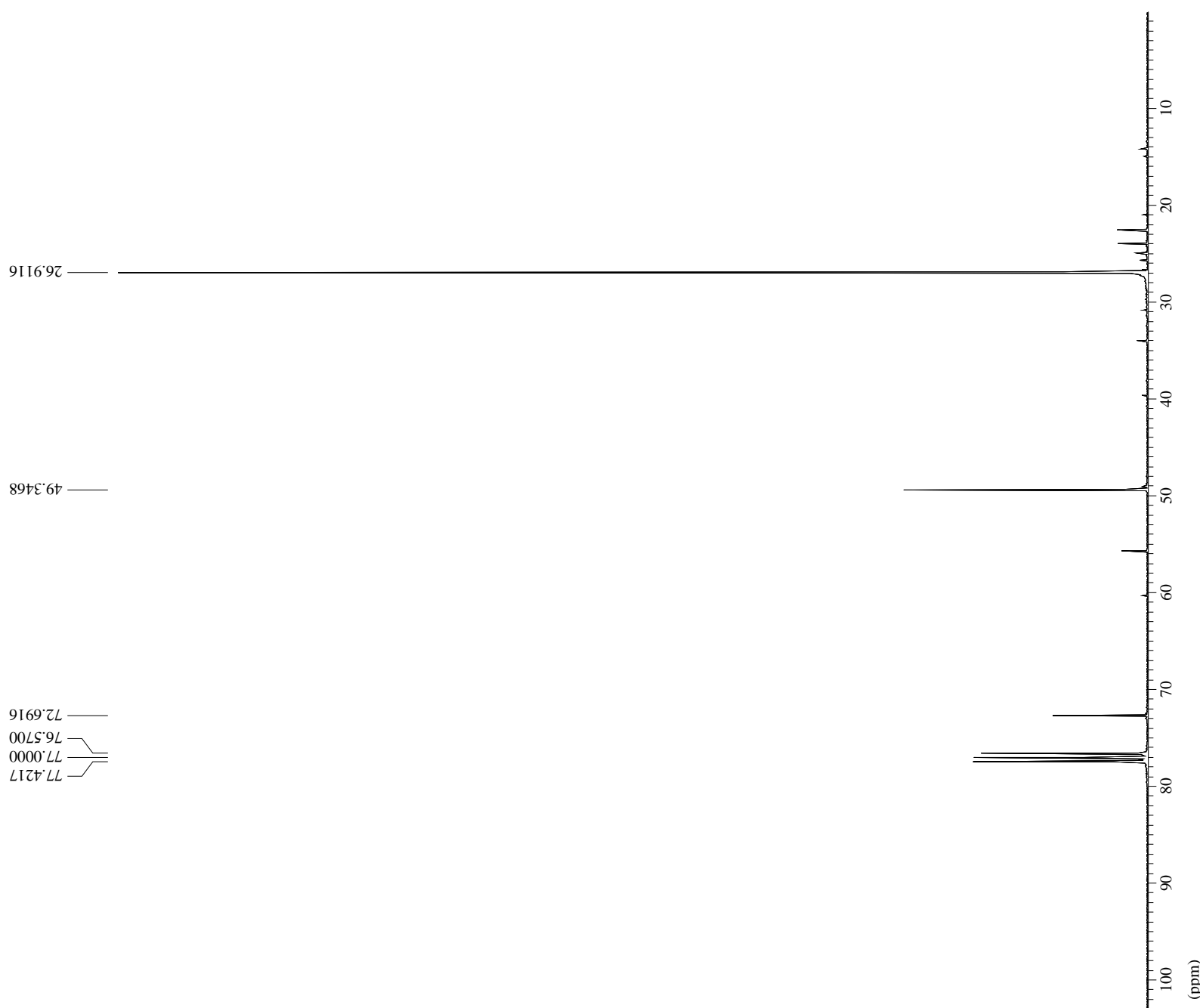

LLt'EEI

8LLI'8EI

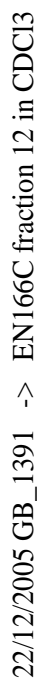

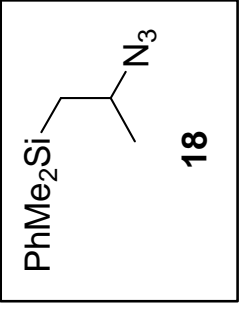




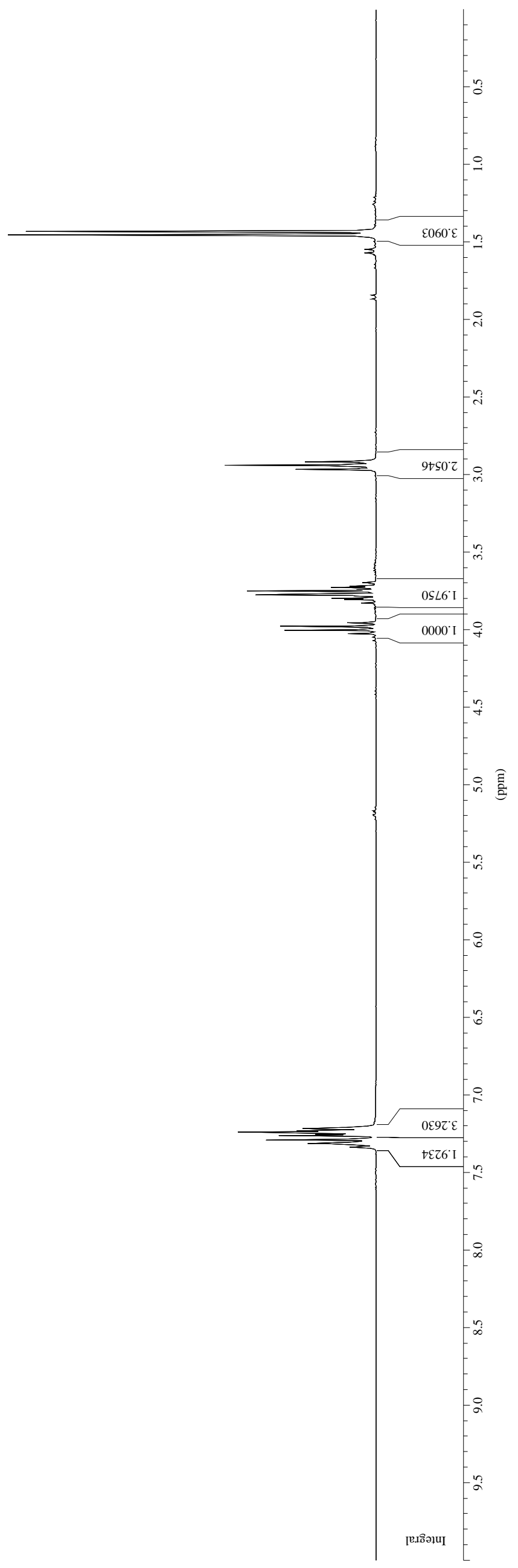


tSSI $8 \mathrm{I}$

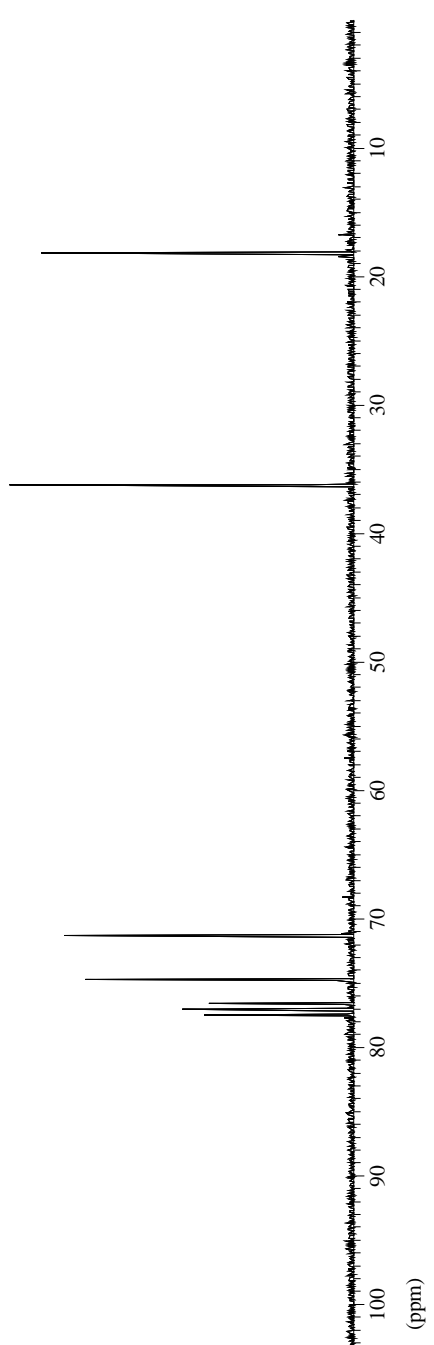

It $\angle Z^{\prime} I L$

$S 6 \angle S^{\circ} 9 L$
$0000^{\circ} \angle L$

$902 t^{\circ} L L$

LOEt 9 I L

9ZSt'8ZI

LZE8 8ZI

6โ8I'8EI

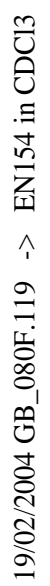

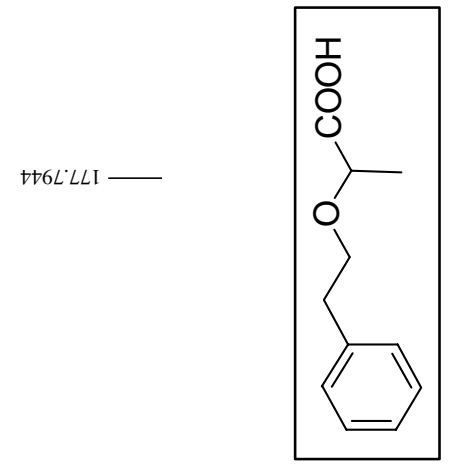

†十6L LLI 


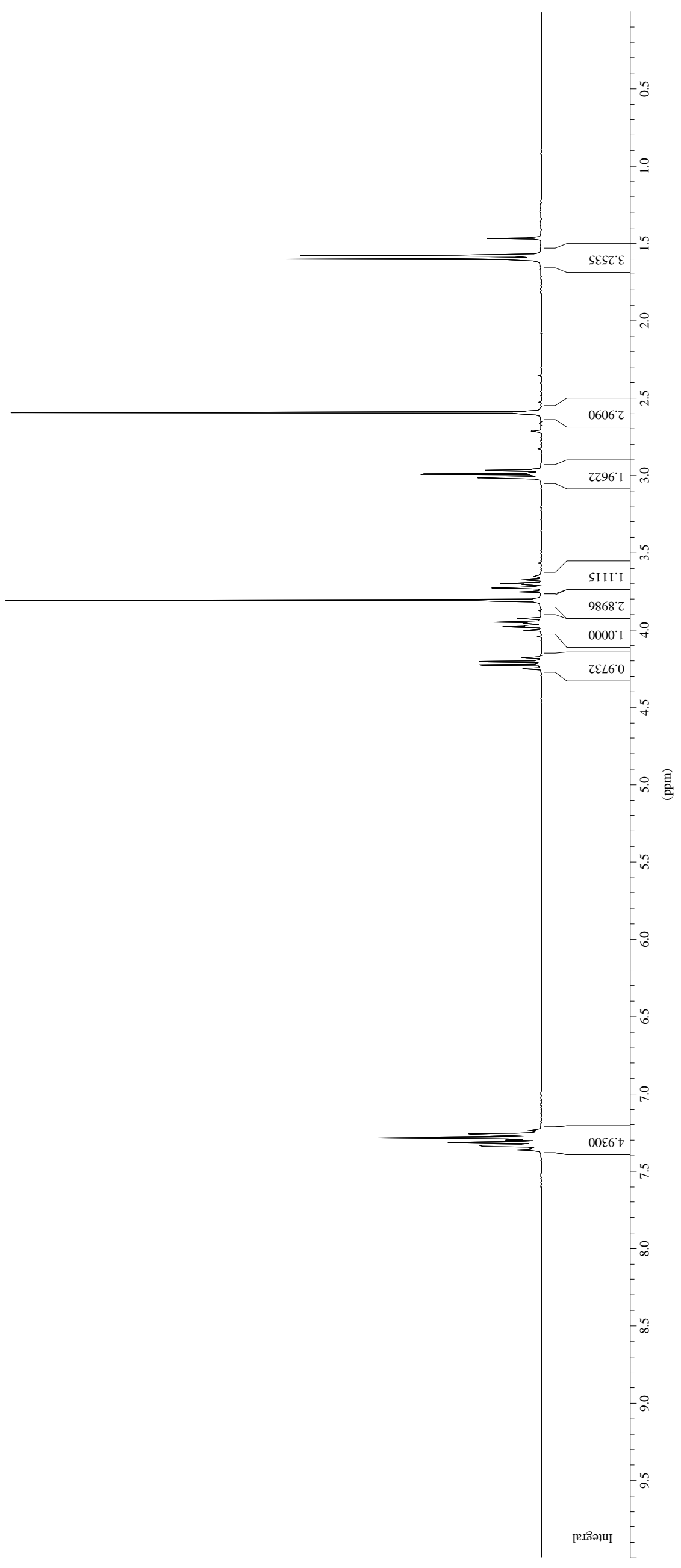


$\varepsilon 00 L^{\circ} \varepsilon L$

๑6 $\angle S^{\circ} 9 L$

9800

$66+\varepsilon * 92$

SL†E"8ZI

LS9I'8EI

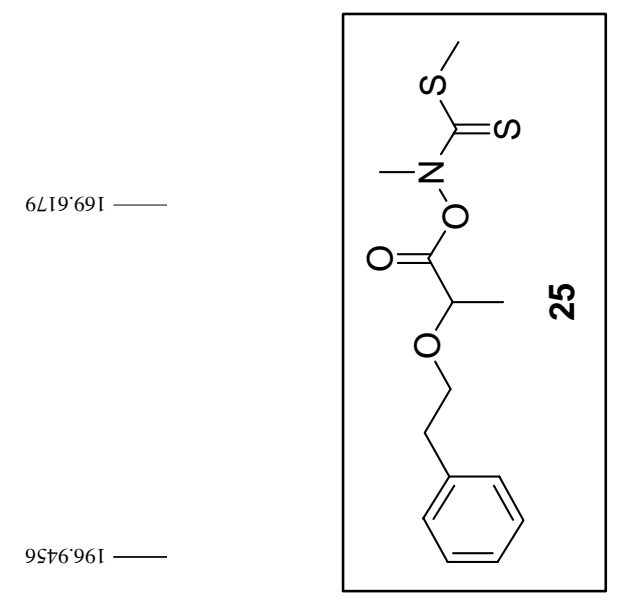



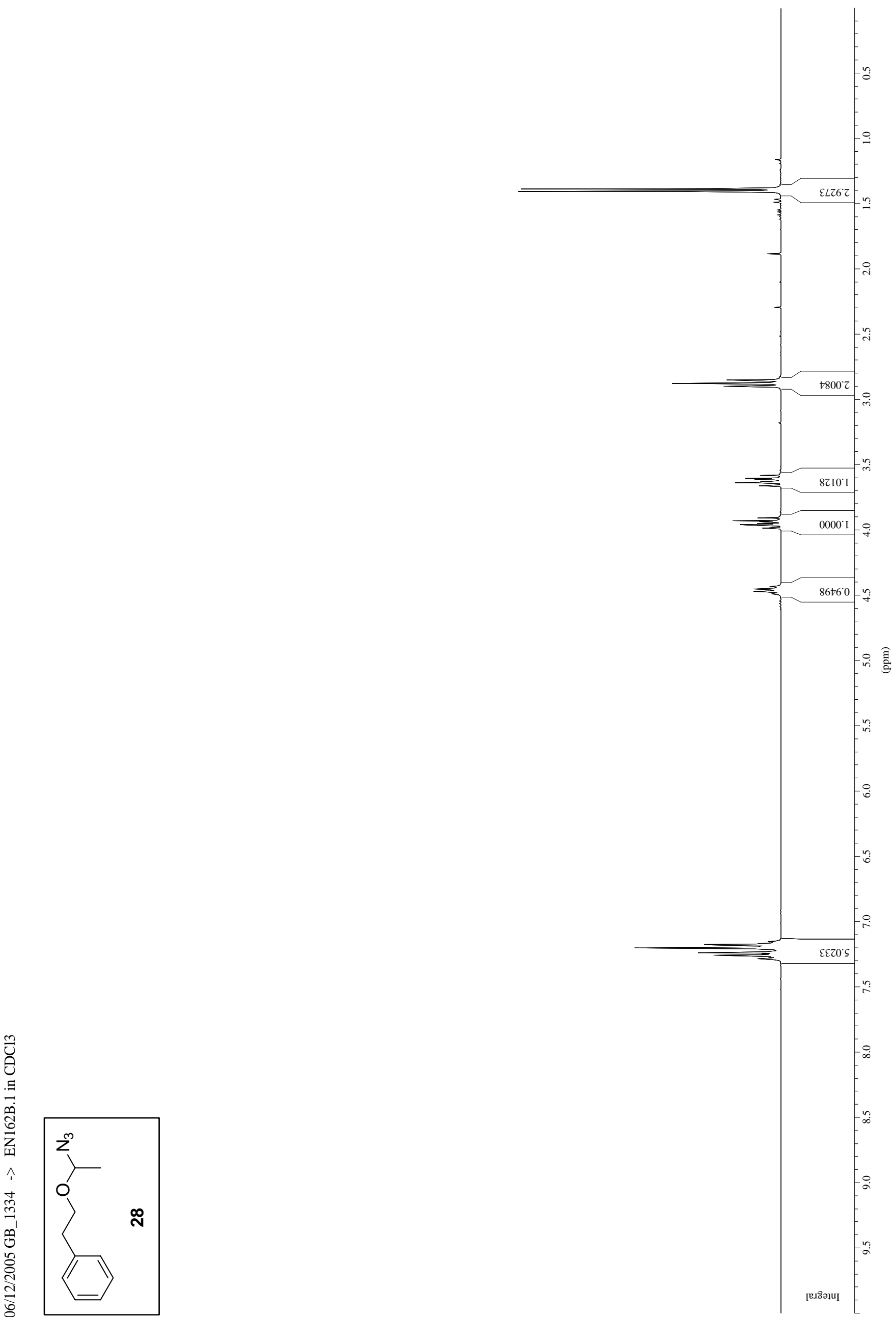
tSSI $0 Z$

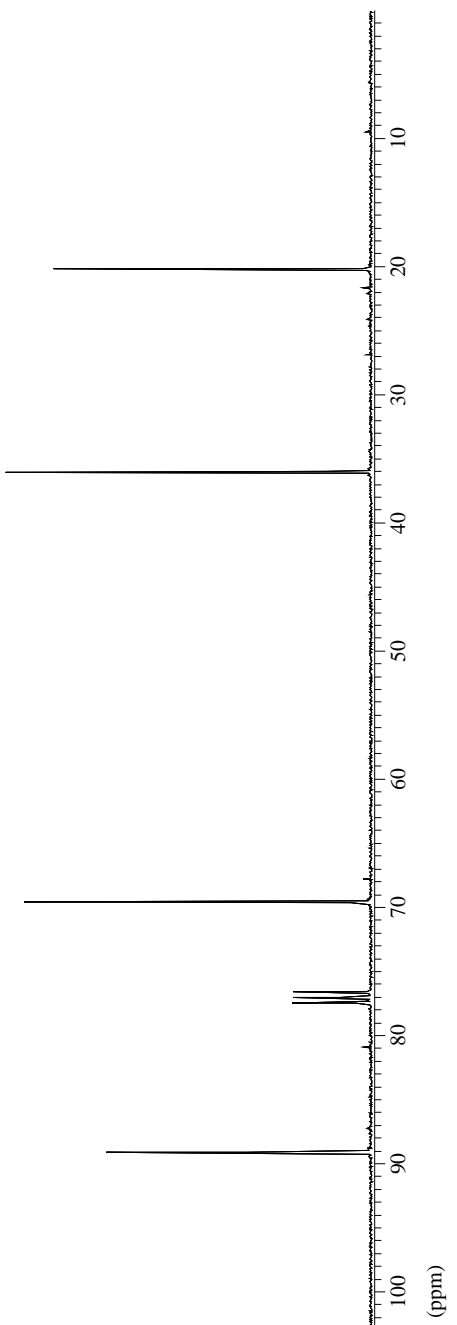

$\nabla \angle S S^{\circ} 69$

$00 \angle S^{\circ} 9 \angle$

$0000^{\circ} \angle L$

SI66 SE

†8เE'8ยโ

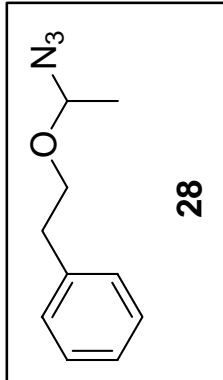




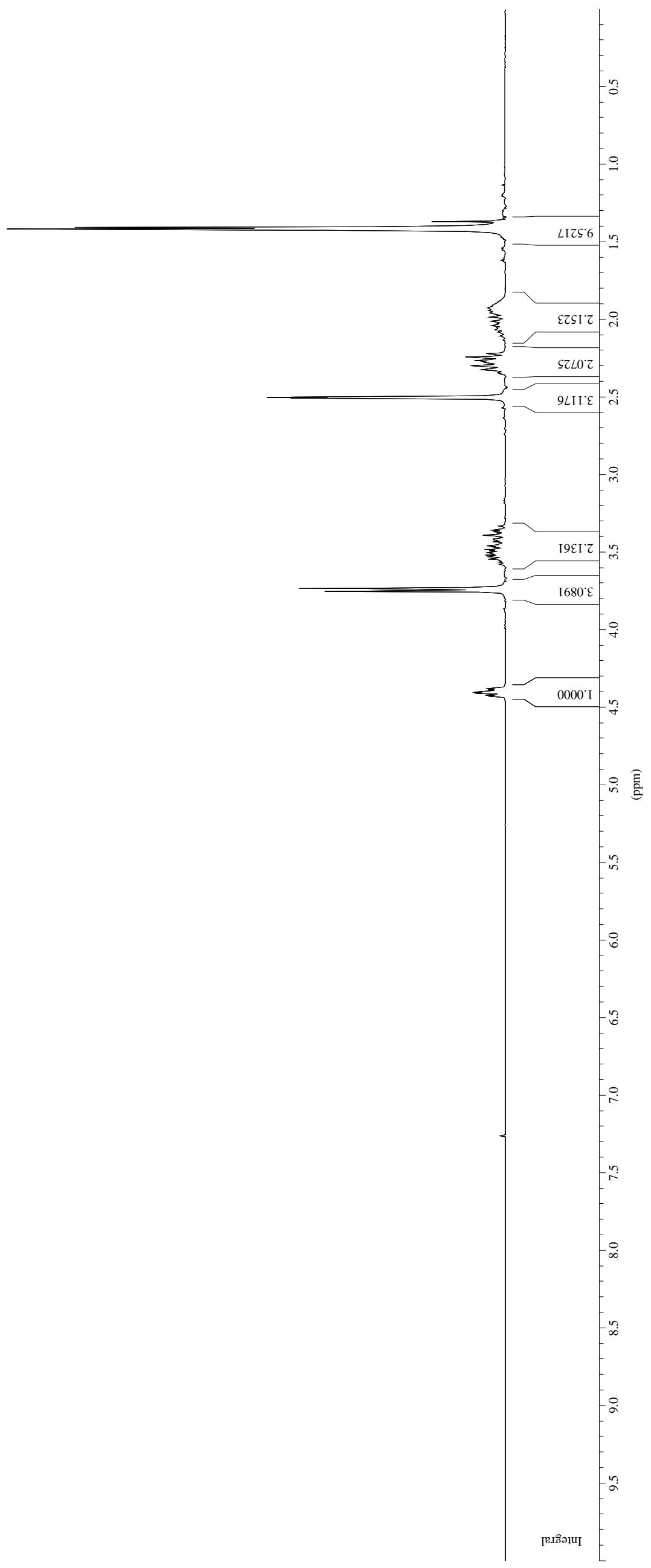


S8Et' $8 \mathrm{I}$

IS6t'8I

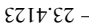

EOZS'

8SLI'8Z

$S 0 t Z^{\circ} 8 Z$
$\angle S \angle Z^{\circ} 6 Z$

ZZET 0 E

ててカだて

E8St $2 t$

EtS ${ }^{*} 9 t$
S9SE $9 t$

Z๐EZ' $\angle S$

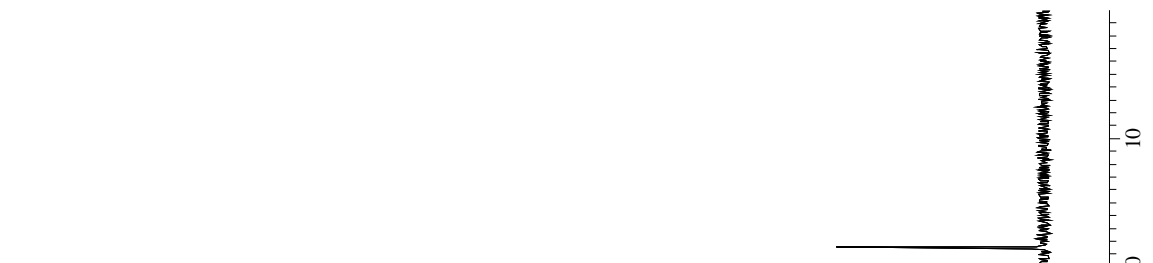

6LS" $9 L$

$0000^{\circ} \angle L$

SOZt $\angle L-$

ZISZ: 08

†โ9t:08

$\angle E D I^{\circ} E S I$

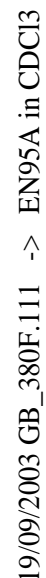

6650 69I

S9II'69I -

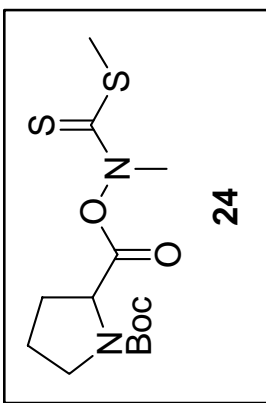




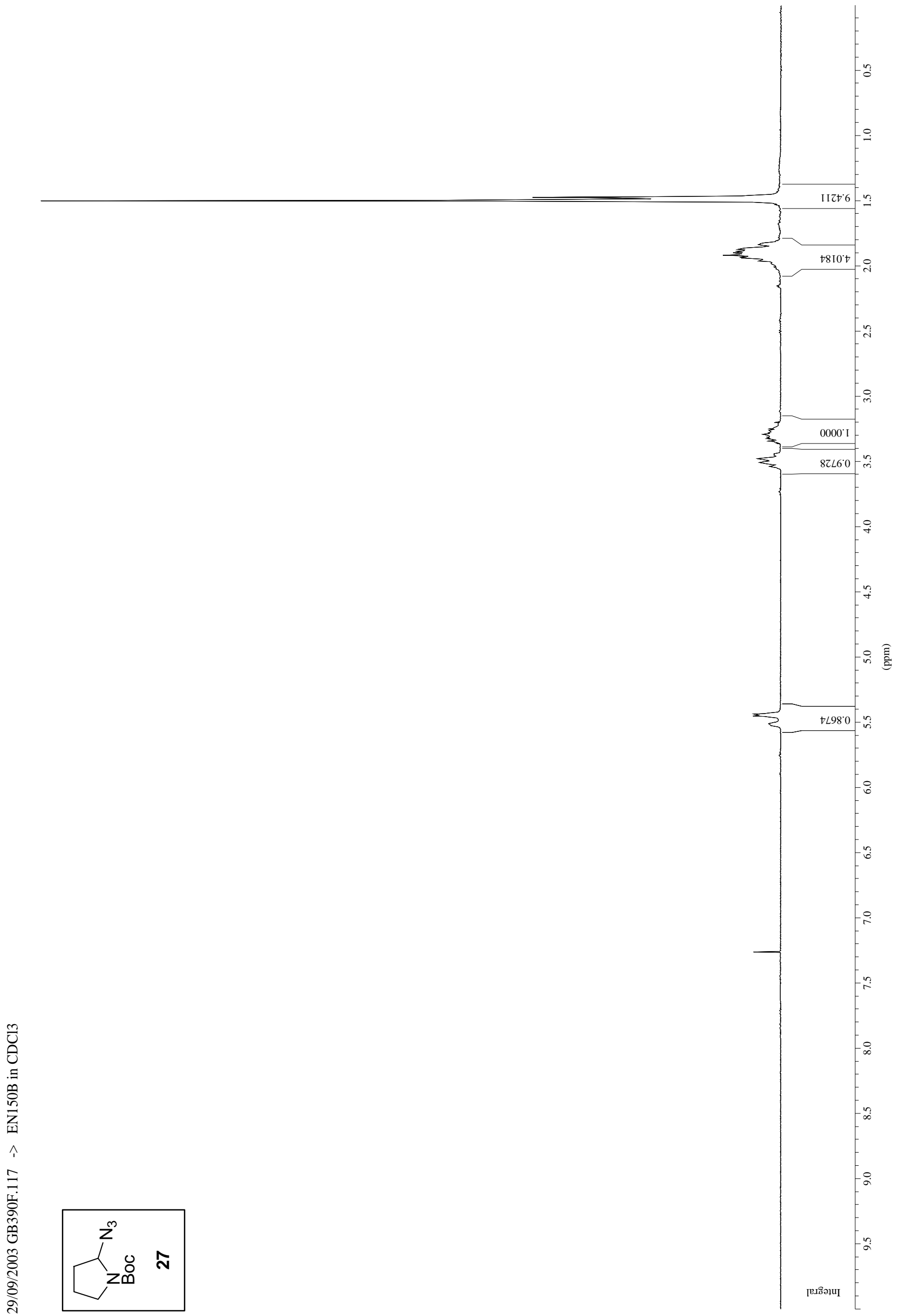


IS00 27

t656 22

†乙EZ: 82

ยเ8E'Zะ

เIE" $\varepsilon \varepsilon$

$\angle Z Z{ }^{\circ} \mathrm{S} t$

60I乙'9t

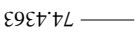

S6LS"9L

$0000^{\circ} L L-$

$98 Z \nabla^{\circ} \angle L$

0โZt 08

9๖ZI' 18
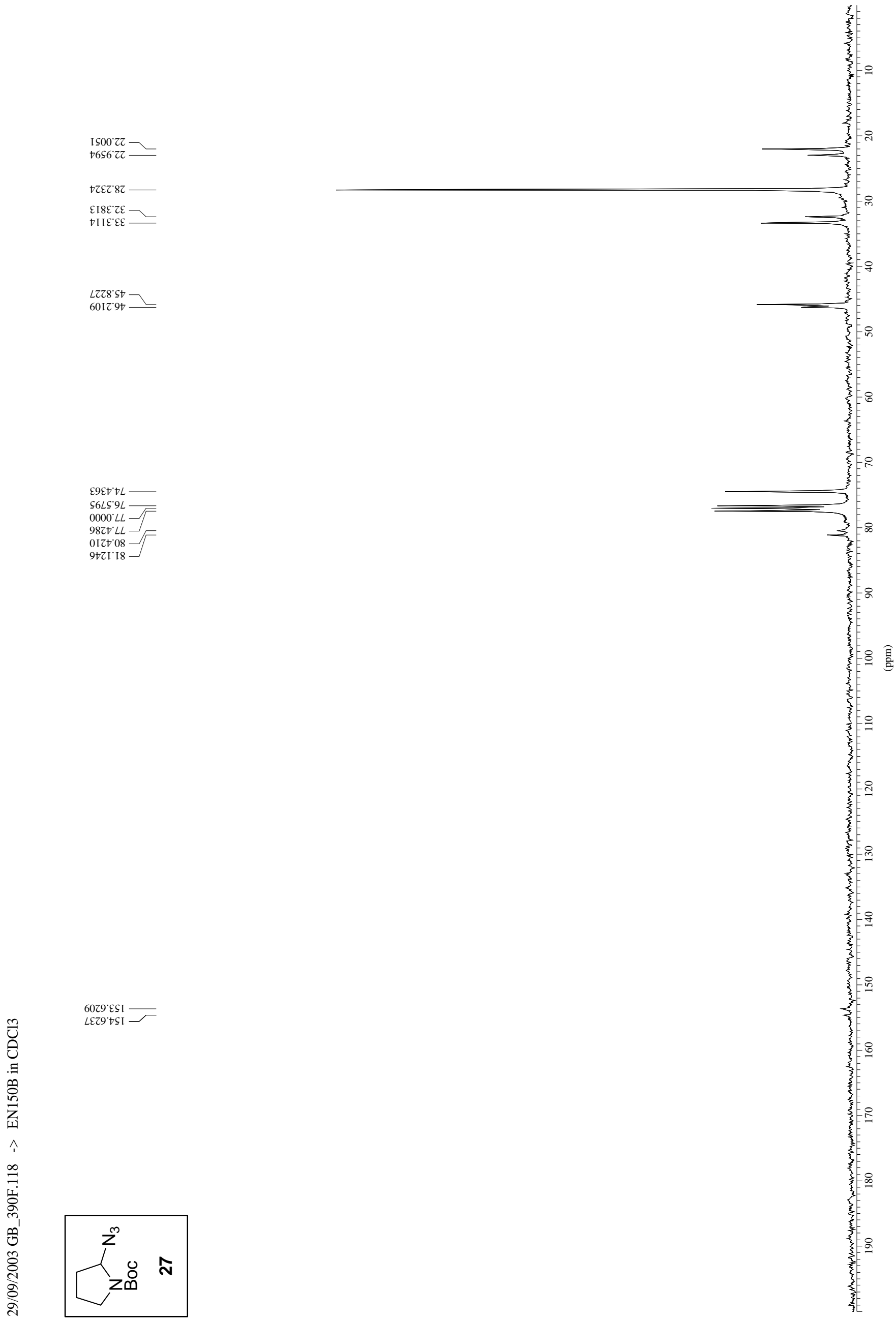


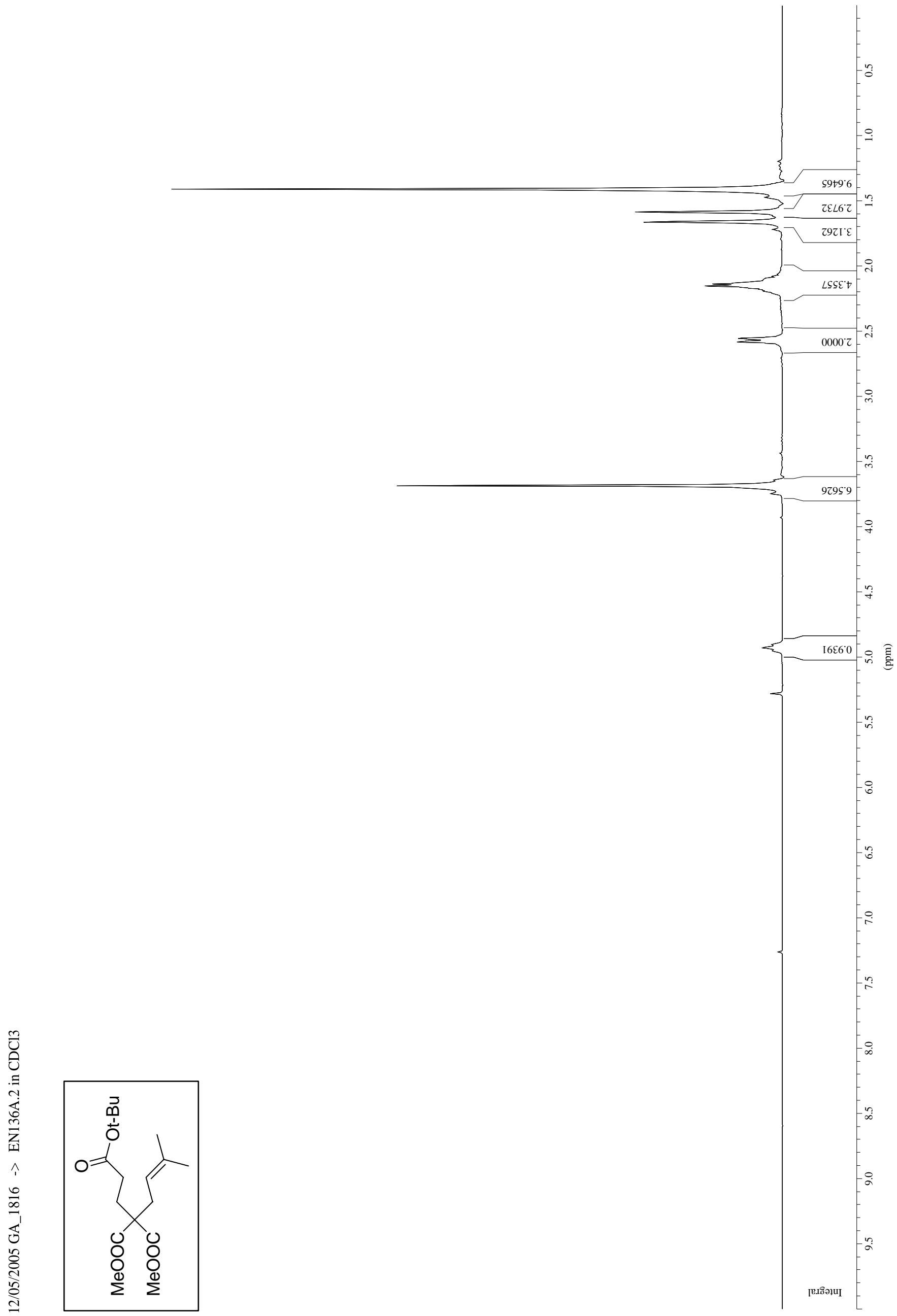


$966 L^{\circ} \angle I$

8IS6 ${ }^{\circ} \mathrm{SZ}$

Z8S9 $\angle Z$

$0900^{\circ} 8 Z$
$6 Z 89^{\circ} 0 \varepsilon$

66 ${ }^{\circ}$ 'IE

乙E\&E'ZS

9I66.9S

S6LS'9L

$0000^{\circ} \angle L$

98Zt'LL

DISZ $\angle I I$

ZI06'SEI —

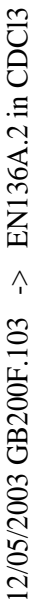
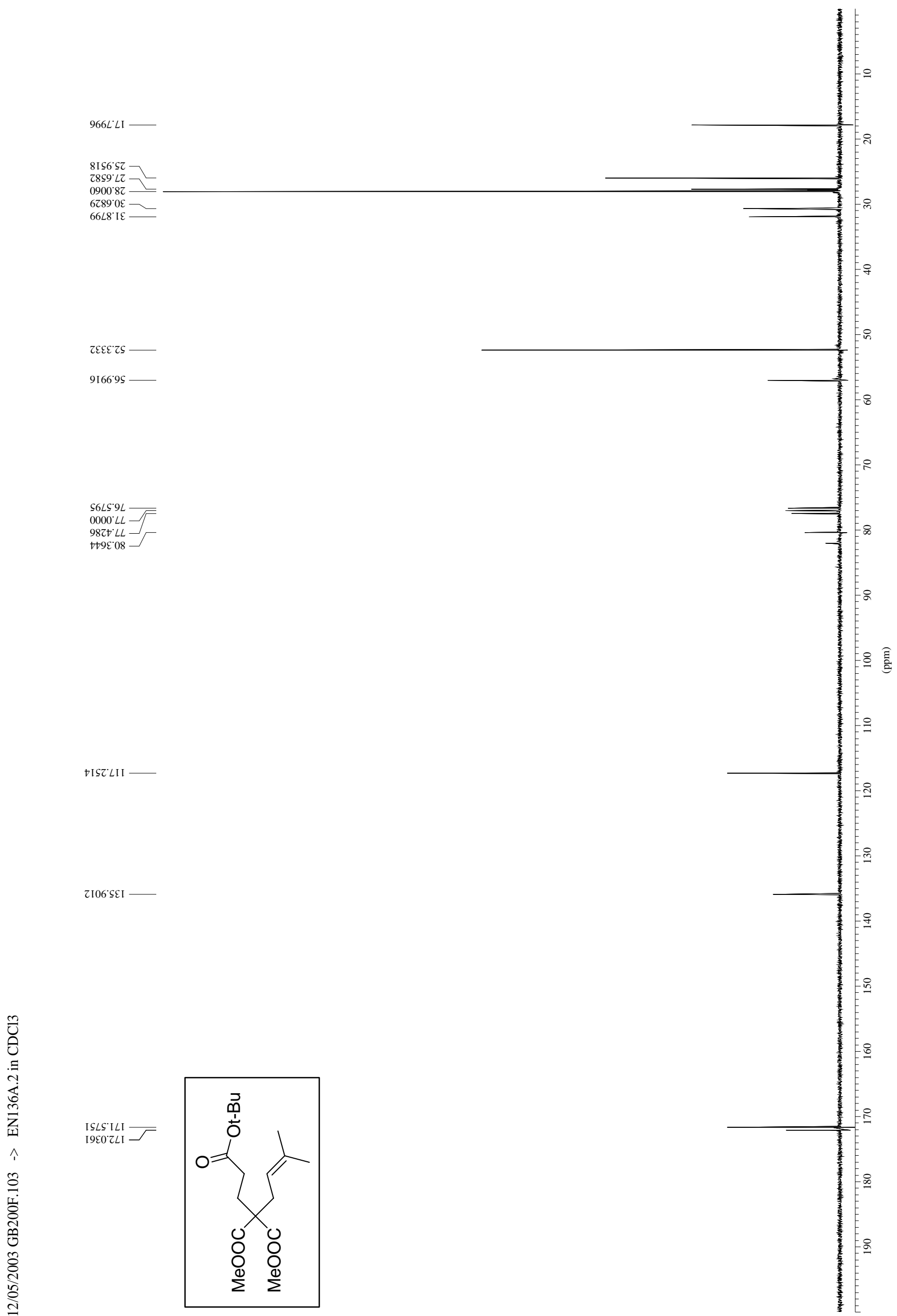


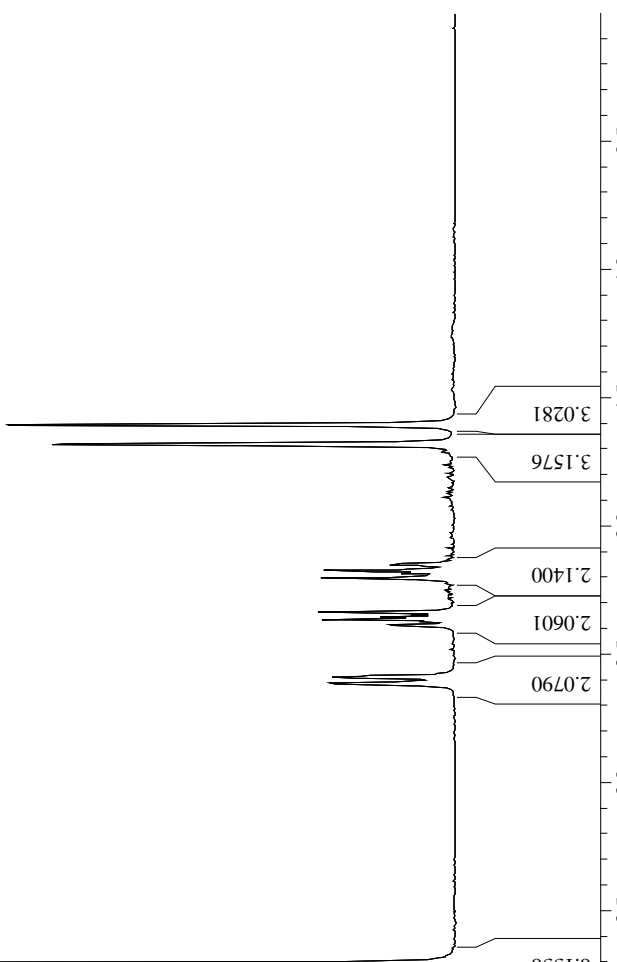


$\downarrow \varepsilon \& L^{\circ} \angle I$

ELI6"SZ

t\&9L'LZ

†0เย'6乙

IE66 ${ }^{\circ}$ IE

868E'ZS

SL6L'9S

†I $\angle S^{\circ} 9 \angle$

$0000^{\circ} L L$

9Z66"9II

6ISI'9EI

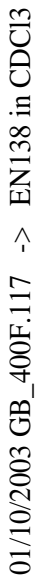
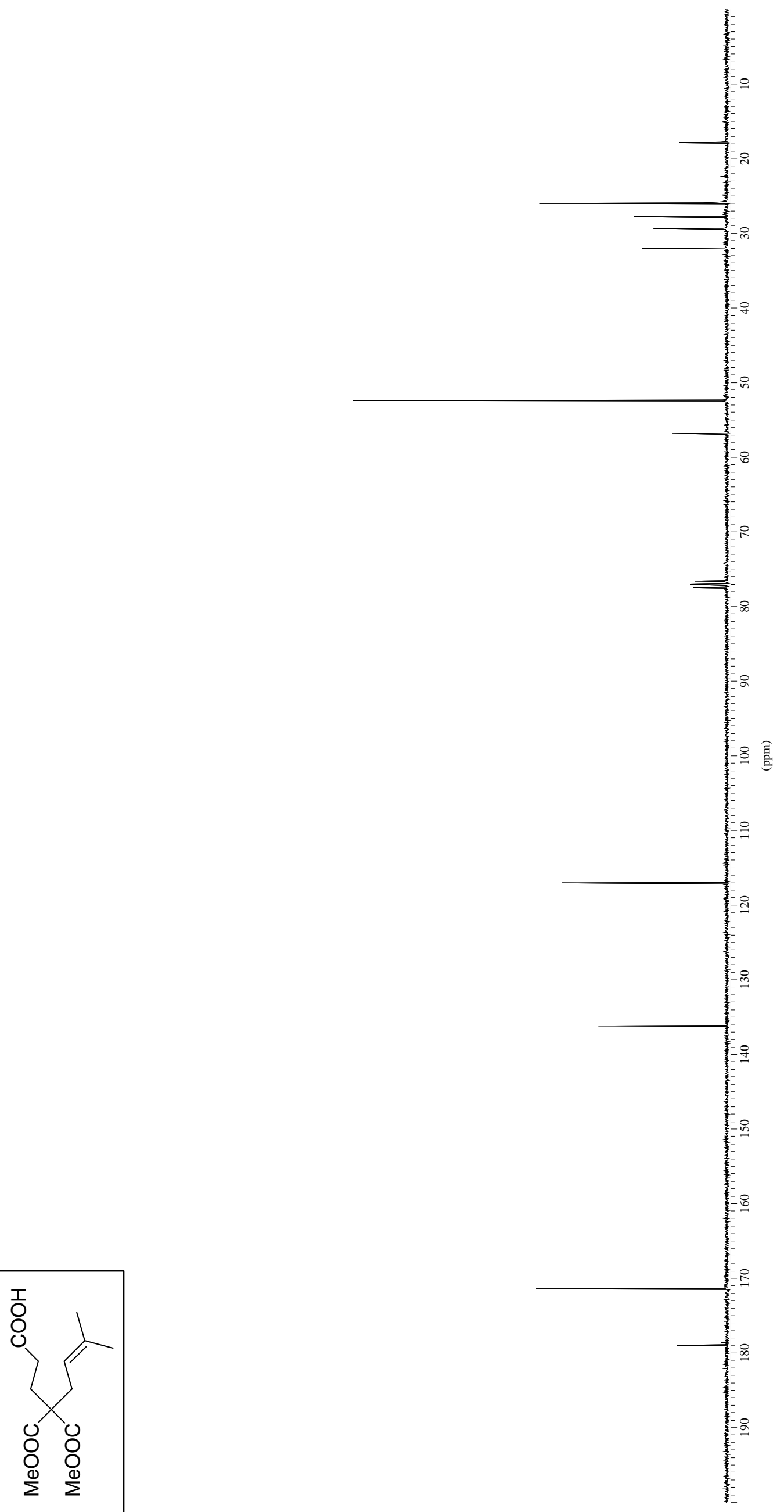


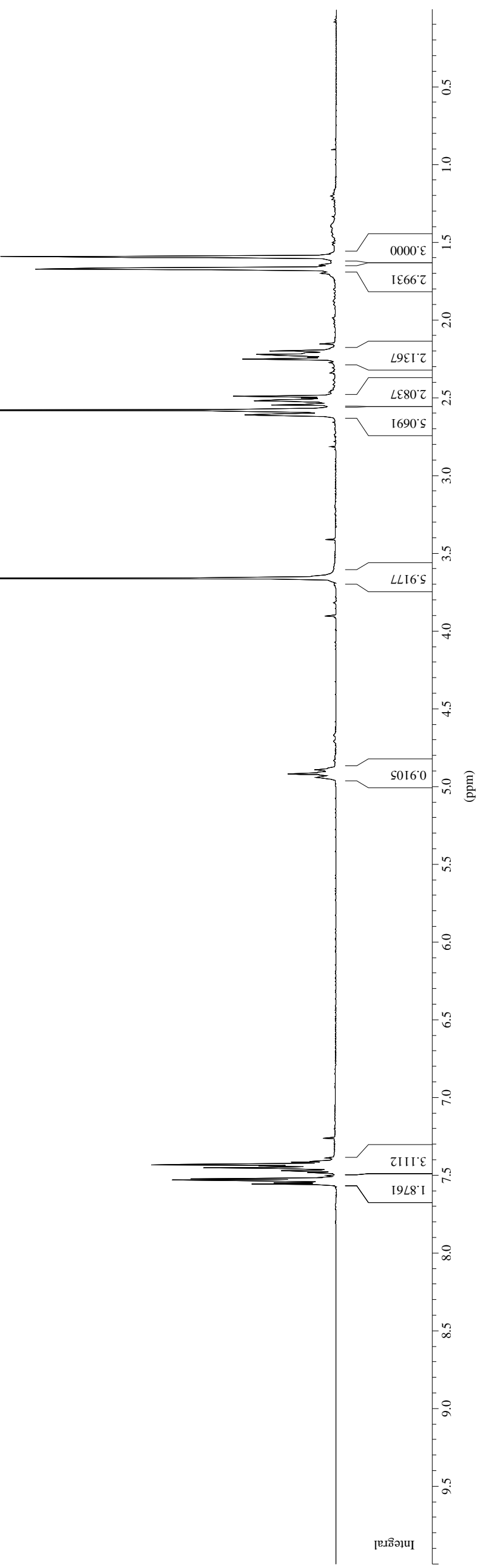


$\angle L Z Z ' Z \varepsilon$

6L6E'ZS

†00L'9S

tILS" $9 L$

$0000^{\circ} \angle L$

90Zt'LL

8ZZ8'9II
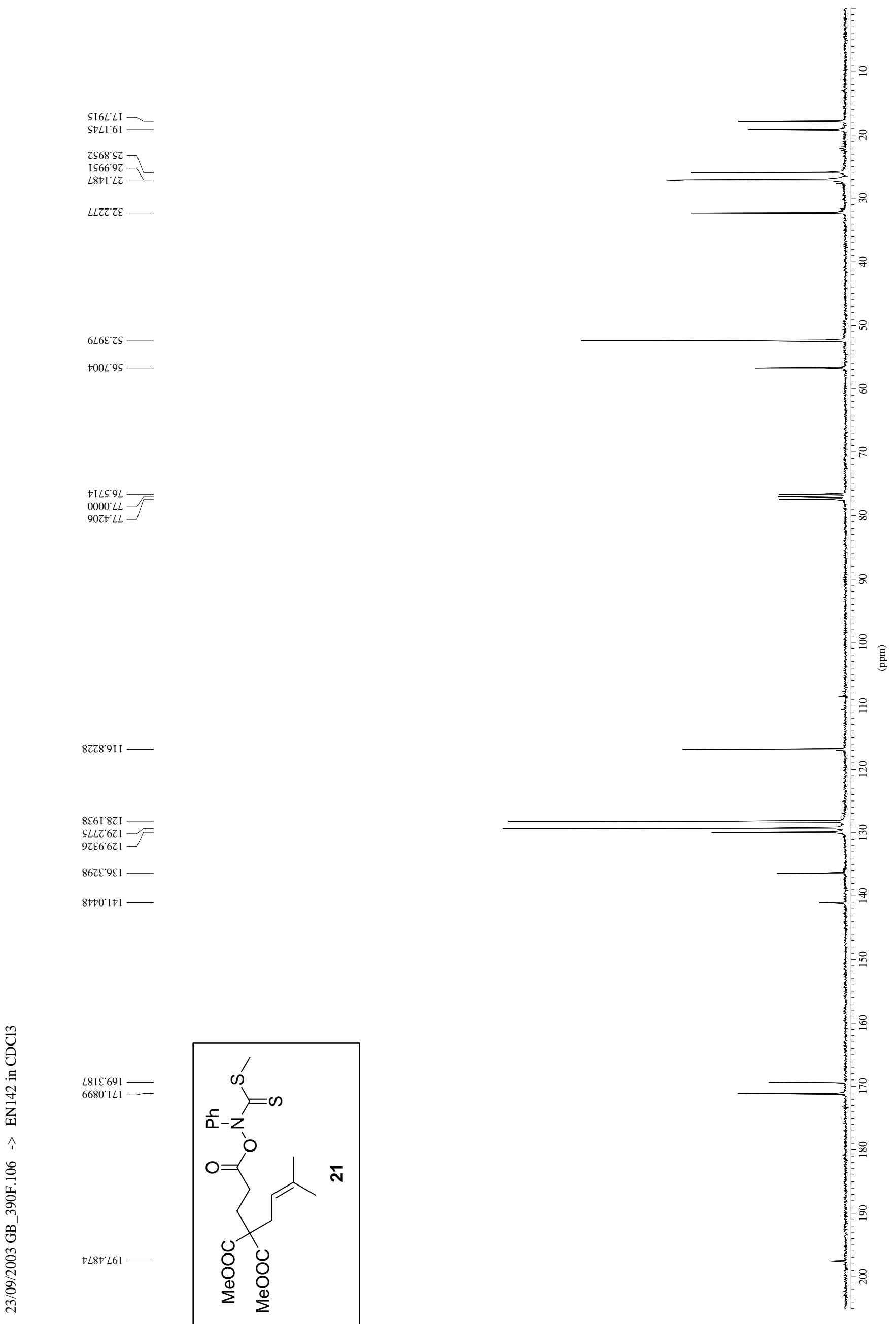


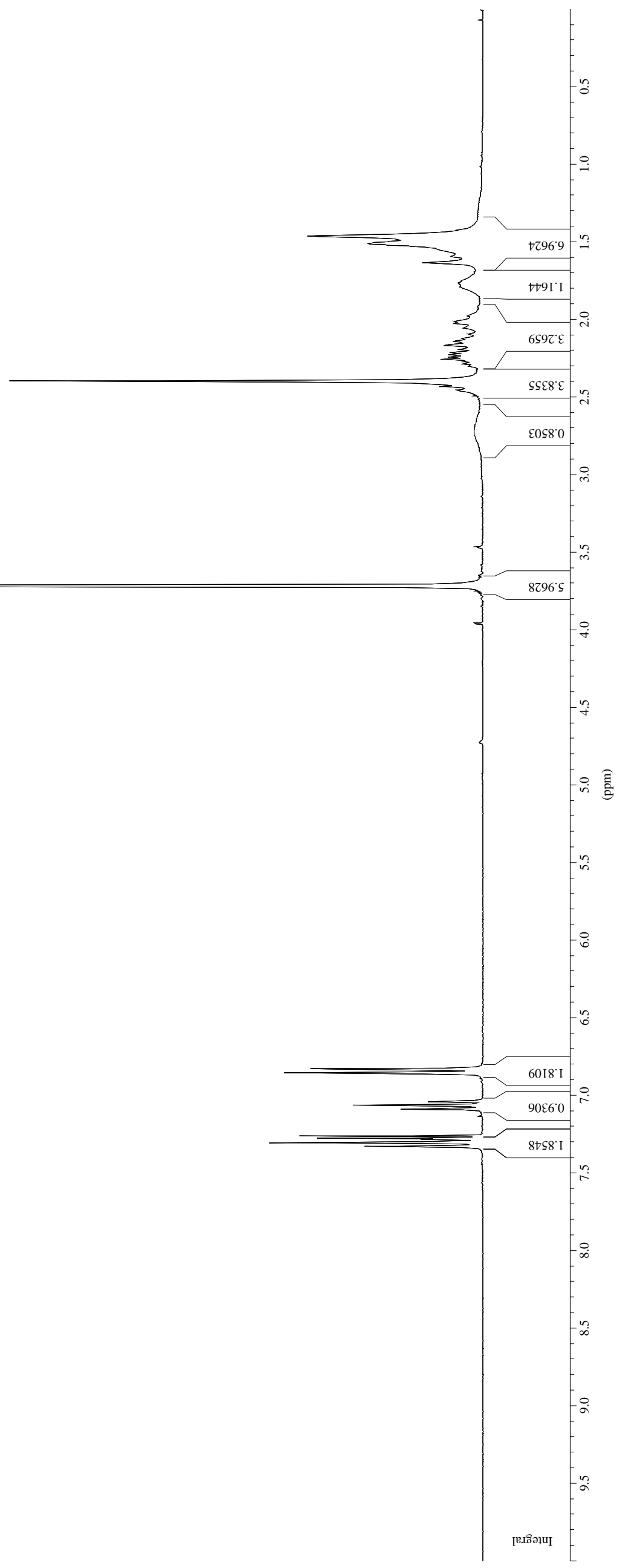


$\varepsilon 0 \angle 0^{\circ} 9 \mathrm{I}$

$\angle \varepsilon 66^{\circ} \mathrm{SZ}$

¿ع06. 92

乙998' $\mathrm{E}$

S006'SE

$902 t \cdot 8 t$

E9t9'zs

IT $\angle 9{ }^{\circ} Z S$

090L'SS

0918.65

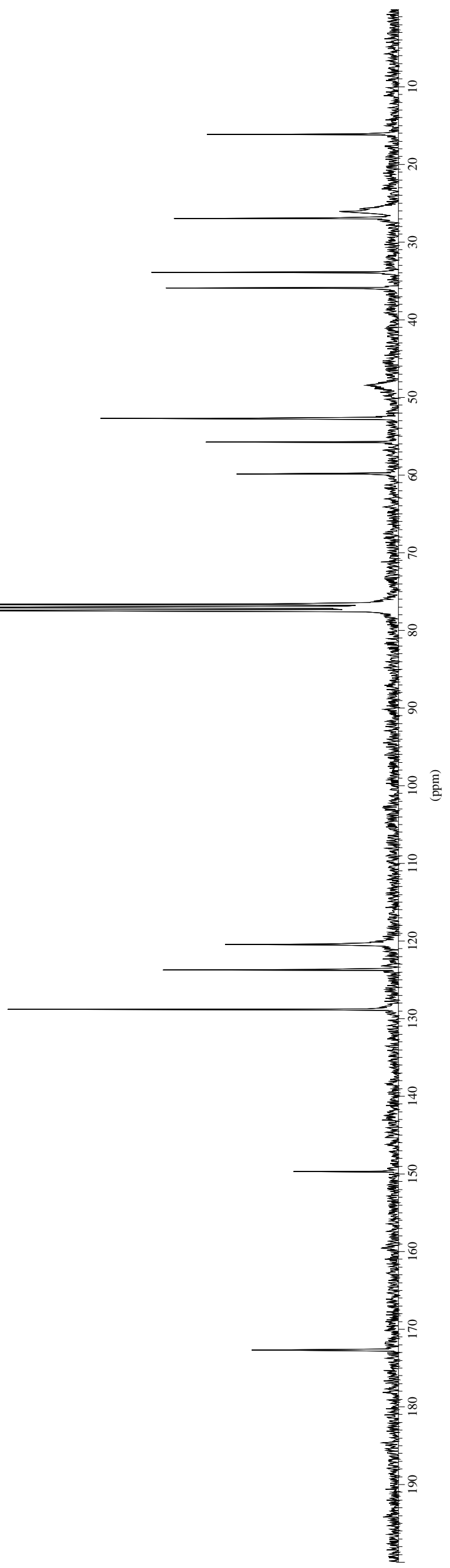

$\varepsilon 8 \angle S^{\prime} 9 L$

$0000 \angle L$

乙EZた゚ 0ZI

IE $\angle 9^{\circ} \varepsilon Z I$

†SLL'8ZI

†6ع9 $6 \mathrm{t} \mathrm{L}$

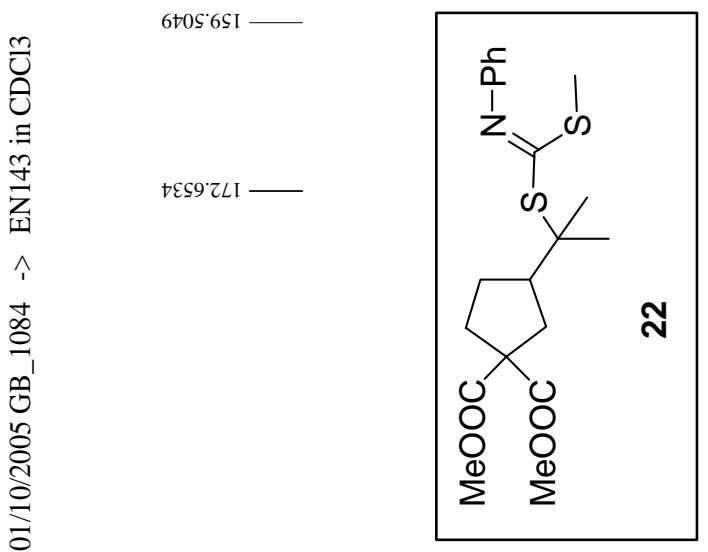




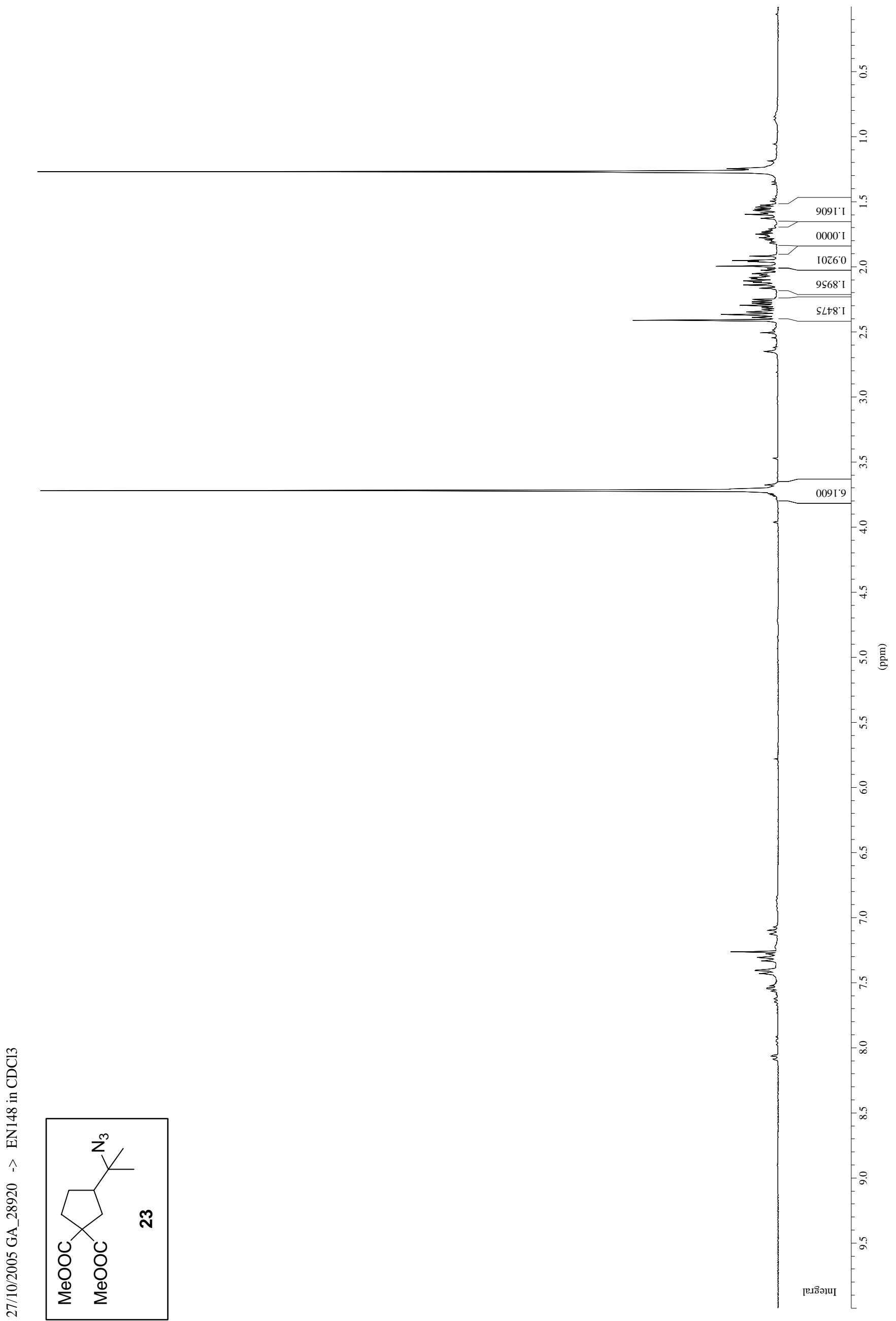


$869 \angle \bullet t$

$\varepsilon \angle 6 S^{\circ} 9 Z$

†979 $๕ \varepsilon$

$9+6 S^{\circ} \mathrm{SE}$

$\angle 8600^{\circ} 6 \mathrm{t}$

ع9t9'zs

9L89 ZS

เ66 ' 6s

689929

E8LS"9L

$0000^{\circ} L L$

8IZt $\angle L$

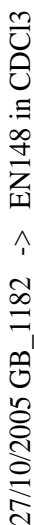

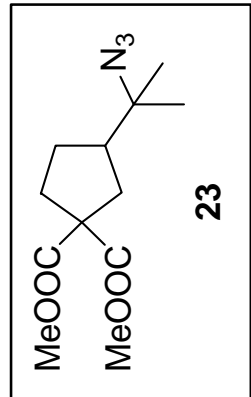




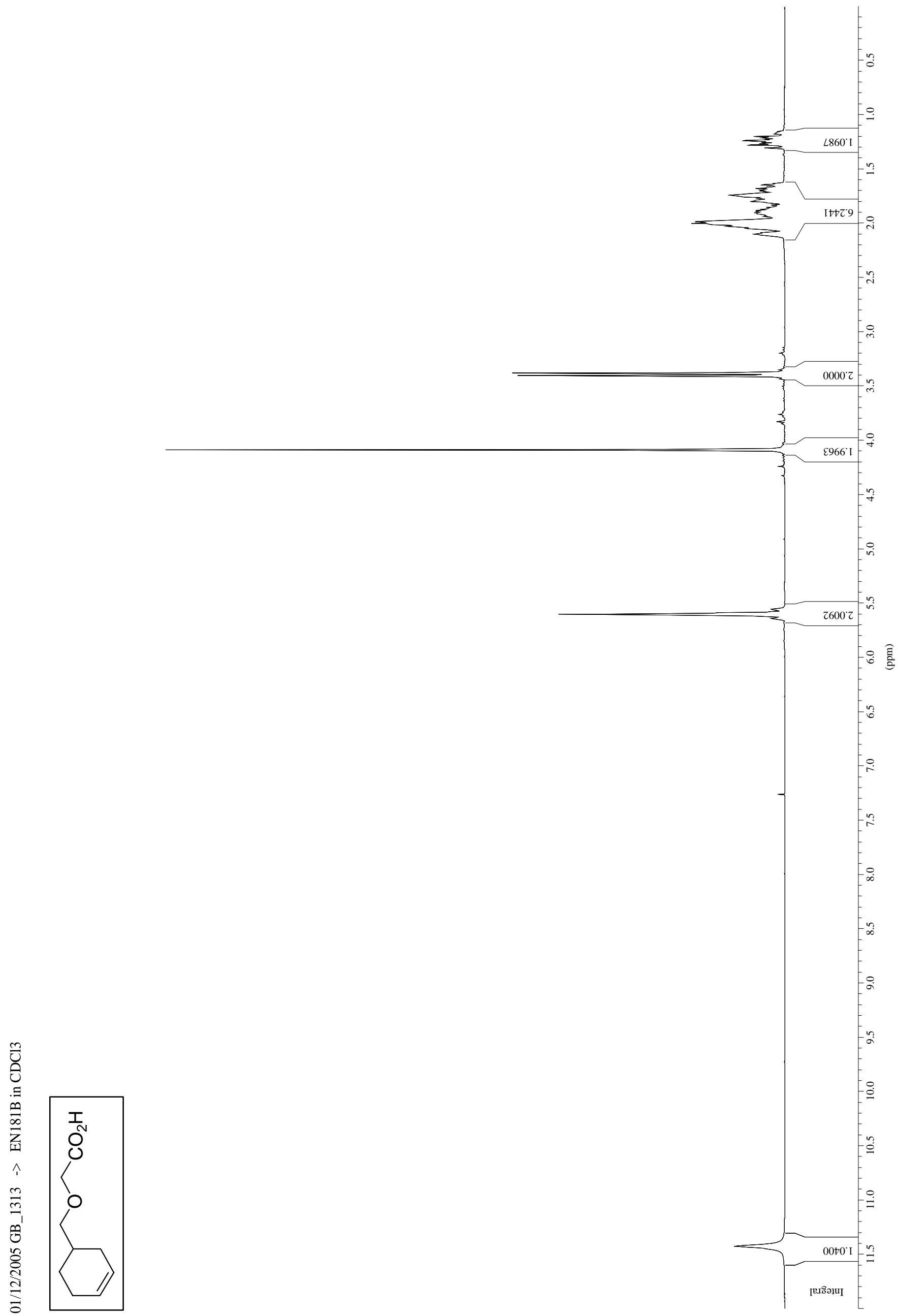


$\angle S I Z \circ Z$

SI6I'SZ

$9 \angle \angle 0.82$

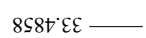

乙8६ L $\angle 9$

IE09.9L

$0000^{\circ} \angle L$

SSZS'SZI

98ฤ8.9ZI

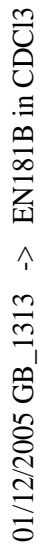

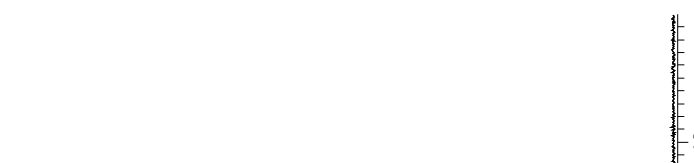




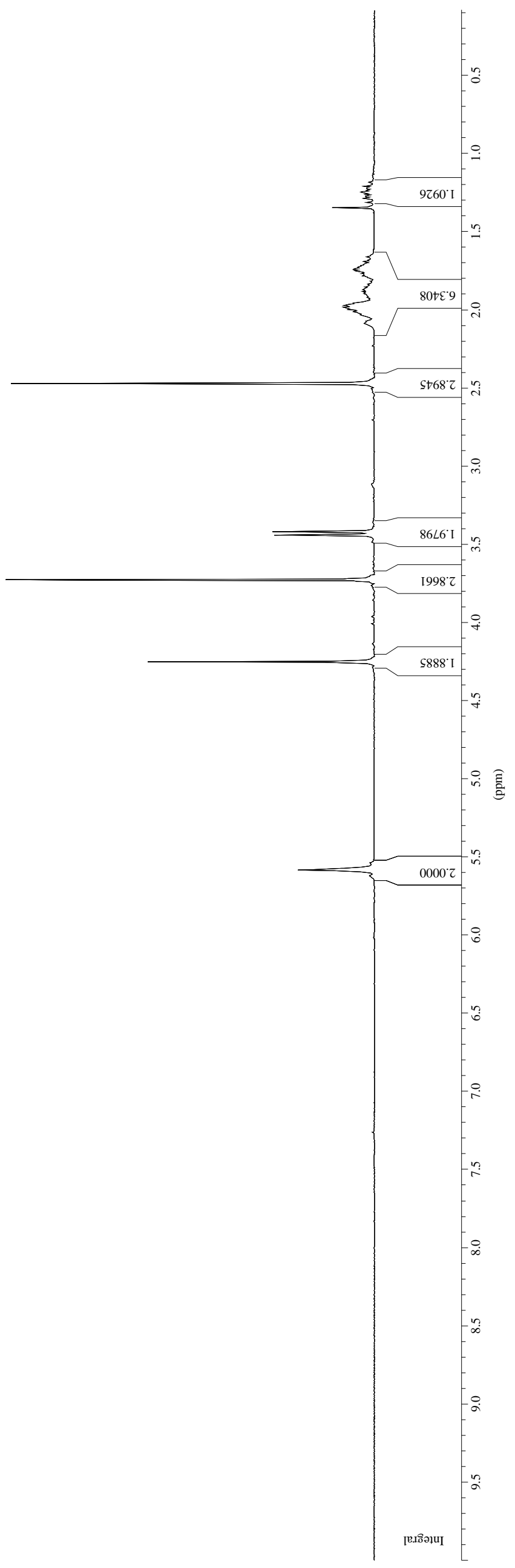


9ZSE $8 \mathrm{I}$

$000 \tau^{\circ} \downarrow \tau$

Eโ00`รZ

I6 $\angle 8 \circ Z$

$858 t^{\circ} \varepsilon \varepsilon$

IZ6E'Zt

0โ6z'99 -

E8LS.9L

$8608^{\circ} 9 L$

$0000^{\circ} \angle L$

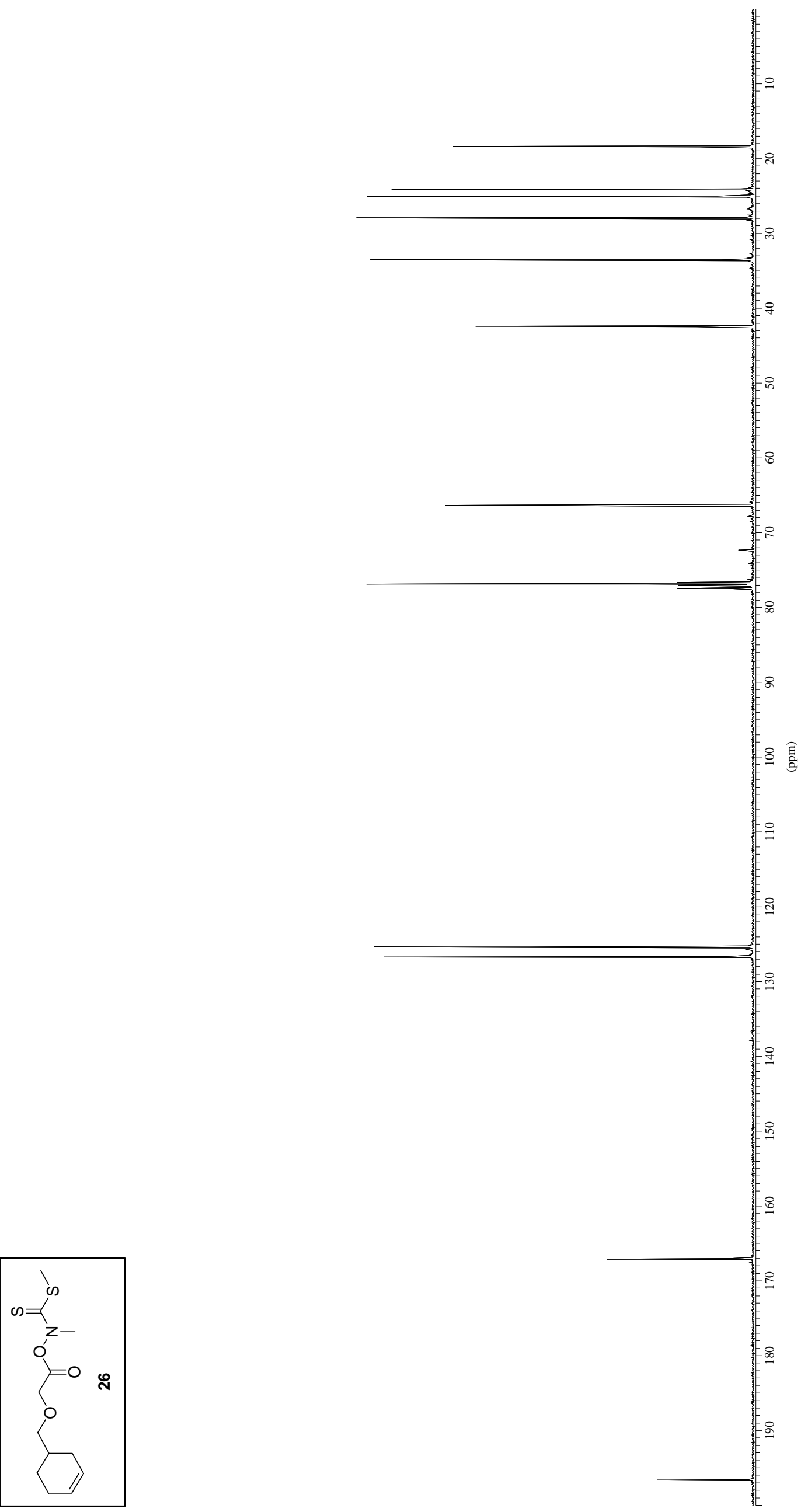

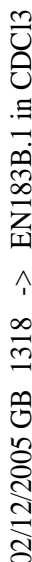

99LE"SZI

$\angle 999^{\circ} 92 \mathrm{~T}$

I0\&0 $\angle 9 \mathrm{I}$

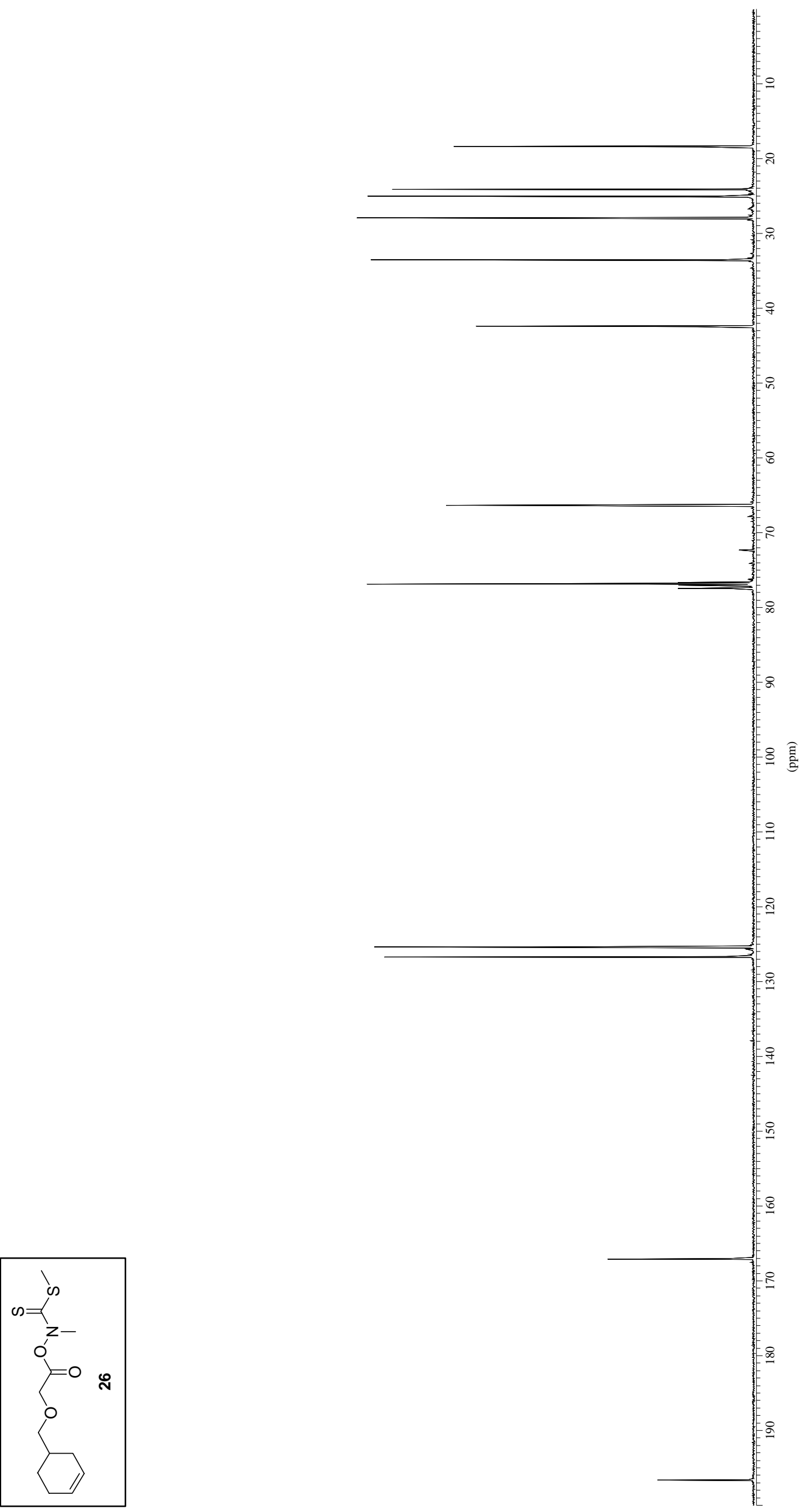

6I09.96I 


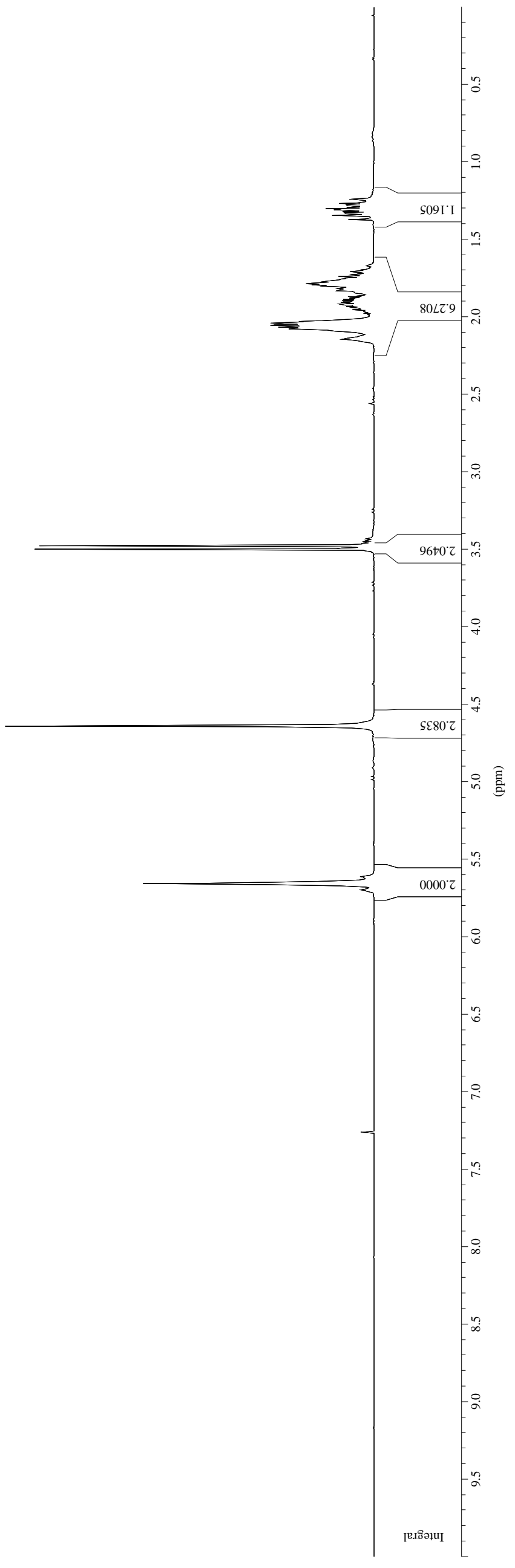


$87<\varepsilon^{\circ}+2$

$\downarrow \varepsilon \angle \varepsilon^{\circ} \varsigma Z$

$0 \varepsilon+z \cdot 8 z$

6Eє $L^{\circ} \varepsilon \mathcal{E}$

$8 \angle L T^{\circ} \forall L$

$00 \angle S^{\circ} 9 L$

$\angle I Z D^{\circ} \angle L$

$90 \angle 6: 28$

$\angle$ ¿Z9'SZI -

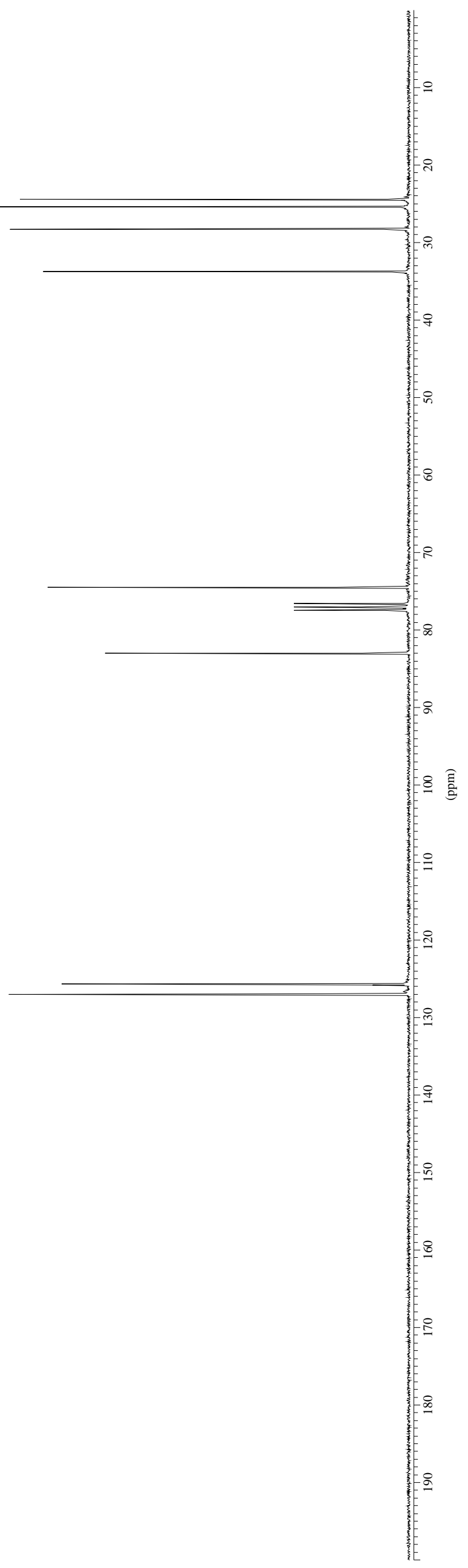

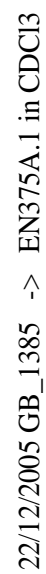

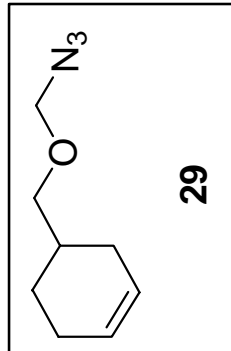

N 